\title{
Description and evaluation of a detailed gas-phase chemistry scheme in the TM5-MP global chemistry transport model (r112)
}

\author{
Stelios Myriokefalitakis $^{1}$, Nikos Daskalakis ${ }^{2}$, Angelos Gkouvousis ${ }^{3,1}$, Andreas Hilboll ${ }^{2, t}$, Twan van Noije ${ }^{4}$, \\ Jason E. Williams ${ }^{4}$, Philippe Le Sager ${ }^{4}$, Vincent Huijnen ${ }^{4}$, Sander Houweling ${ }^{5,6}$, Tommi Bergman ${ }^{7}$, \\ Johann Rasmus Nüß ${ }^{2}$, Mihalis Vrekoussis ${ }^{2,8,9}$, Maria Kanakidou ${ }^{2,3}$, and Maarten C. Krol ${ }^{10,11}$ \\ ${ }^{1}$ Institute for Environmental Research and Sustainable Development (IERSD), \\ National Observatory of Athens, Penteli, Greece \\ ${ }^{2}$ Institute of Environmental Physics, University of Bremen, Bremen, Germany \\ ${ }^{3}$ Environmental Chemical Processes Laboratory (ECPL), Department of Chemistry, University of Crete, Heraklion, Greece \\ ${ }^{4}$ Royal Netherlands Meteorological Institute (KNMI), De Bilt, the Netherlands \\ ${ }^{5}$ Department of Earth Sciences, Vrije Universiteit Amsterdam, the Netherlands \\ ${ }^{6}$ SRON Netherlands Institute for Space Research, Utrecht, the Netherlands \\ ${ }^{7}$ Finnish Meteorological Institute, Climate System Research, Helsinki, Finland \\ ${ }^{8}$ Center for Marine Environmental Sciences, University of Bremen, Bremen, Germany \\ ${ }^{9}$ Energy, Environment and Water Research Center (EEWRC), The Cyprus Institute, Cyprus \\ ${ }^{10}$ Department of Environmental Sciences, Wageningen University, Wageningen, the Netherlands \\ ${ }^{11}$ Institute for Marine and Atmospheric Research (IMAU), Utrecht University, Utrecht, the Netherlands \\ $\boldsymbol{†}_{\text {deceased, } 25 \text { March } 2020}$
}

Correspondence: Stelios Myriokefalitakis (steliosm@noa.gr) and Maarten C. Krol (maarten.krol@wur.nl)

Received: 16 April 2020 - Discussion started: 23 April 2020

Revised: 5 September 2020 - Accepted: 17 September 2020 - Published: 12 November 2020

\begin{abstract}
This work documents and evaluates the tropospheric gas-phase chemical mechanism MOGUNTIA in the three-dimensional chemistry transport model TM5-MP. Compared to the modified CB05 (mCB05) chemical mechanism previously used in the model, MOGUNTIA includes a detailed representation of the light hydrocarbons (C1-C4) and isoprene, along with a simplified chemistry representation of terpenes and aromatics. Another feature implemented in TM5-MP for this work is the use of the Rosenbrock solver in the chemistry code, which can replace the classical Euler backward integration method of the model. Global budgets of ozone $\left(\mathrm{O}_{3}\right)$, carbon monoxide $(\mathrm{CO})$, hydroxyl radicals $(\mathrm{OH})$, nitrogen oxides $\left(\mathrm{NO}_{x}\right)$, and volatile organic compounds (VOCs) are analyzed, and their mixing ratios are compared with a series of surface, aircraft, and satellite observations for the year 2006. Both mechanisms appear to be able to satisfactorily represent observed mixing ratios of important trace gases, with the MOGUNTIA chemistry configuration yielding lower biases than $\mathrm{mCB} 05$ compared to mea-
\end{abstract}

surements in most of the cases. However, the two chemical mechanisms fail to reproduce the observed mixing ratios of light VOCs, indicating insufficient primary emission source strengths, oxidation that is too fast, and/or a low bias in the secondary contribution to $\mathrm{C} 2-\mathrm{C} 3$ organics via VOC atmospheric oxidation. Relative computational memory and time requirements of the different model configurations are also compared and discussed. Overall, the MOGUNTIA scheme simulates a large suite of oxygenated VOCs that are observed in the atmosphere at significant levels. This significantly expands the possible applications of TM5-MP.

\section{Introduction}

Chemistry transport models (CTMs) are tools to effectively study the temporal and spatial evolution of atmospheric species at regional and global scales, as well as to understand how the main physical and chemical processes in the 
troposphere (e.g., emissions, chemistry, transport, and deposition) influence air quality. Model investigations and analyses of the changes in important tropospheric pollutants, such as ozone $\left(\mathrm{O}_{3}\right)$ and carbon monoxide $(\mathrm{CO})$, can further provide essential information about the oxidative capacity of the atmosphere and thus the lifetime of important climate gases like methane $\left(\mathrm{CH}_{4}\right)$. The oxidative capacity also controls the rate of formation and growth of aerosols by conversion of sulfur oxides into particulate sulfate $\left(\mathrm{SO}_{4}^{2-}\right)$ and volatile organic compounds (VOCs) into condensable organic matter that forms organic particles. Under certain tropospheric conditions (e.g., intense sunlight and high temperatures) the oxidation of VOCs in the presence of nitrogen oxides $\left(\mathrm{NO}_{x} \equiv\right.$ $\mathrm{NO}+\mathrm{NO}_{2}$ ) enhances the formation of secondary pollutants, such as $\mathrm{O}_{3}$ (Crutzen, 1974; Derwent et al., 1996; Monks et al., 2009). VOCs and $\mathrm{NO}_{x}$ arise from both natural and anthropogenic emission sources. $\mathrm{NO}_{x}$ can be further converted into other chemical species such as $\mathrm{HNO}_{3}$ and particulate nitrate $\left(\mathrm{NO}_{3}^{-}\right)$that together with $\mathrm{SO}_{4}^{2-}$ are key contributors to atmospheric acidity. The photochemical production of tropospheric $\mathrm{O}_{3}$, a known toxic air pollutant that is transported over long distances, depends on the $\mathrm{NO}_{x}$ and VOC availability in a nonlinear manner (e.g., Seinfeld and Pandis, 2006). Under high- $\mathrm{NO}_{x}$ conditions, common in densely populated areas (i.e., VOC-limited regimes), $\mathrm{O}_{3}$ production is inhibited and reductions in $\mathrm{NO}_{x}$ emissions can locally increase $\mathrm{O}_{3}$. In contrast, in rural areas, $\mathrm{O}_{3}$ production is more efficient, and $\mathrm{NO}_{x}$ emission reductions will decrease $\mathrm{O}_{3}$ (i.e., $\mathrm{NO}_{x}$-limited regimes). Thus, changes in emissions of $\mathrm{NO}_{x}$ and VOC may lead to nonlinear responses in ozone and the oxidation capacity of the troposphere. Overall, understanding the photochemical processes in the troposphere via robust model simulations is key to the development of effective abatement strategies for pollutants that affect both air quality and climate, as well as to the prediction of the future atmospheric composition.

The gas-phase photochemistry in the troposphere consists of numerous and complex reactions between odd oxygen $\left(\mathrm{O}_{x} \equiv \mathrm{O}+\mathrm{O}_{3}\right)$ and $\mathrm{NO}_{x}$, coupled to the oxidation of various VOCs (e.g., Atkinson, 2000; Atkinson et al., 2004). Several chemical mechanisms of varying complexity in the representation of VOC oxidation are currently included in state-of-the-art CTMs. One of the most explicit mechanisms ever built for the simulation of the tropospheric VOC oxidation cycles, the Master Chemical Mechanism (MCM v3), comprises more than 12690 reactions, involving more than 4350 organic species, and about 46 associated inorganic reactions (Jenkin et al., 1997, 2003). Note that recent updates further include detailed aromatic hydrocarbon (Bloss et al., 2005) and isoprene oxidation (Jenkin et al., 2015) mechanisms. Since this level of chemical complexity is far beyond the computational resources potentially available for three-dimensional (3-D) global tropospheric CTMs, simplifications are required that retain the essential features of the chemistry. To this end, various chemical mechanisms of tro- pospheric chemistry have been developed with different levels of complexity, mainly involving reductions of the number of VOCs considered by lumping organic species into representative surrogates. For example, the Statewide Air Pollution Research Center mechanism (SAPRC-99) is a welldocumented gas-phase chemical mechanism used in many CTMs, including a rather detailed representation of tropospheric VOC oxidation based on an evaluation against over 1700 experiments performed in different smog chambers (e.g., Carter, 1995, 2010). SAPRC-99 does not model the oxidation of each VOC individually as the MCM does, but it uses a molecular lumping approach to assign VOCs to a smaller number of reactive species. Other well-documented mechanisms often used in CTMs are the Regional Atmospheric Chemistry Mechanism (RACM; e.g., Geiger et al., 2003; Goliff et al., 2013; Stockwell et al., 1997) and the Model of Ozone and Related Chemical Tracers mechanism (MOZART; Emmons et al., 2010; Horowitz et al., 2003). A molecular lumping mechanism has also been developed and initially used in the Model of the Global Universal Tracer transport In the Atmosphere (MOGUNTIA) 3-D climatological CTM (e.g., Kanakidou and Crutzen, 1999; Poisson et al., 2000; Baboukas et al., 2000) and in box-model applications for field data interpretation (e.g., Poisson et al., 2001; Vrekoussis et al., 2006); the latter chemical mechanism was the starting point for the model development presented here.

A mechanism that has been extensively used in numerous chemistry and climate modeling studies is the Carbon Bond Mechanism (CBM). The CBM has several different versions with different levels of complexity (e.g., reaction rate constant updates, additions of inorganic reactions, and additions of organic species to better represent the respective species and radicals in the atmosphere), such as the CB4 (e.g., Gery et al., 1989; Houweling et al., 1998; Luecken et al., 2008), the CBM 2005 (CB05; e.g., Yarwood et al., 2005; Williams et al., 2013, 2017; Flemming et al., 2015) and the CBM-Z (Zaveri and Peters, 1999). The lumped-structure approach of the CBM has been extensively evaluated against chamber studies (e.g., Yarwood et al., 2005).

Several studies have focused on the impact of the chemical complexity of the gas-phase mechanism on tropospheric simulations. These studies indicate an inevitable compromise between model accuracy and computational efficiency (e.g., Cai et al., 2011; Gross and Stockwell, 2003; Luecken et al., 2008; Sander et al., 2019). Indeed, for a given atmospheric condition, even different versions of the same mechanism (e.g., the CBM family) may give significantly different results. For instance, the more explicit representation of VOCs in CB05 leads to a higher production of $\mathrm{O}_{3}$ compared to the more lumped CB4, mainly due to a higher production of peroxy radicals, aldehydes, and organic peroxides (Saylor and Stein, 2012). A comparison of CB05 with RACM (Kim et al., 2009) revealed that the most considerable differences appeared in areas with significant biogenic emissions due to the more complex chemistry of aldehydes in the presence of 
anthropogenic alkenes and alkanes. Box-model comparisons between the MCM and various state-of-the-art simplified tropospheric chemistry schemes also indicated that the differences between the chemistry schemes can be rather significant under high VOC loadings (Emmerson and Evans, 2009). Thus, the choice of a gas-phase mechanism for a model may introduce uncertainties in predictions of regulated gas-phase pollutants (e.g., Knote et al., 2015). Computational restrictions, such as memory and computing time savings, are always a critical point to consider for large-scale 3-D simulations, especially when higher spatial resolutions are applied. On the other hand, the ability to validate the results of a particular chemical scheme in a global model can be significantly higher for the more extensive schemes that provide an explicit treatment of gases, such as in comparisons with satellite retrievals and in situ observations of a series of individual species.

In this work, a detailed and complete chemistry scheme is implemented in the global CTM TM5-MP, the massively parallel (MP) version of the Tracer Model version 5 (TM5), with the aim to investigate whether the consistent biases in important tropospheric tracers, such as $\mathrm{O}_{3}, \mathrm{CO}, \mathrm{OH}, \mathrm{NO}_{x}$, and light VOCs, found in previous works (e.g., Huijnen et al., 2010; van Noije et al., 2014; Williams et al., 2013, 2017) are sensitive to the chemistry scheme that is used. For this, we use the well-documented tropospheric gas-phase chemistry scheme MOGUNTIA (e.g., Myriokefalitakis et al., 2008, and references therein; along with recent updates) and benchmark its performance in TM5-MP. Section 2 provides a short description of the current model version, focusing on the new features implemented in the gas-phase chemistry and the chemistry integration method. In particular, we describe the implementation of the Kinetic PreProcessor (KPP) software (Damian et al., 2002; Sandu and Sander, 2006) in TM5-MP, which offers higher flexibility for testing, updating, and further developing the chemistry code in the model. Note that we are mostly focusing here on the performance of the new chemical scheme in comparison to the scheme previously included in the model, i.e., the modified CB05 (mCB05). This version of the model was introduced by Huijnen et al. (2010) and Williams et al. (2013) and further updated by Williams et al. (2017). In Sect. 3, the model's performance is analyzed for the different chemical configurations used for this study, and in Sect. 4 a detailed budget analysis of important gas-phase species is presented. Section 5 presents the evaluation of the different configurations of this work. The model's ability to reproduce the variability of important tropospheric species in both space and time is discussed, along with the associated uncertainties in atmospheric burdens and lifetimes. Finally, in Sect. 6 the main conclusions are presented, and some of the benefits and drawbacks of both chemical mechanisms are discussed, together with proposed directions for future model development.

\section{Model description}

\subsection{General}

The well-documented offline 3-D global CTM TM5 (Krol et al., 2005) is used for this study. Historically, the model has evolved from the original TM2 model (Heimann et al., 1988) via the TM3 model (Houweling et al., 1998; Tsigaridis and Kanakidou, 2003) to TM4 (van Noije et al., 2004; Myriokefalitakis et al., 2008) and TM5 (Krol et al., 2005; Huijnen et al., 2010; van Noije et al., 2014; Williams et al., 2017). In TM5-MP, the parallelization of the model has been redesigned, allowing for affordable global simulations at high resolution, i.e., $1^{\circ} \times 1^{\circ}$ globally (Williams et al., 2017). Moreover, in this new MP version, the two-way zoom capability of TM5 is no longer available. All applications of TM5 share the same methods for model discretization and operator splitting (Krol et al., 2005), the treatment of the meteorological fields, and the mass-conserving tracer transport (Bregman et al., 2003). TM5-MP is driven by meteorological fields from the ECMWF ERA-Interim reanalysis (Dee et al., 2011) with an update frequency of $3 \mathrm{~h}$. The advection scheme used is based on the slopes scheme (Russell and Lerner, 1981), and deep and shallow cumulus convection is parameterized according to Tiedtke (1989). The performance of the transport in the model has been evaluated by Peters et al. (2004) using sulfur hexafluoride simulations and by analyzing the vertical and horizontal distribution of radon $\left({ }^{222} \mathrm{Rn}\right)$ (Koffi et al., 2016; Williams et al., 2017). More recently, global transport features, such as the transport times associated with interhemispheric transport, vertical mixing in the troposphere, transport to and in the stratosphere, and transport of air masses between the land and ocean, were evaluated via an intercomparison of six global transport models (Krol et al., 2018).

TM5-MP is primarily designed for simulation of the troposphere (i.e., no explicit stratospheric chemistry is considered in the model). To capture stratospheric ozone effects on actinic fluxes and to ensure realistic ozone stratospheretroposphere exchange (STE), the overhead stratospheric profile is nudged to the ozone dataset provided for the Coupled Model Intercomparison Project phase 6 (CMIP6). The boundary conditions for $\mathrm{CH}_{4}$, both in the lower troposphere and the stratosphere, are also based on the respective global mean value from the CMIP6 dataset (see also Sect. 2.4) to scale the monthly 2-D climatological fields as derived from HALOE measurements (Grooß and Russell, 2005), with the same nudging heights and relaxation times as for the case of stratospheric $\mathrm{O}_{3}$. This approach is justified due to the relatively long lifetime of $\mathrm{CH}_{4}$. Additionally, for $\mathrm{HNO}_{3}$ and $\mathrm{CO}$ in the stratosphere monthly mean latitudinal climatologies derived from ODIN space-based observations are applied by prescribing the ratio of $\mathrm{HNO}_{3} / \mathrm{O}_{3}$ (Jégou et al., 2008; Urban et al., 2009) and $\mathrm{CO} / \mathrm{O}_{3}$ (Dupuy et al., 2004), respectively. Note, however, that when we present the chemical budgets 
in the troposphere, a tropopause definition using the $\mathrm{O}_{3}$ mixing ratio threshold of $150 \mathrm{ppb}$ (e.g., Stevenson et al., 2006) is applied. Moreover, budget results using the $100 \mathrm{ppb} \mathrm{O}_{3}$ mixing ratios (e.g., Lamarque et al., 2012) as a tropopause level in the model are also provided. For clarity, we note that, based on these threshold values, the different model configurations presented in this work (see Sect. 2.5) lead to identical tropopause heights.

The gas-phase chemistry of the TM5-MP model is supplemented with the in-cloud oxidation of $\mathrm{SO}_{2}$ through aqueousphase reactions with $\mathrm{H}_{2} \mathrm{O}_{2}$ and $\mathrm{O}_{3}$ that depend on the acidity of the solution (Dentener and Crutzen, 1993). The heterogeneous conversion of $\mathrm{N}_{2} \mathrm{O}_{5}$ into $\mathrm{HNO}_{3}$ on the available surface area of cloud droplets, cirrus particles, and hydrated sulfate aerosols is also accounted for. For cloud droplets, the number of droplets per unit volume is calculated using the liquid water content provided in the ECMWF meteorological data used by TM5-MP, assuming an effective droplet radius of $8 \mu \mathrm{m}$. For the heterogeneous conversion of $\mathrm{N}_{2} \mathrm{O}_{5}$ on hydrated sulfate particles, the approach of Dentener and Crutzen (1993) is employed using a global mean reaction probability ( $\gamma$ value) of 0.02 and 0.01 on water and ice surfaces, respectively. Heterogeneous conversions also consider the total reactive surface area density of aerosols, with contributions to accumulation-mode aerosol from sulfate, nitrate, and ammonium being calculated by the EQuilibrium Simplified Aerosol Model (EQSAM) approach (Metzger et al., 2002). The distribution of these aerosol species is calculated online and coupled to the gas-phase precursors $\mathrm{NH}_{3}, \mathrm{H}_{2} \mathrm{SO}_{4}$, and $\mathrm{HNO}_{3}$. Note that the aerosol microphysics module M7 (Vignati et al., 2004) is used in the model, as described in Aan de Brugh et al. (2011) and van Noije et al. (2014), along with recent updates on the inclusion of secondary organic aerosols. For $\mathrm{N}_{2} \mathrm{O}_{5}$, the uptake coefficient $(\gamma)$ is considered as a function of temperature and relative humidity (Evans and Jacob, 2005), whilst for $\mathrm{HO}_{2}$ and $\mathrm{NO}_{3}$ radicals fixed $\gamma$ values of 0.06 and $10^{-3}$, respectively, are adopted across all aerosol types (Jacob, 2000).

The model considers the wet removal of atmospheric species by liquid and ice precipitation by both in-cloud and below-cloud scavenging. The fraction of gases removed by precipitation depends on Henry's law (see Table S1 in the Supplement), together with the dissociation constants, temperature, and liquid or ice water content. In-cloud scavenging in stratiform precipitation considers an altitude-dependent precipitation formation rate (also describing the conversion of cloud water into rainwater). For convective precipitation, highly soluble gases are assumed to be scavenged entirely in the vigorous convective updrafts producing rainfall rates of $>1 \mathrm{~mm} \mathrm{~h}^{-1}$. Removal is exponentially scaled down for lower rainfall rates. For the dry deposition, the removal is calculated online in the model based on a series of surface and atmospheric resistances on a $1^{\circ} \times 1^{\circ}$ spatial resolution (Wesely, 1989; Ganzeveld and Lelieveld, 1995; Ganzeveld et al., 1998). Overall, the calculated deposition velocities show both seasonal and diurnal cycles since they are calculated using 3-hourly meteorological and surface parameters based on the uptake resistances for vegetation (in-canopy aerodynamic, soil, and leaf resistance), soil, water, snow, and ice (see Table S2). A more detailed description of dry and wet deposition schemes for the removal of gases can be found in de Bruine et al. (2018).

\subsection{Gas-phase chemistry}

\subsubsection{The original MOGUNTIA chemical scheme}

The new chemical mechanism that has been implemented in TM5-MP for this study was originally developed for box (Poisson et al., 2001) and global (Kanakidou and Crutzen, 1999; Poisson et al., 2000) modeling studies, and it was initially coupled to the global 3-D CTM MOGUNTIA (Zimmermann, 1988). Since then, the scheme has been continuously updated for box modeling, coupled to the global TM4 model, and applied in numerous studies (e.g., Tsigaridis and Kanakidou, 2002; Gros et al., 2002; Myriokefalitakis et al., 2008; Daskalakis et al., 2015).

The MOGUNTIA chemical scheme employs a rather detailed oxidation scheme of light alkanes $\left(\mathrm{CH}_{4}, \mathrm{C}_{2} \mathrm{H}_{6}\right.$, and $\left.\mathrm{C}_{3} \mathrm{H}_{8}\right)$, light alkenes $\left(\mathrm{C}_{2} \mathrm{H}_{4}\right.$ and $\left.\mathrm{C}_{3} \mathrm{H}_{6}\right)$, acetylene $\left(\mathrm{C}_{2} \mathrm{H}_{2}\right)$, and isoprene $\left(\mathrm{C}_{5} \mathrm{H}_{8}\right)$. Acetaldehyde $\left(\mathrm{CH}_{3} \mathrm{CHO}\right)$, glyoxal (GLY; CHOCHO), glycolaldehyde (GLYAL; $\mathrm{HOCH}_{2} \mathrm{CHO}$ ), methylglyoxal (MGLY; $\mathrm{CH}_{3} \mathrm{C}(\mathrm{O}) \mathrm{CHO}$ ), and acetone $\left(\mathrm{CH}_{3} \mathrm{COCH}_{3}\right)$ are also explicitly treated in the mechanism. The oxidation pathways of methacrolein (MACR; $\mathrm{CH}_{3}\left(\mathrm{CH}_{2}\right) \mathrm{CH}=\mathrm{O}$ ) and methylvinyl ketone (MVK; $\left.\mathrm{CH}_{3} \mathrm{C}(\mathrm{O}) \mathrm{CH}=\mathrm{CH}_{2}\right)$ are also considered, together with the formation of formic $(\mathrm{HCOOH})$ and acetic acid $\left(\mathrm{CH}_{3} \mathrm{COOH}\right)$. Higher VOCs (i.e., $\mathrm{C}_{n>4}$ ), besides isoprene, are represented in the mechanism by the surrogate species $n$-butane $(n$ $\mathrm{C}_{4} \mathrm{H}_{10}$ ), motivated by the similar $\mathrm{O}_{x}$ and hydrogen oxides $\left(\mathrm{HO}_{x}\right)$ yields per oxidized carbon atom (see, e.g., Poisson et al., 2000; Stavrakou et al., 2009a). The second-generation oxidation products of higher hydrocarbons of biogenic origin (such as terpenes) and aromatics are also considered to follow the gas-phase oxidation pathways of the respective isoprene and surrogate $n-\mathrm{C}_{4} \mathrm{H}_{10}$ oxidation species.

The reactions of peroxy radicals $\left(\mathrm{RO}_{2}\right)$ with hydrogen peroxide $\left(\mathrm{HO}_{2}\right)$, methyl peroxide $\left(\mathrm{CH}_{3} \mathrm{O}_{2}\right)$, and $\mathrm{NO}$ lead to organic hydroperoxides $(\mathrm{ROOH})$, carbonyls, and organic nitrates, respectively. $\mathrm{ROOH}$ is removed by photolysis and reaction with $\mathrm{OH}$. The addition of $\mathrm{NO}$ to the formed $\mathrm{RO}_{2}$ radicals leads to alkyl nitrates $\left(\mathrm{RONO}_{2}\right)$, which are much longer-lived than $\mathrm{NO}_{x} \cdot \mathrm{RONO}_{2}$ can thus be transported over longer distances than $\mathrm{NO}_{x}$ and serve as a sink for $\mathrm{NO}_{x}$ in high- $\mathrm{NO}_{x}$ regimes and as a source for $\mathrm{NO}_{x}$ in low- $\mathrm{NO}_{x}$ regimes. The $\mathrm{RONO}_{2}$ compounds explicitly considered in this study are identified by $\mathrm{R}=\mathrm{CH}_{3}, \mathrm{C}_{2} \mathrm{H}_{5}, \mathrm{C}_{3} \mathrm{H}_{7}, \mathrm{C}_{4} \mathrm{H}_{9}$, $\mathrm{HOC}_{2} \mathrm{H}_{4} \mathrm{O}$, and $\mathrm{C}_{5} \mathrm{H}_{8}(\mathrm{OH})$, i.e., the first-generation product of isoprene oxidation. Additionally, the reactions of the 
acyl peroxy radicals $\left(\mathrm{RC}(\mathrm{O}) \mathrm{O}_{2}\right)$ with $\mathrm{NO}_{2}$ produce peroxyacyl nitrates $\left(\mathrm{RC}(\mathrm{O}) \mathrm{O}_{2} \mathrm{NO}_{2}\right)$, in particular peroxyacetyl nitrate $\left(\mathrm{PAN} ; \mathrm{R}=\mathrm{CH}_{3}\right.$ ), which is the most abundant organic nitrate observed in the troposphere and the only species of this group that is considered here. Thermal decomposition is dominant for peroxyacyl nitrates, while it is negligible for alkyl nitrates. $\mathrm{NO}_{3}$ radical reactions with aldehydes, alcohols, $n-\mathrm{C}_{4} \mathrm{H}_{10}$, dimethylsulfide (DMS), and unsaturated hydrocarbons are also considered. A more detailed description of the chemical scheme used for this study can be found in Poisson et al. (2000) and Myriokefalitakis et al. (2008).

\subsubsection{Updates of the MOGUNTIA chemical mechanism}

Several updates have been applied to the original MOGUNTIA chemical scheme with respect to the previous implementations (e.g., Poisson et al., 2000; Myriokefalitakis et al., 2008). These updates include reactions of major hydrocarbons, their rate constants, and oxidation pathways. Concerning the terpene chemistry, we consider one lumped monoterpene species $\left(\mathrm{C}_{10} \mathrm{H}_{16}\right)$ for all terpenes (assuming a 50:50 $\alpha-: \beta$-pinene distribution), in contrast to the consideration of the explicit oxidation of $\alpha$ - and $\beta$-pinene as performed in the previous implementations of the MOGUNTIA scheme (e.g., Myriokefalitakis et al., 2008, 2010). Thus, monoterpenes represent all terpenes and terpenoid species here. Likewise, toluene is used to represent all aromatics replacing benzene, xylene, and toluene used previously (Myriokefalitakis et al., 2008, 2010). Besides these compounds, toluene is also used to represent trimethyl-benzenes and higher aromatics. Moreover, for this work the coupling of the gas-phase chemistry with the aqueous-phase oxidation scheme of $\mathrm{SO}_{2}$, as well as the gas-phase oxidation of dimethyl sulfide (DMS), methyl sulfonic acid (MSA), and ammonia $\left(\mathrm{NH}_{3}\right)$, follows the oxidation scheme outlined by Williams et al. (2013), which is slightly simpler compared to the MOGUNTIA scheme used in previous studies (e.g., Myriokefalitakis et al., 2010). Note that the lumping mentioned above, and the simplifications implemented here, aim at limiting the number of species without degrading the general performance of the chemical scheme for global-scale tropospheric chemistry.

Isoprene (2-methyl-1,3-butadiene; ISOP) oxidation has been extended with the production of isoprene epoxydiols (IEPOXs) and hydroperoxyaldehydes (HPALDs), as well as the $\mathrm{HO}_{x}$ recycling mechanism under low- $\mathrm{NO}_{x}$ conditions (Paulot et al., 2009; Peeters and Müller, 2010; Crounse et al., 2011; Browne et al., 2014). The latter species replaces the lumped second-generation oxidation product considered in previous implementations of the MOGUNTIA mechanism (Poisson et al., 2000; Myriokefalitakis et al., 2008). The oxidation of isoprene by the $\mathrm{OH}$ radical leads to the formation of several isomers of an unsaturated hydroxy hydroperoxide. In the presence of $\mathrm{NO}_{x}$, this leads to the formation of carbonyl compounds. However, under low- $\mathrm{NO}_{x}$ conditions, the major product from unsaturated hydroxy hydroperox- ide oxidation is IEPOX (i.e., cis- and trans-isomers). The organic peroxy radicals formed from $\mathrm{OH}$ oxidation of isoprene can react with either (1) $\mathrm{HO}_{2}$ to form hydroperoxides or (2) NO to form hydroxynitrates, formaldehyde (HCHO), MVK, MACR and $\mathrm{HO}_{2}$ (e.g., Paulot et al., 2009), or hydroperoxyenals (HPALDs). The latter are produced by the isomerization of the initial isoprene organic hydroperoxy radicals followed by reaction with $\mathrm{O}_{2}$ and other oxidized products (Peeters et al., 2009; Peeters and Müller, 2010). Under $\mathrm{HO}_{2}$-dominated conditions, the main products are unsaturated hydroperoxides (all possible isomers referred to as ISOPOOH; see Table 2). The fate of isoprene peroxy radicals is highly dependent on the mixing ratios of $\mathrm{HO}_{2}, \mathrm{NO}$, organic peroxy radicals, and the local meteorological conditions that affect thermal and photochemical reaction rates and wet and dry removal. Subsequent reactions of ISOPOOH with $\mathrm{OH}$ produce epoxydiols ( $c i s$ - and trans-isomers referred to as IEPOXs) and regenerate OH radicals (Paulot et al., 2009). Moreover, the isoprene peroxy radical 1,6-H-shift isomerizations (Peeters et al., 2014; Peeters and Müller, 2010) lead to the formation of photolabile C5-hydroperoxyaldehydes (i.e., all possible isomers referred to as HPALDs; see Table 1). Overall, these additions to the chemistry scheme are expected to provide a better representation of $\mathrm{OH}$ regeneration during isoprene oxidation (e.g., Browne et al., 2014) compared to the previous implementation of the MOGUNTIA mechanism.

The MOGUNTIA chemistry scheme is in line with the VOC oxidation pathways as proposed by the Master Chemistry Mechanism (MCM v3.3.1) (e.g., Bloss et al., 2005; Saunders et al., 2003). The thermal and pressure-dependent reaction rate coefficients of the MOGUNTIA chemical mechanism are taken (when available) from the IUPAC kinetic data evaluation (Atkinson et al., 2004; Wallington et al., 2018) and supplemented with reaction rates based on recommendations given by the JPL (Burkholder et al., 2015). Photolysis frequencies needed to drive MOGUNTIA are taken from the IUPAC database (Atkinson, 1997; Atkinson et al., 2004) along with the updates from MCM v3.3.1 (Bloss et al., 2005; Jenkin et al., 1997, 2003, 2015; Saunders et al., 2003). Note that the model calculates the photolysis frequencies online as described in Williams et al. (2012). Comprehensive lists of all photochemical and thermal kinetic reactions included in the current MOGUNTIA chemical scheme are presented in Tables 1 and 2, respectively.

\subsection{The chemical solver}

The KPP version 2.2.3 (Damian et al., 2002; Sandu and Sander, 2006) is employed here to generate Fortran 90 code for the numerical integration of the gas-phase chemical mechanisms. An important advantage of this approach is that the implementation of a KPP-generated code in the model is less prone to errors than coding the mechanism manually. Upon the translation of the chemistry mechanisms (e.g., 
Table 1. Photolysis reactions (J) in the MOGUNTIA chemistry scheme.

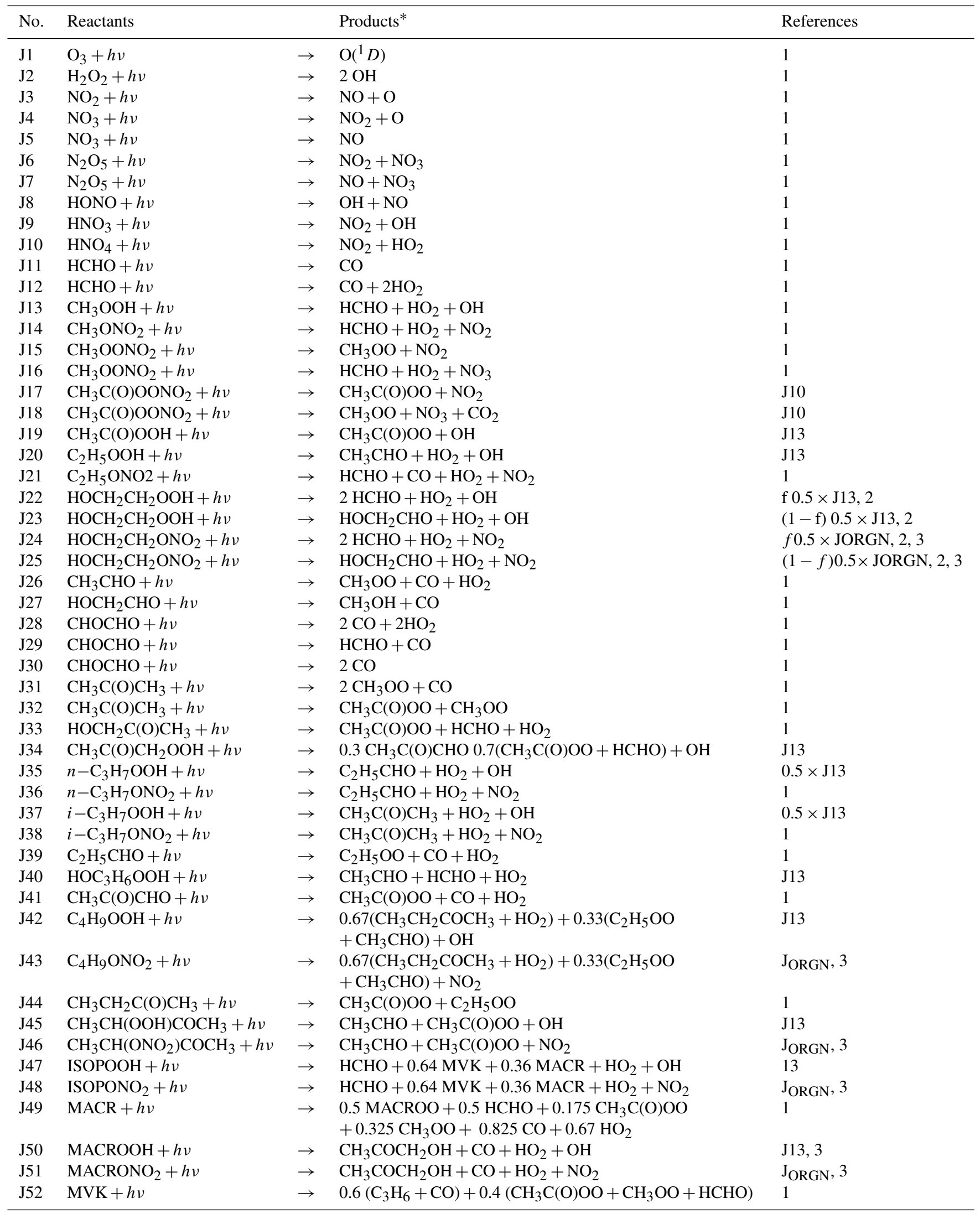


Table 1. Continued.

\begin{tabular}{|c|c|c|c|c|}
\hline No. & Reactants & & Products* & References \\
\hline $\mathrm{J} 53$ & $\mathrm{MVKOOH}+h v$ & $\rightarrow$ & $\begin{array}{l}0.7\left(\mathrm{CH}_{3} \mathrm{C}(\mathrm{O}) \mathrm{OO}+\mathrm{HOCH}_{2} \mathrm{CHO}\right)+0.3\left(\mathrm{CH}_{3} \mathrm{C}(\mathrm{O}) \mathrm{CHO}\right. \\
\left.+\mathrm{HCHO}+\mathrm{HO}_{2}\right)+\mathrm{OH}\end{array}$ & J13 \\
\hline $\mathrm{J} 54$ & $\mathrm{MVKONO}_{2}+h v$ & $\rightarrow$ & $\begin{array}{l}0.7\left(\mathrm{CH}_{3} \mathrm{C}(\mathrm{O}) \mathrm{OO}+\mathrm{HOCH}_{2} \mathrm{CHO}\right)+0.3\left(\mathrm{CH}_{3} \mathrm{C}(\mathrm{O}) \mathrm{CHO}\right. \\
\left.+\mathrm{HCHO}+\mathrm{HO}_{2}\right)+\mathrm{NO}_{2}\end{array}$ & $\mathrm{~J}_{\mathrm{ORGN}}, 3$ \\
\hline $\mathrm{J} 55$ & $\mathrm{CH}_{3} \mathrm{C}(\mathrm{O}) \mathrm{C}(\mathrm{O}) \mathrm{CH}_{3}+h v$ & $\rightarrow$ & $2 \mathrm{CH}_{3} \mathrm{C}(\mathrm{O}) \mathrm{OO}$ & 1 \\
\hline J56 & $\mathrm{CH}_{3} \mathrm{C}(\mathrm{O}) \mathrm{COOH}+h v$ & $\rightarrow$ & $\mathrm{CH}_{3} \mathrm{C}(\mathrm{O}) \mathrm{OO}+\mathrm{HO}_{2}+\mathrm{CO}_{2}$ & 1 \\
\hline J57 & HPALD $+h v$ & $\rightarrow$ & $\begin{array}{l}0.5 \quad \mathrm{HOCH}_{2} \mathrm{C}(\mathrm{O}) \mathrm{CH}_{3}+0.5 \quad \mathrm{CH}_{3} \mathrm{C}(\mathrm{O}) \mathrm{CHO}+0.25 \\
\mathrm{HOCH}_{2} \mathrm{CHO} \\
+0.25 \mathrm{CHOCHO}+\mathrm{HCHO}+\mathrm{HO}_{2}+\mathrm{OH}\end{array}$ & 4,5 \\
\hline $\mathrm{J} 58$ & $\mathrm{O}_{2}+h v$ & $\rightarrow$ & $\mathrm{O}_{3}$ & 1 \\
\hline
\end{tabular}

* The reaction products $\mathrm{O}_{2}, \mathrm{H}_{2}$, and $\mathrm{H}_{2} \mathrm{O}$ are not shown. ${ }^{1}$ http://iupac.pole-ether.fr (last access: 20 August 2019)

2 Atkinson (1997): $R_{1}=2.7 \times 10^{14} \exp (-6350 / T) ; R_{2}=6.3 \times 10^{-14} \exp (-550 / T) ; f=R_{1} /\left(R_{1}+R_{2} \times\left[\mathrm{O}_{2}\right]\right)$.

${ }^{3} \mathrm{~J}_{\mathrm{ORGN}}$ is calculated based on average $\sigma$ values for $1-\mathrm{C}_{4} \mathrm{H}_{9} \mathrm{ONO}_{2}$ and $2-\mathrm{C}_{4} \mathrm{H}_{9} \mathrm{ONO}_{2}$ as described in Williams et al. (2012).

${ }^{4}$ Browne et al. (2014). ${ }^{5}$ Peeters and Müller (2010).

species, reactions, rate coefficients) from the KPP language into a Fortran 90 code, a model driver was developed to arrange the respective couplings to TM5-MP. Minor changes, however, were needed in the KPP code to deal with TM5MP I/O requirements. The photolysis and thermal reactions are not calculated in KPP but explicitly calculated by the respective modules of TM5-MP and then directly provided to the aforementioned chemistry driver. To this end, only the integration method has been updated in the model, replacing the default hand-coded chemical solver setup. Moreover, the NO emission rates (and the dry deposition terms of all deposited species) are imported to KPP through the application of appropriate production (and loss) rates, as previously done for the Euler backward iterative (EBI) solver, owing mainly to the numerical stiffness of the $\mathrm{NO}-\mathrm{NO}_{2}-\mathrm{O}_{3}$ photostationary state and their fast interactions (see, e.g., Huijnen et al., 2010). In this study, the Rosenbrock solver is used as the numerical integrator (Sander et al., 2019). The Rosenbrock solver has been shown to be robust and capable of integrating very stiff sets of equations (Sander et al., 2011). For all previous versions of the model, the EBI solver (Hertel et al., 1993) was used. This holds for the modified CB4 (Houweling et al., 1998), mCB05 (Williams et al., 2013), and MOGUNTIA (Myriokefalitakis et al., 2008) mechanisms. Note, however, that EBI was originally designed for the CB4 mechanism (Gery et al., 1989), and it is a rather fast and robust solver suitable for use in large-scale atmospheric models that incorporate operator splitting (Huang and Chang, 2001).

The favorable comparison of the Rosenbrock solver against other widely used methods, such as FACSIMILE (Curtis and Sweetenham, 1987), has already been described in the literature (e.g., Sander et al., 2005). Focusing specifically on the comparison of a series of Rosenbrock solvers to EBI, Sandu et al. (1997) concluded that, although EBI appears robust, especially when it is used with a relatively large time step, the Rosenbrock methods with variable time steps are significantly more accurate and clearly superior for accuracies in the range of $1 \%$ compared to EBI for a range of species examined. The main aim of this study is not to compare the two chemistry solvers (i.e., the Rosenbrock vs. the EBI). Instead, we present model simulations using the Rosenbrock solver as produced by KPP for the mCB05 scheme (see Sect. 2.5) to isolate the impact of the solver on various species mixing ratios in this work.

\subsection{Emission setup}

For the present study, emissions from anthropogenic activities, including aircraft emissions (Hoesly et al., 2018) and biomass burning (speciated for agricultural waste burning, deforestation fires, boreal forest fires, peat fires, savanna fires, and temperate forest fires; van Marle et al., 2017), are adopted from the sectoral and gridded historical inventories as developed for CMIP6 (Eyring et al., 2016). In more detail, anthropogenic and biomass burning emissions of $\mathrm{CO}, \mathrm{NO}_{x}$, black carbon aerosol (BC), particulate organic carbon (OC), sulfur dioxide and sulfates $\left(\mathrm{SO}_{x}\right)$, and speciated non-methane volatile organic compounds (NMVOCs) are considered, such as emissions of ethane $\left(\mathrm{C}_{2} \mathrm{H}_{6}\right)$, methanol $\left(\mathrm{CH}_{3} \mathrm{OH}\right)$, ethanol $\left(\mathrm{C}_{2} \mathrm{H}_{5} \mathrm{OH}\right)$, propane $\left(\mathrm{C}_{3} \mathrm{H}_{8}\right)$, acetylene $\left(\mathrm{C}_{2} \mathrm{H}_{2}\right)$, ethane $\left(\mathrm{C}_{2} \mathrm{H}_{4}\right)$, propene $\left(\mathrm{C}_{3} \mathrm{H}_{6}\right)$, isoprene $\left(\mathrm{C}_{5} \mathrm{H}_{8}\right)$, monoterpenes $\left(\mathrm{C}_{10} \mathrm{H}_{16}\right)$, benzene $\left(\mathrm{C}_{6} \mathrm{H}_{6}\right)$, toluene $\left(\mathrm{C}_{7} \mathrm{H}_{8}\right)$, xylene $\left(\mathrm{C}_{8} \mathrm{H}_{10}\right)$ and other aromatics, higher alkenes, higher alkanes, $\mathrm{HCHO}$, acetaldehyde $\left(\mathrm{CH}_{3} \mathrm{CHO}\right)$, acetone $\left(\mathrm{CH}_{3} \mathrm{COCH}_{3}\right)$, dimethylsulfide (DMS; $\left.\mathrm{C}_{2} \mathrm{H}_{6} \mathrm{~S}\right)$, formic acid $(\mathrm{HCOOH})$, acetic acid $\left(\mathrm{CH}_{3} \mathrm{COOH}\right)$, methyl ethyl ketone (MEK; $\mathrm{CH}_{3} \mathrm{CH}_{2} \mathrm{COCH}_{3}$ ), methylglyoxal (MGLY; $\mathrm{CH}_{3} \mathrm{C}(\mathrm{O}) \mathrm{CHO}$ ), and hydroxyacetaldehyde $\left(\mathrm{HOCH}_{2} \mathrm{CHO}\right)$. Note that all biomass burning emissions (open forest and grassland fires) are vertically distributed in the model over latitude-dependent injection heights, i.e., for tropical $\left(30^{\circ} \mathrm{S}-30^{\circ} \mathrm{N}\right)$, temperate $\left(30-60^{\circ} \mathrm{S}-\mathrm{N}\right)$, and high- 
Table 2. Thermal reactions (K) in the MOGUNTIA chemistry scheme.

\begin{tabular}{|c|c|c|c|c|c|}
\hline No. & Reactants & & Products* & Rate expression & References \\
\hline K0a & $\mathrm{O}\left({ }^{1} \mathrm{D}\right)(+\mathrm{M})$ & $\rightarrow$ & $\mathrm{O}$ & $\begin{array}{l}3.3 \times 10^{-11} \exp (55 / T)\left[\mathrm{O}_{2}\right]+2.5 \times \\
10^{-11} \exp (110 / T)\left[\mathrm{N}_{2}\right]\end{array}$ & 1 \\
\hline KOb & $\mathrm{O}\left({ }^{1} \mathrm{D}\right)+\mathrm{H}_{2} \mathrm{O}$ & $\rightarrow$ & $\mathrm{OH}+\mathrm{OH}$ & $1.63 \times 10^{-10} \exp (60 / T)$ & 1 \\
\hline K1 & $\mathrm{O}_{3}+\mathrm{OH}$ & $\rightarrow$ & $\mathrm{HO}_{2}$ & $1.7 \times 10^{-12} \exp (-940 / T)$ & 1 \\
\hline $\mathrm{K} 2$ & $\mathrm{HO}_{2}+\mathrm{O}_{3}$ & $\rightarrow$ & $\mathrm{OH}^{-}$ & $2.03 \times 10^{-16}(T / 300)^{4.57} \exp (693 / T)$ & 1 \\
\hline K3 & $\mathrm{HO}_{2}+\mathrm{OH}$ & $\rightarrow$ & $\mathrm{H}_{2} \mathrm{O}$ & $4.8 \times 10^{-11} \exp (250 / T)$ & 1 \\
\hline K4 & $\mathrm{HO}_{2}+\mathrm{HO}_{2}$ & $\rightarrow$ & $\mathrm{H}_{2} \mathrm{O}_{2}$ & $\begin{array}{l}2.2 \times 10^{-13} \exp (600 / T) \\
1.9 \times 10^{-33}\left[\mathrm{~N}_{2}\right] \exp (980 / T) \\
1.4 \times 10^{-21}\left[\mathrm{H}_{2} \mathrm{O}\right] \exp (2200 / T)\end{array}$ & 1 \\
\hline K5 & $\mathrm{H}_{2} \mathrm{O}_{2}+\mathrm{OH}$ & $\rightarrow$ & $\mathrm{HO}_{2}$ & $2.9 \times 10^{-12} \exp (-160 / T)$ & 1 \\
\hline K6 & $\mathrm{HO}_{2}+\mathrm{NO}$ & $\rightarrow$ & $\mathrm{NO}_{2}+\mathrm{HO}$ & $3.45 \times 10^{-12} \exp (270 / T)$ & 1 \\
\hline K7 & $\mathrm{NO}+\mathrm{O}_{3}$ & $\rightarrow$ & $\mathrm{NO}_{2}$ & $2.07 \times 10^{-12} \exp (-1400 / T)$ & 1 \\
\hline K8 & $\mathrm{NO}+\mathrm{NO}_{3}$ & $\rightarrow$ & $2 \mathrm{NO}_{2}$ & $1.8 \times 10^{-11} \exp (110 / T)$ & 1 \\
\hline K9 & $\mathrm{NO}_{2}+\mathrm{O}_{3}$ & $\rightarrow$ & $\mathrm{NO}_{3}$ & $1.4 \times 10^{-13} \exp (-2470 / T)$ & 1 \\
\hline K10 & $\mathrm{OH}+\mathrm{NO}\{+\mathrm{M}\}$ & $\rightarrow$ & HONO & $\begin{array}{l}7.4 \times 10^{-31} \times(T / 300)^{-2.4}\left[\mathrm{~N}_{2}\right] \\
3.3 \times 10^{-11}(T / 300)^{-0.3} \\
\mathrm{Fc}=0.81\end{array}$ & 1 \\
\hline K11 & $\mathrm{OH}+\mathrm{NO}_{2}\{+\mathrm{M}\}$ & $\rightarrow$ & $\mathrm{HONO}_{2}$ & $\begin{array}{l}3.2 \times 10^{-30}(T / 300)^{-4.5}\left[\mathrm{~N}_{2}\right] \\
3.0 \times 10^{-11} \\
\mathrm{Fc}=0.41\end{array}$ & 1 \\
\hline K12 & $\mathrm{NO}_{2}+\mathrm{NO}_{3}\{+\mathrm{M}\}$ & $\rightarrow$ & $\mathrm{N}_{2} \mathrm{O}_{5}$ & $\begin{array}{l}3.6 \times 10^{-30}(T / 300)^{-4.1}\left[\mathrm{~N}_{2}\right] \\
1.9 \times 10^{-12}(T / 300)^{0.2} \\
\mathrm{Fc}=0.35\end{array}$ & 1 \\
\hline K13 & $\mathrm{NO}_{2}+\mathrm{HO}_{2}$ & $\rightarrow$ & $\mathrm{HO}_{2} \mathrm{NO}_{2}$ & $\begin{array}{l}1.4 \times 10^{-31}(T / 300)^{-3.1}\left[\mathrm{~N}_{2}\right] \\
4.0 \times 10^{-12} \\
\mathrm{Fc}=0.40\end{array}$ & 1 \\
\hline K14 & $\mathrm{HO}_{2}+\mathrm{NO}_{3}$ & $\rightarrow$ & $\mathrm{HNO}_{3}$ & $4.0 \times 10^{-12}$ & 1 \\
\hline K15 & $\mathrm{HONO}+\mathrm{OH}$ & $\rightarrow$ & $\mathrm{NO}_{2}$ & $2.5 \times 10^{-12} \exp (260 / T)$ & 1 \\
\hline K16 & $\mathrm{HNO}_{3}+\mathrm{OH}$ & $\rightarrow$ & $\mathrm{NO}_{3}$ & $\begin{array}{l}2.4 \times 10^{-14} \exp (460 / T) \\
6.5 \times 10^{-34} \exp (1335 / T) \\
2.7 \times 10^{-17} \exp (2199 / T)\end{array}$ & 1 \\
\hline K17 & $\mathrm{HO}_{2} \mathrm{NO}_{2}+\mathrm{OH}$ & $\rightarrow$ & $\mathrm{NO}_{2}$ & $1.9 \times 10^{-12} \exp (270 / T)$ & 1 \\
\hline K18 & $\mathrm{HO}_{2} \mathrm{NO}_{2}$ & $\rightarrow$ & $\mathrm{HO}_{2}+\mathrm{NO}_{2}$ & $\begin{array}{l}4.1 \times 10^{-5} \exp (-10650 / T)\left[\mathrm{N}_{2}\right] \\
6.0 \times 10^{15} \exp (-11170 / T) \\
\mathrm{Fc}=0.40\end{array}$ & 1 \\
\hline K19 & $\mathrm{N}_{2} \mathrm{O}_{5}$ & $\rightarrow$ & $\mathrm{NO}_{2}+\mathrm{NO}_{3}$ & $\begin{array}{l}1.3 \times 10^{-3}(T / 300)^{-3.5} \exp (-11000 / T)\left[\mathrm{N}_{2}\right] \\
9.7 \times 10^{14}(T / 300)^{0.1} \exp (-11080 / T) \\
\mathrm{Fc}=0.35\end{array}$ & 1 \\
\hline K20 & $\mathrm{OH}+\mathrm{H}_{2}$ & $\rightarrow$ & $\mathrm{HO}_{2}$ & $7.7 \times 10^{-12} \exp (-2100 / T)$ & 1 \\
\hline K21 & $\mathrm{CH}_{4}+\mathrm{OH}$ & $\rightarrow$ & $\mathrm{CH}_{3} \mathrm{OO}$ & $2.45 \times 10^{-12} \exp (-1775 / T)$ & 2 \\
\hline K22 & $\mathrm{CH}_{3} \mathrm{OO}+\mathrm{HO}_{2}$ & $\rightarrow$ & $\mathrm{CH}_{3} \mathrm{OOH}$ & $\begin{array}{l}3.8 \times 10^{-13} \exp (780 / T) \\
\times(1-1 /(1+498.0 \exp (-1160 / T)))\end{array}$ & 1,3 \\
\hline K23 & $\mathrm{CH}_{3} \mathrm{OO}+\mathrm{HO}_{2}$ & $\rightarrow$ & $\mathrm{HCHO}$ & $\begin{array}{l}3.8 \times 10^{-13} \exp (780 / T) \\
\times(1 /(1+498.0 \exp (-1160 / T)))\end{array}$ & 1,3 \\
\hline K24 & $\mathrm{CH}_{3} \mathrm{OO}+\mathrm{NO}$ & $\rightarrow$ & $\begin{array}{l}0.999\left(\mathrm{HCHO}+\mathrm{HO}_{2}+\mathrm{NO}_{2}\right)+ \\
0.001 \mathrm{CH}_{3} \mathrm{ONO}_{2}\end{array}$ & $2.3 \times 10^{-12} \exp (360 / T)$ & 1,3 \\
\hline K25 & $\mathrm{CH}_{3} \mathrm{OO}+\mathrm{NO}_{2}$ & $\rightarrow$ & $\mathrm{CH}_{3} \mathrm{O}_{2} \mathrm{NO}_{2}$ & $\begin{array}{l}2.5 \times 10^{-30}(T / 300)^{-5.5}\left[\mathrm{~N}_{2}\right] \\
1.8 \times 10^{-11} \\
\mathrm{Fc}=0.36\end{array}$ & 1 \\
\hline K26 & $\mathrm{CH}_{3} \mathrm{OO}+\mathrm{NO}_{3}$ & $\rightarrow$ & $\mathrm{HCHO}+\mathrm{NO}_{2}$ & $1.2 \times 10^{-12}$ & 1 \\
\hline K27 & $\mathrm{CH}_{3} \mathrm{OO}+\mathrm{CH}_{3} \mathrm{OO}$ & $\rightarrow$ & $2 \mathrm{HCHO}+2 \mathrm{HO}_{2}$ & $\begin{array}{l}7.18 \exp (-885 / T) \times \\
1.03 \times 10^{-13} \exp (365 / T)\end{array}$ & 3 \\
\hline
\end{tabular}


Table 2. Continued.

\begin{tabular}{|c|c|c|c|c|c|}
\hline No. & Reactants & & Products* & Rate expression & References \\
\hline K28 & $\mathrm{CH}_{3} \mathrm{OO}+\mathrm{CH}_{3} \mathrm{OO}$ & $\rightarrow$ & $\mathrm{CH}_{3} \mathrm{OH}+\mathrm{HCHO}$ & $\begin{array}{l}(1-7.18 \exp (-885 / T) \times 1.03 \times \\
\left.10^{-13} \exp (365 / T)\right)\end{array}$ & 3 \\
\hline K29 & $\mathrm{CH}_{3} \mathrm{OOH}+\mathrm{OH}$ & $\rightarrow$ & $\mathrm{HCHO}+\mathrm{OH}$ & $0.4 \times 5.3 \times 10^{-12} \exp (190 / T)$ & 1 \\
\hline K30 & $\mathrm{CH}_{3} \mathrm{OOH}+\mathrm{OH}$ & $\rightarrow$ & $\mathrm{CH}_{3} \mathrm{OO}$ & $0.6 \times 5.3 \times 10^{-12} \exp (190 / T)$ & 1 \\
\hline K31 & $\mathrm{CH}_{3} \mathrm{ONO}_{2}+\mathrm{OH}$ & $\rightarrow$ & $\mathrm{HCHO}+\mathrm{NO}_{2}$ & $4.0 \times 10^{-13} \exp (-845 / T)$ & 1 \\
\hline K32 & $\mathrm{CH}_{3} \mathrm{OONO}_{2}$ & $\rightarrow$ & $\mathrm{CH}_{3} \mathrm{O}_{2}+\mathrm{NO}_{2}$ & $\begin{array}{l}9.0 \times 10^{-5} \exp (-9690 / T)\left[\mathrm{N}_{2}\right] \\
1.1 \times 10^{16} \exp (-10560 / T) \\
\mathrm{Fc}=0.36\end{array}$ & 1 \\
\hline K33 & $\mathrm{HCHO}+\mathrm{OH}$ & $\rightarrow$ & $\mathrm{CO}+\mathrm{HO}_{2}$ & $5.4 \times 10^{-12} \exp (135 / T)$ & 1 \\
\hline K34 & $\mathrm{HCHO}+\mathrm{NO}_{3}$ & $\rightarrow$ & $\mathrm{CO}+\mathrm{HO}_{2}+\mathrm{HNO}_{3}$ & $2.0 \times 10^{-12} \exp (-2440 / T)$ & 1 \\
\hline K35 & $\mathrm{CH}_{3} \mathrm{OH}+\mathrm{OH}$ & $\rightarrow$ & $\mathrm{HCHO}+\mathrm{HO}_{2}$ & $2.85 \times 10^{-12} \exp (-345 / T)$ & 1 \\
\hline K36 & $\mathrm{CH}_{3} \mathrm{OH}+\mathrm{NO}_{3}$ & $\rightarrow$ & $\mathrm{HCHO}+\mathrm{HO}_{2}+\mathrm{HNO}_{3}$ & $9.4 \times 10^{-13} \exp (-2650 / T)$ & 1 \\
\hline K37 & $\mathrm{HCOOH}+\mathrm{OH}$ & $\rightarrow$ & $\mathrm{CO}_{2}+\mathrm{HO}_{2}$ & $4.5 \times 10^{-13}$ & 1 \\
\hline K38 & $\mathrm{CO}+\mathrm{OH}$ & $\rightarrow$ & $\mathrm{CO}_{2}+\mathrm{HO}_{2}$ & $\begin{array}{l}5.9 \times 10^{-33}(300 / T)^{1.4} \\
1.1 \times 10^{-12}(300 / T)^{-1.3} \\
1.5 \times 10^{-13}(300 / T)^{-0.6} \\
2.9 \times 10^{9}(300 / T)^{-6.1}\end{array}$ & 2 \\
\hline K39 & $\mathrm{C}_{2} \mathrm{H}_{6}+\mathrm{OH}$ & $\rightarrow$ & $\mathrm{C}_{2} \mathrm{H}_{5} \mathrm{OO}$ & $6.9 \times 10^{-12} \exp (-1000 / T)$ & 1 \\
\hline K40 & $\mathrm{C}_{2} \mathrm{H}_{5} \mathrm{OO}+\mathrm{HO}_{2}$ & $\rightarrow$ & $\mathrm{C}_{2} \mathrm{H}_{5} \mathrm{OOH}$ & $6.4 \times 10^{-13} \exp (710 / T)$ & 1 \\
\hline K41 & $\mathrm{C}_{2} \mathrm{H}_{5} \mathrm{OO}+\mathrm{NO}$ & $\rightarrow$ & $\mathrm{CH}_{3} \mathrm{CHO}+\mathrm{HO}_{2}+\mathrm{NO}_{2}$ & $\begin{array}{l}(1-\mathrm{RTC} 2 \mathrm{P}) \times 2.55 \times \\
10^{-12} \exp (380 / T)\end{array}$ & 1,4 \\
\hline K42 & $\mathrm{C}_{2} \mathrm{H}_{5} \mathrm{OO}+\mathrm{NO}$ & $\rightarrow$ & $\mathrm{C}_{2} \mathrm{H}_{5} \mathrm{ONO}_{2}$ & $\mathrm{RTC} 2 \mathrm{P} \times 2.55 \times 10^{-12} \exp (380 / T)$ & 1,4 \\
\hline K43 & $\mathrm{C}_{2} \mathrm{H}_{5} \mathrm{OO}+\mathrm{CH}_{3} \mathrm{OO}$ & $\rightarrow$ & $\mathrm{CH}_{3} \mathrm{CHO}+\mathrm{HCHO}+2 \mathrm{HO}_{2}$ & $\begin{array}{l}0.8 \times\left(6.4 \times 10^{-14} \times 1.03 \times\right. \\
\left.10^{-13} \exp (365 / T)\right)^{0.5}\end{array}$ & 3 \\
\hline K44 & $\mathrm{C}_{2} \mathrm{H}_{5} \mathrm{OO}+\mathrm{CH}_{3} \mathrm{OO}$ & $\rightarrow$ & $\begin{array}{l}0.5 \mathrm{CH}_{3} \mathrm{CHO} \\
+0.5 \mathrm{CH}_{3} \mathrm{CH}_{2} \mathrm{OH}+\mathrm{CH}_{3} \mathrm{OH}\end{array}$ & $\begin{array}{l}0.2 \times\left(6.4 \times 10^{-14} \times 1.03 \times\right. \\
\left.10^{-13} \exp (365 / T)\right)^{0.5}\end{array}$ & 3 \\
\hline K45 & $\mathrm{C}_{2} \mathrm{H}_{5} \mathrm{OOH}+\mathrm{OH}$ & $\rightarrow$ & $\mathrm{C}_{2} \mathrm{H}_{5} \mathrm{OO}$ & $1.90 \times 10^{-12} \exp (190 / T)$ & 3 \\
\hline K46 & $\mathrm{C}_{2} \mathrm{H}_{5} \mathrm{OOH}+\mathrm{OH}$ & $\rightarrow$ & $\mathrm{CH}_{3} \mathrm{CHO}+\mathrm{OH}$ & $8.01 \times 10^{-12}$ & 3 \\
\hline K47 & $\mathrm{C}_{2} \mathrm{H}_{5} \mathrm{ONO}_{2}+\mathrm{OH}$ & $\rightarrow$ & $\mathrm{CH}_{3} \mathrm{CHO}+\mathrm{NO}_{2}$ & $6.7 \times 10^{-13} \exp (-395 / T)$ & 1 \\
\hline K48 & $\mathrm{CH}_{3} \mathrm{CHO}+\mathrm{OH}$ & $\rightarrow$ & $\mathrm{CH}_{3} \mathrm{C}(\mathrm{O}) \mathrm{OO}$ & $4.7 \times 10^{-12} \exp (345 / T)$ & 1 \\
\hline K49 & $\mathrm{CH}_{3} \mathrm{CHO}+\mathrm{NO}_{3}$ & $\rightarrow$ & $\mathrm{CH}_{3} \mathrm{C}(\mathrm{O}) \mathrm{OO}+\mathrm{HNO}_{3}$ & $1.4 \times 10^{-12} \exp (-1860 / T)$ & 1 \\
\hline K50 & $\mathrm{CH}_{3} \mathrm{C}(\mathrm{O}) \mathrm{OO}+\mathrm{HO}_{2}$ & $\rightarrow$ & $\mathrm{CH}_{3} \mathrm{C}(\mathrm{O}) \mathrm{OOH}$ & $0.41 \times 5.2 \times 10^{-13} \exp (980 / T)$ & 3 \\
\hline K51 & $\mathrm{CH}_{3} \mathrm{C}(\mathrm{O}) \mathrm{OO}+\mathrm{HO}_{2}$ & $\rightarrow$ & $\mathrm{CH}_{3} \mathrm{COOH}+\mathrm{O}_{3}$ & $0.15 \times 5.2 \times 10^{-13} \exp (980 / T)$ & 3 \\
\hline K52 & $\mathrm{CH}_{3} \mathrm{C}(\mathrm{O}) \mathrm{OO}+\mathrm{HO}_{2}$ & $\rightarrow$ & $\mathrm{CH}_{3} \mathrm{OO}+\mathrm{CO}_{2}+\mathrm{OH}$ & $0.44 \times 5.2 \times 10^{-13} \exp (980 / T)$ & 3 \\
\hline K53 & $\mathrm{CH}_{3} \mathrm{C}(\mathrm{O}) \mathrm{OO}+\mathrm{NO}$ & $\rightarrow$ & $\mathrm{CH}_{3} \mathrm{OO}+\mathrm{CO}_{2}+\mathrm{NO}_{2}$ & $7.5 \times 10^{-12} \exp (290 / T)$ & 1 \\
\hline K54 & $\mathrm{CH}_{3} \mathrm{C}(\mathrm{O}) \mathrm{OO}+\mathrm{NO}_{2}$ & $\rightarrow$ & $\mathrm{CH}_{3} \mathrm{C}(\mathrm{O}) \mathrm{OONO}_{2}$ & $\begin{array}{l}3.28 \times 10^{-28}(T / 300)^{-6.87}\left[\mathrm{~N}_{2}\right] \\
1.125 \times 10^{-11}(T / 300)^{-1.105} \\
\mathrm{Fc}=0.3\end{array}$ & 1 \\
\hline K55 & $\mathrm{CH}_{3} \mathrm{C}(\mathrm{O}) \mathrm{OO}+\mathrm{NO}_{3}$ & $\rightarrow$ & $\mathrm{CH}_{3} \mathrm{OO}+\mathrm{NO}_{2}$ & $4.0 \times 10^{-12}$ & 2 \\
\hline K56 & $\mathrm{CH}_{3} \mathrm{C}(\mathrm{O}) \mathrm{OO}+\mathrm{CH}_{3} \mathrm{OO}$ & $\rightarrow$ & $\mathrm{CH}_{3} \mathrm{C}(\mathrm{O}) \mathrm{OOH}+\mathrm{HCHO}$ & $0.9 \times 2.0 \times 10^{-12} \exp (500 / T)$ & 2 \\
\hline K57 & $\mathrm{CH}_{3} \mathrm{C}(\mathrm{O}) \mathrm{OO}+\mathrm{CH}_{3} \mathrm{OO}$ & $\rightarrow$ & $\mathrm{CH}_{3} \mathrm{COOH}+\mathrm{HCHO}$ & $0.1 \times 2.0 \times 10^{-12} \exp (500 / T)$ & 2 \\
\hline K58 & $\mathrm{CH}_{3} \mathrm{C}(\mathrm{O}) \mathrm{OO}+\mathrm{CH}_{3} \mathrm{C}(\mathrm{O}) \mathrm{OO}$ & $\rightarrow$ & $2\left(\mathrm{CH}_{3} \mathrm{OO}+\mathrm{CO}_{2}\right)$ & $2.9 \times 10^{-12} \exp (500 / T)$ & 2 \\
\hline K59 & $\mathrm{CH}_{3} \mathrm{C}(\mathrm{O}) \mathrm{OO}+\mathrm{CH}_{3} \mathrm{COCH}_{2} \mathrm{O}_{2}$ & $\rightarrow$ & $\mathrm{CH}_{3} \mathrm{COOH}+\mathrm{CH}_{3} \mathrm{C}(\mathrm{O}) \mathrm{CHO}$ & $2.5 \times 10^{-12}$ & 2 \\
\hline K60 & $\mathrm{CH}_{3} \mathrm{C}(\mathrm{O}) \mathrm{OO}+\mathrm{CH}_{3} \mathrm{COCH}_{2} \mathrm{O}_{2}$ & $\rightarrow$ & $\begin{array}{l}\mathrm{CH}_{3} \mathrm{OO}+\mathrm{CH}_{3} \mathrm{COCH}_{2} \mathrm{OH} \\
+\mathrm{CO}_{2}\end{array}$ & $2.5 \times 10^{-12}$ & 2 \\
\hline K61 & $\mathrm{CH}_{3} \mathrm{C}(\mathrm{O}) \mathrm{OO}+\mathrm{C}_{2} \mathrm{H}_{5} \mathrm{OO}$ & $\rightarrow$ & $\mathrm{CH}_{3} \mathrm{CHO}+2 \mathrm{CH}_{3} \mathrm{OO}$ & $0.7 \times 4.4 \times 10^{-13} \exp (1070 / T)$ & 1,3 \\
\hline K62 & $\mathrm{CH}_{3} \mathrm{C}(\mathrm{O}) \mathrm{OO}+\mathrm{C}_{2} \mathrm{H}_{5} \mathrm{OO}$ & $\rightarrow$ & $\mathrm{CH}_{3} \mathrm{CHO}+\mathrm{CH}_{3} \mathrm{COOH}$ & $0.3 \times 4.4 \times 10^{-13} \exp (1070 / T)$ & 1,3 \\
\hline K63 & $\mathrm{CH}_{3} \mathrm{C}(\mathrm{O}) \mathrm{OONO}_{2}+\mathrm{OH}$ & $\rightarrow$ & $\mathrm{HCHO}+\mathrm{CO}+\mathrm{NO}_{2}$ & $3.0 \times 10^{-14}$ & 1 \\
\hline K64 & $\mathrm{CH}_{3} \mathrm{C}(\mathrm{O}) \mathrm{OONO}_{2}$ & $\rightarrow$ & $\mathrm{CH}_{3} \mathrm{C}(\mathrm{O}) \mathrm{OO}+\mathrm{NO}_{2}$ & $\begin{array}{l}1.1 \times 10^{-5} \exp (-10100 / T)\left[\mathrm{N}_{2}\right] \\
1.9 \times 10^{17} \exp (-14100 / T) \\
\mathrm{Fc}=0.3\end{array}$ & 1 \\
\hline
\end{tabular}


Table 2. Continued.

\begin{tabular}{|c|c|c|c|c|c|}
\hline No. & Reactants & & Products* & Rate expression & References \\
\hline K65 & $\mathrm{CH}_{3} \mathrm{C}(\mathrm{O}) \mathrm{OONO}_{2}$ & $\rightarrow$ & $\mathrm{CH}_{3} \mathrm{ONO}_{2}+\mathrm{CO}_{2}$ & $2.1 \times 10^{12} \exp (-12525 / T)$ & 5 \\
\hline K66 & $\mathrm{CH}_{3} \mathrm{C}(\mathrm{O}) \mathrm{OOH}+\mathrm{OH}$ & $\rightarrow$ & $\mathrm{CH}_{3} \mathrm{C}(\mathrm{O}) \mathrm{OO}$ & $1.1 \times 10^{-11}$ & 3 \\
\hline K67 & $\mathrm{C}_{2} \mathrm{H}_{4}+\mathrm{OH}$ & $\rightarrow$ & $\mathrm{HOCH}_{2} \mathrm{CH}_{2} \mathrm{OO}$ & $\begin{array}{l}8.6 \times 10^{-29}(T / 300)^{-3.1}\left[\mathrm{~N}_{2}\right] \\
9.0 \times 10^{-12}(T / 300)^{-0.85} \\
\mathrm{Fc}=0.48\end{array}$ & 1 \\
\hline K68 & $\mathrm{C}_{2} \mathrm{H}_{4}+\mathrm{NO}_{3}$ & $\rightarrow$ & $\mathrm{HOCH}_{2} \mathrm{CH}_{2} \mathrm{ONO}_{2}$ & $3.3 \times 10^{-12} \exp (-2880 / T)$ & 1 \\
\hline K69 & $\mathrm{C}_{2} \mathrm{H}_{4}+\mathrm{O}_{3}$ & $\rightarrow$ & $\begin{array}{l}1.37 \mathrm{HCHO}+0.63 \mathrm{CO}+0.13 \\
\mathrm{HO}_{2}+0.13 \mathrm{OH}\end{array}$ & $6.82 \times 10^{-15} \exp (-2500 / T)$ & 1 \\
\hline K70 & $\mathrm{HOCH}_{2} \mathrm{CH}_{2} \mathrm{OO}+\mathrm{HO}_{2}$ & $\rightarrow$ & $\mathrm{HOCH}_{2} \mathrm{CH}_{2} \mathrm{OOH}$ & $1.3 \times 10^{-11}$ & 1 \\
\hline K71 & $\mathrm{HOCH}_{2} \mathrm{CH}_{2} \mathrm{OO}+\mathrm{NO}$ & $\rightarrow$ & $\mathrm{NO}_{2}+2 \mathrm{HCHO}+\mathrm{HO}_{2}$ & $(1-\mathrm{RTC} 2 \mathrm{P}) \times f \times 2.7 \times 10^{-12} \exp (360 / T)$ & 3 \\
\hline K72 & $\mathrm{HOCH}_{2} \mathrm{CH}_{2} \mathrm{OO}+\mathrm{NO}$ & $\rightarrow$ & $\mathrm{NO}_{2}+\mathrm{HOCH}_{2} \mathrm{CHO}+\mathrm{HO}_{2}$ & $\begin{array}{l}(1-\mathrm{RTC} 2 \mathrm{P}) \times(1-f) \times 2.7 \times \\
10^{-12} \exp (360 / T)\end{array}$ & 3 \\
\hline K73 & $\mathrm{HOCH}_{2} \mathrm{CH}_{2} \mathrm{OO}+\mathrm{NO}$ & $\rightarrow$ & $\mathrm{HOCH}_{2} \mathrm{CH}_{2} \mathrm{ONO}_{2}$ & $\mathrm{RTC} 2 \mathrm{P} \times 2.7 \times 10^{-12} \exp (360 / T)$ & 1 \\
\hline K74 & $\mathrm{HOCH}_{2} \mathrm{CH}_{2} \mathrm{OO}+\mathrm{CH}_{3} \mathrm{OO}$ & $\rightarrow$ & $\mathrm{HOCH}_{2} \mathrm{CHO}+\mathrm{HCHO}+2 \mathrm{HO}_{2}$ & $\begin{array}{l}0.8 \times\left(7.8 \times 10^{14} \exp (1000 / T) \times 1.03 \times\right. \\
\left.10^{-13} \exp (365 / T)\right)^{0.5}\end{array}$ & 3 \\
\hline K75 & $\mathrm{HOCH}_{2} \mathrm{CH}_{2} \mathrm{OO}+\mathrm{CH}_{3} \mathrm{OO}$ & $\rightarrow$ & $\mathrm{HOCH}_{2} \mathrm{CHO}+\mathrm{CH}_{3} \mathrm{OH}$ & $\begin{array}{l}0.2 \times\left(7.8 \times 10^{14} \exp (1000 / T)\right. \\
\left.\times 1.03 \times 10^{-13} \exp (365 / T)\right)^{0.5}\end{array}$ & 3 \\
\hline K76 & $\mathrm{HOCH}_{2} \mathrm{CH}_{2} \mathrm{OOH}+\mathrm{OH}$ & $\rightarrow$ & $\mathrm{HOCH}_{2} \mathrm{CH}_{2} \mathrm{OO}$ & K45 & \\
\hline K77 & $\mathrm{HOCH}_{2} \mathrm{CH}_{2} \mathrm{OOH}+\mathrm{OH}$ & $\rightarrow$ & $\mathrm{HOCH}_{2} \mathrm{CHO}+\mathrm{OH}$ & $1.38 \times 10^{-11}$ & 3 \\
\hline K78 & $\mathrm{HOCH}_{2} \mathrm{CH}_{2} \mathrm{ONO}_{2}+\mathrm{OH}$ & $\rightarrow$ & $\mathrm{HOCH}_{2} \mathrm{CHO}+\mathrm{NO}_{2}$ & $8.4 \times 10^{-13}$ & 3 \\
\hline K79 & $\mathrm{C}_{2} \mathrm{H}_{2}+\mathrm{OH}$ & $\rightarrow$ & $\begin{array}{l}0.636(\mathrm{CHOCHO}+\mathrm{OH}) \\
+0.364\left(\mathrm{HCOOH}+\mathrm{CO}+\mathrm{HO}_{2}\right)\end{array}$ & $\begin{array}{l}5.0 \times 10^{-30}(T / 300)^{-1.5}\left[\mathrm{~N}_{2}\right] \\
1.0 \times 10^{-12} \\
\mathrm{Fc}=0.37\end{array}$ & 1 \\
\hline K80 & $\mathrm{C}_{2} \mathrm{H}_{2}+\mathrm{NO}_{3}$ & $\rightarrow$ & $\begin{array}{l}0.635 \mathrm{CHOCHO} \\
+0.365(\mathrm{HCOOH}+\mathrm{CO}) \\
+\mathrm{HNO}_{3}\end{array}$ & $1.0 \times 10^{-16}$ & 1 \\
\hline K81 & $\mathrm{C}_{2} \mathrm{H}_{2}+\mathrm{O}_{3}$ & $\rightarrow$ & $\begin{array}{l}0.635 \mathrm{CHOCHO} \\
+0.365(\mathrm{HCOOH}+\mathrm{CO})\end{array}$ & $1.0 \times 10^{-20}$ & 1 \\
\hline K82 & $\mathrm{HOCH}_{2} \mathrm{CHO}+\mathrm{OH}$ & $\rightarrow$ & $\mathrm{HCHO}+\mathrm{CO}_{2}$ & $6.4 \times 10^{-12}$ & 1 \\
\hline K83 & $\mathrm{HOCH}_{2} \mathrm{CHO}+\mathrm{OH}$ & $\rightarrow$ & $\mathrm{CHOCHO}+\mathrm{HO}_{2}$ & $1.6 \times 10^{-12}$ & 1 \\
\hline K84 & $\mathrm{CHOCHO}+\mathrm{OH}$ & $\rightarrow$ & $2 \mathrm{CO}+\mathrm{HO}_{2}$ & $3.1 \times 10^{-12} \exp (340 / T)$ & 1 \\
\hline K85 & $\mathrm{CHOCHO}+\mathrm{NO}_{3}$ & $\rightarrow$ & $2 \mathrm{CO}+\mathrm{HO}_{2}+\mathrm{HNO}_{3}$ & $4.0 \times 10^{-16}$ & 1 \\
\hline K86 & $\mathrm{CH}_{3} \mathrm{CH}_{2} \mathrm{OH}+\mathrm{OH}$ & $\rightarrow$ & $\mathrm{CH}_{3} \mathrm{OO}+\mathrm{CO}_{2}$ & $4.0 \times 10^{-14} \exp (850 / T)$ & 1 \\
\hline K87 & $\mathrm{CH}_{3} \mathrm{COOH}+\mathrm{OH}$ & $\rightarrow$ & $\begin{array}{l}0.95\left(\mathrm{CH}_{3} \mathrm{CHO}+\mathrm{HO}_{2}\right) \\
+0.05 \mathrm{HOCH}_{2} \mathrm{CH}_{2} \mathrm{OO}\end{array}$ & $3.0 \times 10^{-12} \exp (20 / T)$ & 1 \\
\hline K88 & $\mathrm{C}_{3} \mathrm{H}_{8}+\mathrm{OH}$ & $\rightarrow$ & $\begin{array}{l}0.264 n-\mathrm{C}_{3} \mathrm{H}_{7} \mathrm{O}_{2} \\
+0.736 i-\mathrm{C}_{3} \mathrm{H}_{7} \mathrm{O}_{2}\end{array}$ & $7.6 \times 10^{-12} \exp (-585 / T)$ & 1,3 \\
\hline K89 & $n-\mathrm{C}_{3} \mathrm{H}_{7} \mathrm{O}_{2}+\mathrm{HO}_{2}$ & $\rightarrow$ & $n-\mathrm{C}_{3} \mathrm{H}_{7} \mathrm{OOH}$ & $0.52 \times 2.91 \times 10^{-13} \exp (1300 / T)$ & 3 \\
\hline K90 & $n-\mathrm{C}_{3} \mathrm{H}_{7} \mathrm{O}_{2}+\mathrm{NO}$ & $\rightarrow$ & $\mathrm{C}_{2} \mathrm{H}_{5} \mathrm{CHO}+\mathrm{HO}_{2}+\mathrm{NO}_{2}$ & $(1-\mathrm{RTC} 3 \mathrm{P}) \times 2.9 \times 10^{-12} \exp (350 / T)$ & 1,4 \\
\hline K91 & $n-\mathrm{C}_{3} \mathrm{H}_{7} \mathrm{O}_{2}+\mathrm{NO}$ & $\rightarrow$ & $n-\mathrm{C}_{3} \mathrm{H}_{7} \mathrm{ONO}_{2}$ & RTC3P $\times 2.9 \times 10^{-12} \exp (350 / T)$ & 1,4 \\
\hline K92 & $n-\mathrm{C}_{3} \mathrm{H}_{7} \mathrm{O}_{2}+\mathrm{CH}_{3} \mathrm{OO}$ & $\rightarrow$ & $\mathrm{C}_{2} \mathrm{H}_{5} \mathrm{CHO}+\mathrm{CH}_{3} \mathrm{OH}$ & $0.8 \times\left(3.5 \times 10^{-13} \times 3.0 \times 10^{13}\right)^{0.5}$ & 3 \\
\hline K93 & $n-\mathrm{C}_{3} \mathrm{H}_{7} \mathrm{O}_{2}+\mathrm{CH}_{3} \mathrm{OO}$ & $\rightarrow$ & $\mathrm{C}_{2} \mathrm{H}_{5} \mathrm{CHO}+\mathrm{HCHO}+2 \mathrm{HO}_{2}$ & $0.2 \times\left(3.5 \times 10^{-13} \times 3.0 \times 10^{13}\right)^{0.5}$ & 3 \\
\hline K94 & $n-\mathrm{C}_{3} \mathrm{H}_{7} \mathrm{OOH}+\mathrm{OH}$ & $\rightarrow$ & $n-\mathrm{C}_{3} \mathrm{H}_{7} \mathrm{O}_{2}$ & K76 & \\
\hline K95 & $n-\mathrm{C}_{3} \mathrm{H}_{7} \mathrm{OOH}+\mathrm{OH}$ & $\rightarrow$ & $\mathrm{C}_{2} \mathrm{H}_{5} \mathrm{CHO}+\mathrm{OH}$ & $1.66 \times 10^{-11}$ & 3 \\
\hline K96 & $n-\mathrm{C}_{3} \mathrm{H}_{7} \mathrm{ONO}_{2}+\mathrm{OH}$ & $\rightarrow$ & $\mathrm{C}_{2} \mathrm{H}_{5} \mathrm{CHO}+\mathrm{NO}_{2}$ & $5.8 \times 10^{-13}$ & 1 \\
\hline K97 & $i-\mathrm{C}_{3} \mathrm{H}_{7} \mathrm{O}_{2}+\mathrm{HO}_{2}$ & $\rightarrow$ & $i-\mathrm{C}_{3} \mathrm{H}_{7} \mathrm{OOH}$ & K89 & \\
\hline K98 & $i-\mathrm{C}_{3} \mathrm{H}_{7} \mathrm{O}_{2}+\mathrm{NO}$ & $\rightarrow$ & $\mathrm{CH}_{3} \mathrm{COCH}_{3}+\mathrm{HO}_{2}+\mathrm{NO}_{2}$ & $(1-\mathrm{RTC} 3 \mathrm{~S}) \times 2.7 \times 10^{-12} \exp (360 / T)$ & 1,4 \\
\hline K99 & $i-\mathrm{C}_{3} \mathrm{H}_{7} \mathrm{O}_{2}+\mathrm{NO}$ & $\rightarrow$ & $i-\mathrm{C}_{3} \mathrm{H}_{7} \mathrm{ONO}_{2}$ & $\mathrm{RTC} 3 \mathrm{~S} \times 2.7 \times 10^{-12} \exp (360 / T)$ & 1,4 \\
\hline K100 & $i-\mathrm{C}_{3} \mathrm{H}_{7} \mathrm{O}_{2}+\mathrm{CH}_{3} \mathrm{OO}$ & $\rightarrow$ & $\mathrm{CH}_{3} \mathrm{COCH}_{3}+\mathrm{HCHO}+2 \mathrm{HO}_{2}$ & $\begin{array}{l}0.8 \times\left(1.03 \times 10^{-13} \exp (365 / T) \times\right. \\
\left.1.6 \times 10^{-12} \exp (-2200 / T)\right)^{0.5}\end{array}$ & 3 \\
\hline K101 & $i-\mathrm{C}_{3} \mathrm{H}_{7} \mathrm{O}_{2}+\mathrm{CH}_{3} \mathrm{OO}$ & $\rightarrow$ & $\mathrm{CH}_{3} \mathrm{COCH}_{3}+\mathrm{CH}_{3} \mathrm{OH}$ & $\begin{array}{l}0.2 \times\left(1.03 \times 10^{-13} \exp (365 / T) \times\right. \\
\left.1.6 \times 10^{-12} \exp (-2200 / T)\right)^{0.5}\end{array}$ & 3 \\
\hline
\end{tabular}


Table 2. Continued.

\begin{tabular}{|c|c|c|c|c|c|}
\hline No. & Reactants & & Products* & Rate expression & References \\
\hline K102 & $i-\mathrm{C}_{3} \mathrm{H}_{7} \mathrm{OOH}+\mathrm{OH}$ & $\rightarrow$ & $i-\mathrm{C}_{3} \mathrm{H}_{7} \mathrm{O}_{2}$ & $1.9 \times 10^{-12} \exp (190 / T)$ & 3 \\
\hline K103 & $i-\mathrm{C}_{3} \mathrm{H}_{7} \mathrm{OOH}+\mathrm{OH}$ & $\rightarrow$ & $\mathrm{CH}_{3} \mathrm{COCH}_{3}+\mathrm{OH}$ & $1.66 \times 10^{-11}$ & 3 \\
\hline K104 & $i-\mathrm{C}_{3} \mathrm{H}_{7} \mathrm{ONO}_{2}+\mathrm{OH}$ & $\rightarrow$ & $\mathrm{CH}_{3} \mathrm{COCH}_{3}+\mathrm{NO}_{2}$ & $6.2 \times 10^{-13} \exp (-230 / T)$ & 1 \\
\hline K105 & $\mathrm{C}_{2} \mathrm{H}_{5} \mathrm{CHO}+\mathrm{OH}$ & $\rightarrow$ & $\mathrm{CH}_{3} \mathrm{C}(\mathrm{O}) \mathrm{OO}+\mathrm{CO}$ & $4.9 \times 10^{-12} \exp (405 / T)$ & 1 \\
\hline K106 & $\mathrm{C}_{2} \mathrm{H}_{5} \mathrm{CHO}+\mathrm{NO}_{3}$ & $\rightarrow$ & $\mathrm{CH}_{3} \mathrm{C}(\mathrm{O}) \mathrm{OO}+\mathrm{CO}+\mathrm{HNO}_{3}$ & $6.3 \times 10^{-15}$ & 1 \\
\hline K107 & $\mathrm{CH}_{3} \mathrm{COCH}_{3}+\mathrm{OH}$ & $\rightarrow$ & $\mathrm{CH}_{3} \mathrm{COCH}_{2} \mathrm{OO}$ & $\begin{array}{l}8.8 \times 10^{-12} \exp (-1320 / T)+ \\
1.7 \times 10^{-14} \exp (423 / T)\end{array}$ & 1 \\
\hline K108 & $\mathrm{CH}_{3} \mathrm{COCH}_{2} \mathrm{OO}+\mathrm{NO}$ & $\rightarrow$ & $\mathrm{CH}_{3} \mathrm{C}(\mathrm{O}) \mathrm{CHO}+\mathrm{NO}_{2}+\mathrm{HO}_{2}$ & $2.7 \times 10^{-13} \exp (360 / T)$ & 3 \\
\hline K109 & $\mathrm{CH}_{3} \mathrm{COCH}_{2} \mathrm{OO}+\mathrm{HO}_{2}$ & $\rightarrow$ & $\mathrm{CH}_{3} \mathrm{COCH}_{2} \mathrm{OOH}$ & $1.36 \times 10^{-13} \exp (1250 / T)$ & 3 \\
\hline K110 & $\mathrm{CH}_{3} \mathrm{COCH}_{2} \mathrm{OOH}+\mathrm{OH}$ & $\rightarrow$ & $\begin{array}{l}0.7 \mathrm{CH}_{3} \mathrm{C}(\mathrm{O}) \mathrm{CHO} \\
+0.3 \mathrm{CH}_{3} \mathrm{COCH}_{2} \mathrm{OO}+\mathrm{OH}\end{array}$ & $1.90 \times 10^{-12} \exp (190 / T)$ & 3 \\
\hline K111 & $\mathrm{C}_{3} \mathrm{H}_{6}+\mathrm{OH}$ & $\rightarrow$ & $\mathrm{HOC}_{3} \mathrm{H}_{6} \mathrm{OO}$ & $\begin{array}{l}8 \times 10^{-27}(T / 300)^{-3.5}\left[\mathrm{~N}_{2}\right] \\
3.0 \times 10^{-11}(T / 300)^{-1.0} \\
\mathrm{Fc}=0.5\end{array}$ & 1 \\
\hline K112 & $\mathrm{C}_{3} \mathrm{H}_{6}+\mathrm{NO}_{3}$ & $\rightarrow$ & $\begin{array}{l}0.35 n-\mathrm{C}_{3} \mathrm{H}_{7} \mathrm{ONO}_{2} \\
+0.65 i-\mathrm{C}_{3} \mathrm{H}_{7} \mathrm{ONO}_{2}\end{array}$ & $4.6 \times 10^{-13} \exp (-1155 / T)$ & 1,3 \\
\hline K113 & $\mathrm{C}_{3} \mathrm{H}_{6}+\mathrm{O}_{3}$ & $\rightarrow$ & $\begin{array}{l}0.62 \mathrm{HCHO}+0.62 \mathrm{CH}_{3} \mathrm{CHO} \\
+0.38 \mathrm{CH}_{3} \mathrm{OO}+0.56 \mathrm{CO} \\
+0.36 \mathrm{HO}_{2}+0.36 \quad \mathrm{OH}+0.2 \\
\mathrm{CO}_{2}\end{array}$ & $5.77 \times 10^{-15} \exp (-1880 / T)$ & 1,3 \\
\hline K114 & $\mathrm{HOC}_{3} \mathrm{H}_{6} \mathrm{OOH}+\mathrm{OH}$ & $\rightarrow$ & $\begin{array}{l}0.928 \mathrm{CH}_{3} \mathrm{COCH} \mathrm{CH}_{2} \mathrm{OH}+0.072 \\
\mathrm{HOC}_{3} \mathrm{H}_{6} \mathrm{OO}+0.928 \mathrm{OH}\end{array}$ & $2.44 \times 10^{-11}+1.9 \times 10^{-12} \exp (190 / T)$ & 3 \\
\hline K115 & $\mathrm{HOC}_{3} \mathrm{H}_{6} \mathrm{OO}+\mathrm{HO}_{2}$ & $\rightarrow$ & $\mathrm{HOC}_{3} \mathrm{H}_{6} \mathrm{OOH}$ & K89 & 3 \\
\hline K116 & $\mathrm{HOC}_{3} \mathrm{H}_{6} \mathrm{OO}+\mathrm{NO}$ & $\rightarrow$ & $\begin{array}{l}\mathrm{CH}_{3} \mathrm{CHO}+\mathrm{HCHO}+\mathrm{HO}_{2} \\
+\mathrm{NO}_{2}\end{array}$ & $\begin{array}{l}(1-0.35 \mathrm{RTC} 3 \mathrm{P}-0.65 \mathrm{RTC} 3 \mathrm{~S}) \times \\
2.55 \times 10^{-12} \exp (380 / T)\end{array}$ & 1,3 \\
\hline K117 & $\mathrm{HOC}_{3} \mathrm{H}_{6} \mathrm{OO}+\mathrm{NO}$ & $\rightarrow$ & $\begin{array}{l}0.35 n-\mathrm{C}_{3} \mathrm{H}_{7} \mathrm{ONO}_{2} \\
+0.65 i-\mathrm{C}_{3} \mathrm{H}_{7} \mathrm{ONO}_{2}\end{array}$ & $\begin{array}{l}(0.35 \mathrm{RTC} 3 \mathrm{P}+0.65 \mathrm{RTC} 3 \mathrm{~S}) \times \\
2.55 \times 10^{-12} \exp (380 / T)\end{array}$ & 1,3 \\
\hline K118 & $\mathrm{HOC}_{3} \mathrm{H}_{6} \mathrm{OO}+\mathrm{CH}_{3} \mathrm{OO}$ & $\rightarrow$ & $\mathrm{CH}_{3} \mathrm{CHO}+2 \mathrm{HCHO}+2 \mathrm{HO}_{2}$ & $0.8 \times 6.0 \times 10^{-13}$ & 3 \\
\hline K119 & $\mathrm{HOC}_{3} \mathrm{H}_{6} \mathrm{OO}+\mathrm{CH}_{3} \mathrm{OO}$ & $\rightarrow$ & $\mathrm{CH}_{3} \mathrm{COCH}_{2} \mathrm{OH}+\mathrm{CH}_{3} \mathrm{OH}$ & $0.2 \times 6.0 \times 10^{-13}$ & 3 \\
\hline K120 & $\mathrm{CH}_{3} \mathrm{COCH}_{2} \mathrm{OH}+\mathrm{OH}$ & $\rightarrow$ & $\mathrm{CH}_{3} \mathrm{C}(\mathrm{O}) \mathrm{CHO}+\mathrm{HO}_{2}$ & $1.6 \times 10^{-12} \exp (305 / T)$ & 1 \\
\hline K121 & $\mathrm{CH}_{3} \mathrm{C}(\mathrm{O}) \mathrm{CHO}+\mathrm{OH}$ & $\rightarrow$ & $\mathrm{CH}_{3} \mathrm{C}(\mathrm{O}) \mathrm{OO}+\mathrm{CO}$ & $1.9 \times 10^{-12} \exp (575 / T)$ & 1 \\
\hline K122 & $\mathrm{CH}_{3} \mathrm{C}(\mathrm{O}) \mathrm{CHO}+\mathrm{NO}_{3}$ & $\rightarrow$ & $\mathrm{CH}_{3} \mathrm{C}(\mathrm{O}) \mathrm{OO}+\mathrm{CO}+\mathrm{HNO}_{3}$ & $5.0 \times 10^{-16}$ & 1 \\
\hline K123 & $\mathrm{CH}_{3} \mathrm{C}(\mathrm{O}) \mathrm{COOH}+\mathrm{OH}$ & $\rightarrow$ & $\mathrm{CH}_{3} \mathrm{C}(\mathrm{O}) \mathrm{OO}+\mathrm{CO}_{2}$ & $8.0 \times 10^{-13}$ & 3 \\
\hline K124 & $\mathrm{C}_{4} \mathrm{H}_{10}+\mathrm{OH}$ & $\rightarrow$ & $\mathrm{C}_{4} \mathrm{H}_{9} \mathrm{OO}$ & $9.8 \times 10^{-12} \exp (-425 / T)$ & 3 \\
\hline K125 & $\mathrm{C}_{4} \mathrm{H}_{10}+\mathrm{NO}_{3}$ & $\rightarrow$ & $\mathrm{C}_{4} \mathrm{H}_{9} \mathrm{OO}+\mathrm{HNO}_{3}$ & $2.8 \times 10^{-12} \exp (-3280 / T)$ & 1 \\
\hline K126 & $\mathrm{C}_{4} \mathrm{H}_{9} \mathrm{OO}+\mathrm{HO}_{2}$ & $\rightarrow$ & $\mathrm{C}_{4} \mathrm{H}_{9} \mathrm{OOH}$ & $0.625 \times 2.91 \times 10^{-13} \exp (1300 / T)$ & 3 \\
\hline K127 & $\mathrm{C}_{4} \mathrm{H}_{9} \mathrm{OO}+\mathrm{NO}$ & $\rightarrow$ & $\begin{array}{l}\mathrm{NO}_{2}+0.67\left(\mathrm{CH}_{3} \mathrm{CH}_{2} \mathrm{COCH}_{3}\right. \\
\left.+\mathrm{HO}_{2}\right)+0.33\left(\mathrm{C}_{2} \mathrm{H}_{5} \mathrm{OO}\right. \\
\left.+\mathrm{CH}_{3} \mathrm{CHO}\right)\end{array}$ & $(1-\mathrm{RTC} 4 \mathrm{P}) \times 8.3 \times 10^{-12}$ & 1,4 \\
\hline K128 & $\mathrm{C}_{4} \mathrm{H}_{9} \mathrm{OO}+\mathrm{NO}$ & $\rightarrow$ & $\mathrm{C}_{4} \mathrm{H}_{9} \mathrm{ONO}_{2}$ & $\mathrm{RTC} 4 \mathrm{P} \times 8.3 \times 10^{-12}$ & 1,4 \\
\hline K129 & $\mathrm{C}_{4} \mathrm{H}_{9} \mathrm{OO}+\mathrm{CH}_{3} \mathrm{OO}$ & $\rightarrow$ & $\begin{array}{l}\mathrm{HCHO}+\mathrm{HO}_{2} \\
+0.67\left(\mathrm{CH}_{3} \mathrm{CH}_{2} \mathrm{C}(\mathrm{O}) \mathrm{CH}_{3}\right. \\
\left.+\mathrm{HO}_{2}\right)+0.33\left(\mathrm{CH}_{3} \mathrm{CHO}\right. \\
\left.+\mathrm{CH}_{3} \mathrm{CH}_{2} \mathrm{OO}\right)\end{array}$ & $0.8 \times 1.3 \times 10^{-12}$ & 3 \\
\hline K130 & $\mathrm{C}_{4} \mathrm{H}_{9} \mathrm{OO}+\mathrm{CH}_{3} \mathrm{OO}$ & $\rightarrow$ & $\mathrm{CH}_{3} \mathrm{CH}_{2} \mathrm{COCH}_{3}+\mathrm{CH}_{3} \mathrm{OH}$ & $0.2 \times 1.3 \times 10^{-12}$ & 3 \\
\hline K131 & $\mathrm{C}_{4} \mathrm{H}_{9} \mathrm{OOH}+\mathrm{OH}$ & $\rightarrow$ & $\mathrm{C}_{4} \mathrm{H}_{9} \mathrm{OO}$ & $1.90 \times 10^{-12} \exp (190 / T)$ & 3 \\
\hline K132 & $\mathrm{C}_{4} \mathrm{H}_{9} \mathrm{OOH}+\mathrm{OH}$ & $\rightarrow$ & $\mathrm{CH}_{3} \mathrm{CH}_{2} \mathrm{COCH}_{3}+\mathrm{OH}$ & $2.15 \times 10^{-11}$ & 3 \\
\hline K133 & $\mathrm{C}_{4} \mathrm{H}_{9} \mathrm{ONO}_{2}+\mathrm{OH}$ & $\rightarrow$ & $\mathrm{CH}_{3} \mathrm{CH}_{2} \mathrm{COCH}_{3}+\mathrm{NO}_{2}$ & $8.6 \times 10^{-13}$ & 1 \\
\hline K134 & $\mathrm{CH}_{3} \mathrm{CH}_{2} \mathrm{COCH}_{3}+\mathrm{OH}$ & $\rightarrow$ & $\mathrm{CH}_{3} \mathrm{CH}(\mathrm{OO}) \mathrm{COCH}_{3}$ & $1.5 \times 10^{-12} \exp (-90 / T)$ & 1 \\
\hline K135 & $\mathrm{CH}_{3} \mathrm{CH}(\mathrm{OO}) \mathrm{COCH}_{3}+\mathrm{HO}_{2}$ & $\rightarrow$ & $\mathrm{CH}_{3} \mathrm{CH}(\mathrm{OOH}) \mathrm{COCH}_{3}$ & K126 & \\
\hline K136 & $\mathrm{CH}_{3} \mathrm{CH}(\mathrm{OO}) \mathrm{COCH}_{3}+\mathrm{NO}$ & $\rightarrow$ & $\begin{array}{l}\mathrm{CH}_{3} \mathrm{CHO}+\mathrm{CH}_{3} \mathrm{C}(\mathrm{O}) \mathrm{OO} \\
+\mathrm{NO}_{2}\end{array}$ & $(1-\mathrm{RTC} 4 \mathrm{~S}) \times 2.55 \times 10^{-12} \exp (380 / T)$ & 1,4 \\
\hline K137 & $\mathrm{CH}_{3} \mathrm{CH}(\mathrm{OO}) \mathrm{COCH}_{3}+\mathrm{NO}$ & $\rightarrow$ & $\mathrm{CH}_{3} \mathrm{CH}\left(\mathrm{ONO}_{2}\right) \mathrm{COCH}_{3}$ & $\mathrm{RTC} 4 \mathrm{~S} \times 2.55 \times 10^{-12} \exp (380 / T)$ & 1,4 \\
\hline
\end{tabular}


Table 2. Continued.

\begin{tabular}{|c|c|c|c|c|c|}
\hline No. & Reactants & & Products* & Rate expression & References \\
\hline K138 & $\mathrm{CH}_{3} \mathrm{CH}(\mathrm{OOH}) \mathrm{COCH}_{3}+\mathrm{OH}$ & $\rightarrow$ & $\mathrm{CH}_{3} \mathrm{CH}(\mathrm{OO}) \mathrm{COCH}_{3}$ & K131 & \\
\hline K139 & $\mathrm{CH}_{3} \mathrm{CH}(\mathrm{OOH}) \mathrm{COCH}_{3}+\mathrm{OH}$ & $\rightarrow$ & $\mathrm{CH}_{3} \mathrm{C}(\mathrm{O}) \mathrm{C}(\mathrm{O}) \mathrm{CH}_{3}+\mathrm{OH}$ & $1.88 \times 10^{-11}$ & 3 \\
\hline K140 & $\mathrm{CH}_{3} \mathrm{CH}\left(\mathrm{ONO}_{2}\right) \mathrm{COCH}_{3}+\mathrm{OH}$ & $\rightarrow$ & $\mathrm{CH}_{3} \mathrm{C}(\mathrm{O}) \mathrm{C}(\mathrm{O}) \mathrm{CH}_{3}+\mathrm{NO}_{2}$ & $1.2 \times 10^{-12}$ & 1 \\
\hline K141 & $\mathrm{ISOP}+\mathrm{OH}$ & $\rightarrow$ & $\begin{array}{l}0.98 \text { ISOPOO }+0.0003 \text { ELVOC } \\
+0.007 \text { SVOC }\end{array}$ & $2.7 \times 10^{-11} \exp (390 / T)$ & 1,3 \\
\hline K142 & $\mathrm{ISOP}+\mathrm{NO}_{3}$ & $\rightarrow$ & ISOPONO$_{2}$ & $2.95 \times 10^{-12} \exp (-450 / T)$ & 1,3 \\
\hline K143 & $\mathrm{ISOP}+\mathrm{O}_{3}$ & $\rightarrow$ & $\begin{array}{l}0.98 \times(0.3 \mathrm{MACR} \\
+0.3 \mathrm{MACROO}+0.2 \mathrm{MVK}+0.2 \\
\mathrm{MVKOO}+0.78 \mathrm{HCHO}+0.22 \mathrm{CO} \\
\left.+0.125 \mathrm{HO}_{2}+0.125 \mathrm{OH}\right) \\
+0.0001 \mathrm{ELVOC}+0.009 \mathrm{SVOC}\end{array}$ & $1.05 \times 10^{-14} \exp (-2000 / T)$ & 1,3 \\
\hline K144 & $\mathrm{ISOPOO}+\mathrm{HO}_{2}$ & $\rightarrow$ & ISOPOOH & $2.06 \times 10^{-13} \exp (1300 / T)$ & 3,7 \\
\hline K145 & $\mathrm{ISOPOO}+\mathrm{NO}$ & $\rightarrow$ & $\begin{array}{l}\mathrm{HCHO}+0.64 \mathrm{MVK}+0.36 \mathrm{MACR} \\
+\mathrm{HO}_{2}+\mathrm{NO}_{2}\end{array}$ & $\begin{array}{l}(1-\mathrm{RTC} 5 \mathrm{~S}) \times 2.7 \times 10^{-12} \\
\exp (360 / T)\end{array}$ & 3 \\
\hline K146 & $\mathrm{ISOPOO}+\mathrm{NO}$ & $\rightarrow$ & $\mathrm{ISOPONO}_{2}$ & RTC5S $\times 2.7 \times 10^{-12} \exp (360 / T)$ & 3 \\
\hline K147 & $\mathrm{ISOPOO}+\mathrm{NO}_{3}$ & $\rightarrow$ & $\begin{array}{l}\mathrm{HCHO}+0.64 \mathrm{MVK}+0.36 \mathrm{MACR} \\
+\mathrm{HO}_{2}+\mathrm{NO}_{2}\end{array}$ & $2.3 \times 10^{-12}$ & 3 \\
\hline K148 & $\mathrm{ISOPOO}+\mathrm{CH}_{3} \mathrm{OO}$ & $\rightarrow$ & $\begin{array}{l}0.64 \mathrm{MVK}+0.36 \mathrm{MACR} \\
+2 \mathrm{HCHO}+2 \mathrm{HO}_{2}\end{array}$ & $0.8 \times 2.65 \times 10^{-12}$ & 3 \\
\hline K149 & $\mathrm{ISOPOO}+\mathrm{CH}_{3} \mathrm{OO}$ & $\rightarrow$ & $\begin{array}{l}0.64 \mathrm{MVK}+0.36 \mathrm{MACR} \\
+\mathrm{HCHO}+\mathrm{CH}_{3} \mathrm{OH}\end{array}$ & $0.2 \times 2.65 \times 10^{-12}$ & 3 \\
\hline K150 & ISOPOO & $\rightarrow$ & $\mathrm{HPALD}+\mathrm{HO}_{2}$ & $4.12 \times 10^{8} \exp (-7700 / T)$ & 6,7 \\
\hline K151 & $\mathrm{ISOPOOH}+\mathrm{OH}$ & $\rightarrow$ & $\mathrm{IEPOX}+\mathrm{OH}$ & $1.9 \times 10^{-11} \exp (-390 / T)$ & 8 \\
\hline K152 & $\mathrm{ISOPOOH}+\mathrm{OH}$ & $\rightarrow$ & ISOPOO & $0.7 \times 3.8 \times 10^{-12} \exp (-200 / T)$ & 8 \\
\hline K153 & $\mathrm{ISOPOOH}+\mathrm{OH}$ & $\rightarrow$ & $\begin{array}{l}0.64 \mathrm{CH}_{3} \mathrm{C}(\mathrm{O}) \mathrm{CHO} \\
+0.64 \mathrm{HOCH}_{2} \mathrm{CHO} \\
+0.36 \mathrm{HOCH}_{2} \mathrm{C}(\mathrm{O}) \mathrm{CH}_{3} \\
+0.36 \mathrm{CHOCHO}+\mathrm{OH}\end{array}$ & $0.3 \times 3.8 \times 10^{-12} \exp (-200 / T)$ & 8,9 \\
\hline K154 & $\mathrm{ISOPONO}_{2}+\mathrm{OH}$ & $\rightarrow$ & $\begin{array}{l}0.64 \mathrm{CH}_{3} \mathrm{C}(\mathrm{O}) \mathrm{CHO} \\
+0.64 \mathrm{HOCH}_{2} \mathrm{CHO} \\
+0.36 \mathrm{HOCH}_{2} \mathrm{C}(\mathrm{O}) \mathrm{CH}_{3} \\
+0.36 \mathrm{CHOCHO}+\mathrm{NO}_{2}\end{array}$ & $1.77 \times 10^{-11} \exp (-500 / T)$ & 8 \\
\hline K155 & $\mathrm{HPALD}+\mathrm{OH}$ & $\rightarrow$ & $\begin{array}{l}0.5 \mathrm{HOCH}_{2} \mathrm{C}(\mathrm{O}) \mathrm{CH}_{3} \\
+0.5 \mathrm{CH}_{3} \mathrm{C}(\mathrm{O}) \mathrm{CHO} \\
+0.25 \mathrm{HOCH}_{2} \mathrm{CHO}+0.25 \mathrm{CHO}- \\
\mathrm{CHO}+\mathrm{HCHO}+\mathrm{HO}_{2}+\mathrm{OH}\end{array}$ & $4.6 \times 10^{-11}$ & 6 \\
\hline K156 & IEPOX + OH & $\rightarrow$ & IEPOXOO & $5.78 \times 10^{-11} \exp (-400 / T)$ & 8 \\
\hline K157 & $\mathrm{IEPOXOO}+\mathrm{HO}_{2}$ & $\rightarrow$ & $\begin{array}{l}0.725 \mathrm{HOCH}_{2} \mathrm{C}(\mathrm{O}) \mathrm{CH}_{3} \\
+0.275 \mathrm{HOCH}_{2} \mathrm{CHO} \\
+0.275 \mathrm{CHOCHO}^{-} \\
+0.275 \mathrm{CH}_{3} \mathrm{C}(\mathrm{O}) \mathrm{CHO}+1.125 \\
\mathrm{OH}+0.825 \mathrm{HO}_{2} \\
+0.2 \mathrm{CO}_{2}+0.375 \mathrm{HCHO}+0.074 \\
\mathrm{HCOOH}+0.251 \mathrm{CO}\end{array}$ & $7.4 \times 10^{-13} \exp (700 / T)$ & 8 \\
\hline K158 & $\mathrm{IEPOXOO}+\mathrm{NO}$ & $\rightarrow$ & $\begin{array}{l}0.725 \mathrm{HOCH}_{2} \mathrm{C}(\mathrm{O}) \mathrm{CH}_{3} \\
+0.275 \mathrm{HOCH}_{2} \mathrm{CHO} \\
+0.275 \mathrm{CHOCHO}^{-} \mathrm{CO} \\
+0.275 \mathrm{CH}_{3} \mathrm{C}(\mathrm{O}) \mathrm{CHO}+1.125 \\
\mathrm{OH}+0.825 \mathrm{HO}_{2} \\
+0.2 \mathrm{CO}_{2}+0.375 \mathrm{HCHO}+0.074 \\
\mathrm{HCOOH}+0.251 \mathrm{CO}+\mathrm{NO}_{2}\end{array}$ & $2.7 \times 10^{-12} \exp (360 / T)$ & 3 \\
\hline
\end{tabular}


Table 2. Continued.

\begin{tabular}{|c|c|c|c|c|c|}
\hline No. & Reactants & & Products* & Rate expression & References \\
\hline K159 & $\mathrm{IEPOXOO}+\mathrm{NO}_{3}$ & $\rightarrow$ & $\begin{array}{l}0.725 \mathrm{HOCH}_{2} \mathrm{C}(\mathrm{O}) \mathrm{CH}_{3} \\
+0.275 \mathrm{HOCH}_{2} \mathrm{CHO} \\
+0.275 \mathrm{CHOCHO}^{-} \\
+0.275 \mathrm{CH}_{3} \mathrm{C}(\mathrm{O}) \mathrm{CHO} \\
+1.125 \mathrm{OH}+0.825 \mathrm{HO}_{2} \\
+0.2 \mathrm{CO}_{2}+0.375 \mathrm{HCHO}+0.074 \\
\mathrm{HCOOH}+0.251 \mathrm{CO}+\mathrm{NO}_{2}\end{array}$ & $1.74 \times 2.3 \times 10^{-12}$ & 3 \\
\hline K160 & $\mathrm{MVK}+\mathrm{OH}$ & $\rightarrow$ & MVKOO & $2.6 \times 10^{-12} \exp (610 / T)$ & 1 \\
\hline K161 & $\mathrm{MVK}+\mathrm{NO}_{3}$ & $\rightarrow$ & $\begin{array}{l}0.65 \mathrm{HCOOH} \\
+0.65 \mathrm{CH}_{3} \mathrm{C}(\mathrm{O}) \mathrm{CHO} \\
+0.35 \mathrm{HCHO} \\
+0.35 \mathrm{CH}_{3} \mathrm{C}(\mathrm{O}) \mathrm{COOH}+\mathrm{HNO}_{3}\end{array}$ & $6.0 \times 10^{-16}$ & 1 \\
\hline K162 & $\mathrm{MVK}+\mathrm{O}_{3}$ & $\rightarrow$ & 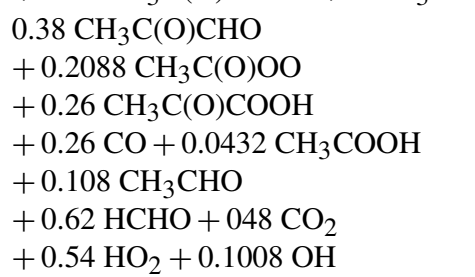 & $8.5 \times 10^{-16} \exp (-1520 / T)$ & 1,3 \\
\hline K163 & $\mathrm{MVKOO}+\mathrm{HO}_{2}$ & $\rightarrow$ & MVKOOH & K144 & \\
\hline K164 & $\mathrm{MVKOO}+\mathrm{NO}$ & $\rightarrow$ & $\begin{array}{l}0.295 \mathrm{CH}_{3} \mathrm{C}(\mathrm{O}) \mathrm{CHO} \\
+0.295 \mathrm{HCHO} \\
+0.670 \mathrm{CH}_{3} \mathrm{CHO} \\
+0.670 \mathrm{HOCH}_{2} \mathrm{CHO} \\
+0.295 \mathrm{HO}_{2} \\
+0.965 \mathrm{NO}_{2} \\
+0.0352 \mathrm{MVKONO}_{2}\end{array}$ & $2.7 \times 10^{-12} \exp (360 / T)$ & 3 \\
\hline K165 & $\mathrm{MVKOOH}+\mathrm{OH}$ & $\rightarrow$ & $\begin{array}{l}\mathrm{CH}_{3} \mathrm{C}(\mathrm{O}) \mathrm{CHO}+\mathrm{CO}+2 \\
\mathrm{HO}_{2}+\mathrm{OH}\end{array}$ & $2.55 \times 10^{-11}$ & 3 \\
\hline K166 & $\mathrm{MVKOOH}+\mathrm{OH}$ & $\rightarrow$ & MVKOO & $1.9 \times 10^{-12} \exp (190 / T)$ & 3 \\
\hline K167 & $\mathrm{MVKONO}_{2}+\mathrm{OH}$ & $\rightarrow$ & $\mathrm{CH}_{3} \mathrm{C}(\mathrm{O}) \mathrm{CHO}+\mathrm{CO}+\mathrm{HO}_{2}+\mathrm{NO}_{2}$ & $1.33 \times 10^{-12}$ & 3 \\
\hline K168 & $\mathrm{MACR}+\mathrm{OH}$ & $\rightarrow$ & MACROO & $8.0 \times 10^{-12} \exp (380 / T)$ & 1 \\
\hline K169 & $\mathrm{MACR}+\mathrm{NO}_{3}$ & $\rightarrow$ & $\mathrm{MACROO}+\mathrm{HNO}_{3}$ & $3.4 \times 10^{-15}$ & 1 \\
\hline K170 & $\mathrm{MACR}+\mathrm{O}_{3}$ & $\rightarrow$ & $\begin{array}{l}0.90 \mathrm{CH}_{3} \mathrm{C}(\mathrm{O}) \mathrm{CHO} \\
+0.5 \mathrm{HCHO}+0.5 \mathrm{CO} \\
+0.14 \mathrm{HO}_{2}+0.24 \mathrm{OH}\end{array}$ & $1.4 \times 10^{-15} \exp (-2100 / T)$ & 1,3 \\
\hline K171 & $\mathrm{MACROO}+\mathrm{HO}_{2}$ & $\rightarrow$ & MACROOH & $0.625 \times 2.91 \times 10^{-13} \exp (1300 / T)$ & 3 \\
\hline K172 & $\mathrm{MACROO}+\mathrm{NO}$ & $\rightarrow$ & $\begin{array}{l}0.987\left(\mathrm{CH}_{3} \mathrm{COCH}_{2} \mathrm{OH}+\mathrm{CO}\right. \\
\left.+\mathrm{NO}_{2}+\mathrm{HO}_{2}\right)+0.013 \\
\mathrm{MACRONO}_{2}\end{array}$ & K164 & 1,3 \\
\hline K173 & $\mathrm{MACROOH}+\mathrm{OH}$ & $\rightarrow$ & $\mathrm{CH}_{3} \mathrm{COCH}_{2} \mathrm{OH}+\mathrm{CO}+\mathrm{OH}$ & $3.77 \times 10^{-11}$ & \\
\hline K174 & $\mathrm{MACROOH}+\mathrm{OH}$ & $\rightarrow$ & MACROO & K166 & \\
\hline K175 & $\mathrm{MACRONO}_{2}+\mathrm{OH}$ & $\rightarrow$ & $\mathrm{CH}_{3} \mathrm{C}(\mathrm{O}) \mathrm{CHO}+\mathrm{CO}+\mathrm{HO}_{2}+\mathrm{NO}_{2}$ & $4.34 \times 10^{-12}$ & 3 \\
\hline K176 & $\mathrm{TERP}+\mathrm{OH}$ & $\rightarrow$ & $\begin{array}{l}0.81 \mathrm{TERPOO}+0.05 \text { ELVOC } \\
+0.14 \mathrm{SVOC}\end{array}$ & $\begin{array}{l}0.5 \times 1.34 \times 10^{-11} \exp (410 / T)+ \\
0.5 \times 1.62 \times 10^{-11} \exp (460 / T)\end{array}$ & 1,10 \\
\hline K177 & $\mathrm{TERP}+\mathrm{NO}_{3}$ & $\rightarrow$ & $2 \mathrm{ISOPONO}_{2}$ & $\begin{array}{l}0.5 \times 1.2 \times 10^{-12} \exp (490 / T)+ \\
0.5 \times 2.5 \times 10^{-12}\end{array}$ & 1,10 \\
\hline K178 & $\mathrm{TERP}+\mathrm{O}_{3}$ & $\rightarrow$ & $\begin{array}{l}0.915 \mathrm{MACR}+0.36 \mathrm{MVK}+0.24 \\
\mathrm{PRV}+1.68 \mathrm{HCHO}+0.16 \mathrm{CO}+ \\
0.6 \mathrm{HCOOH}+0.08 \mathrm{C}_{3} \mathrm{H}_{6}+0.68 \\
\mathrm{OH}+0.05 \mathrm{ELVOC}+0.14 \mathrm{SVOC}\end{array}$ & $\begin{array}{l}0.5 \times 8.22 \times 10^{-16} \exp (-640 / \mathrm{T})+ \\
0.5 \times 1.39 \times 10^{-15} \exp (-1280 / T)\end{array}$ & 1,10 \\
\hline K179 & $\mathrm{TERPOO}+\mathrm{HO}_{2}$ & $\rightarrow$ & 2 ISOPOOH & K144 & \\
\hline K180 & TERPOO + NO & $\rightarrow$ & $\begin{array}{l}2(\mathrm{HCHO}+0.64 \mathrm{MVK} \\
\left.+0.36 \mathrm{MACR}+\mathrm{HO}_{2}\right)+\mathrm{NO}_{2}\end{array}$ & K145 & \\
\hline K181 & $\mathrm{TERPOO}+\mathrm{NO}$ & $\rightarrow$ & $2 \mathrm{ISOPONO}_{2}$ & K146 & \\
\hline
\end{tabular}


Table 2. Continued.

\begin{tabular}{|c|c|c|c|c|c|}
\hline No. & Reactants & & Products* & Rate expression & References \\
\hline K182 & $\mathrm{TERPOO}+\mathrm{NO}_{3}$ & $\rightarrow$ & $\begin{array}{l}2(\mathrm{HCHO}+0.64 \mathrm{MVK} \\
\left.+0.36 \mathrm{MACR}+\mathrm{HO}_{2}\right)+\mathrm{NO}_{2}\end{array}$ & K147 & \\
\hline K183 & $\mathrm{TERPOO}+\mathrm{CH}_{3} \mathrm{OO}$ & $\rightarrow$ & $\begin{array}{l}2(0.64 \mathrm{MVK}+0.36 \mathrm{MACR} \\
\left.+2 \mathrm{HCHO}+2 \mathrm{HO}_{2}\right)\end{array}$ & K148 & \\
\hline K184 & $\mathrm{TERPOO}+\mathrm{CH}_{3} \mathrm{OO}$ & $\rightarrow$ & $\begin{array}{l}2(0.64 \mathrm{MVK}+0.36 \mathrm{MACR} \\
\left.+\mathrm{HCHO}+\mathrm{CH}_{3} \mathrm{OH}\right)\end{array}$ & K149 & \\
\hline K185 & $\mathrm{AROM}+\mathrm{OH}$ & $\rightarrow$ & $\mathrm{AROMOO}+\mathrm{HO}_{2}$ & $\begin{array}{l}\mathrm{A} 1 \times 1.8 \times 10^{-12} \exp (340 / T)+ \\
\mathrm{A} 2 \times 1.72 \times 10^{-11}+ \\
\mathrm{A} 3 \times 2.3 \times 10^{-12} \exp (-190 / T)\end{array}$ & 1,11 \\
\hline K186 & $\mathrm{AROM}+\mathrm{NO}_{3}$ & $\rightarrow$ & $\mathrm{AROMOO}+\mathrm{HNO}_{3}$ & $\begin{array}{l}\mathrm{A} 1 \times 7.8 \times 10^{-17}+ \\
\mathrm{A} 2 \times 3.54 \times 10^{-16}\end{array}$ & 1,11 \\
\hline K187 & $\mathrm{AROM}+\mathrm{O}_{3}$ & $\rightarrow$ & AROMOO & $\begin{array}{l}\mathrm{A} 1 \times 1.0 \times 10^{-21}+ \\
\mathrm{A} 2 \times\left(2.4 \times 10^{-13} \exp (-5586 / T)\right. \\
+5.37 \times 10^{-13} \exp (-6039 / T) \\
\left.+1.91 \times 10^{-13} \exp (-5586 / T)\right) / 3\end{array}$ & $1,11,12$ \\
\hline K188 & $\mathrm{AROMOO}+\mathrm{HO}_{2}$ & $\rightarrow$ & $\mathrm{C}_{4} \mathrm{H}_{9} \mathrm{OOH}+\mathrm{CHOCHO}+\mathrm{HCHO}$ & K126 & \\
\hline K189 & $\mathrm{AROMOO}+\mathrm{NO}$ & $\rightarrow$ & $\begin{array}{l}\mathrm{NO}_{2}+0.67 \mathrm{CH}_{3} \mathrm{CH}_{2} \mathrm{COCH}_{3} \\
+0.67 \mathrm{HO}_{2}+0.33 \mathrm{C}_{2} \mathrm{H}_{5} \mathrm{OO} \\
+0.33 \mathrm{CH}_{3} \mathrm{CHO}+\mathrm{CHOCHO} \\
+\mathrm{HCHO}\end{array}$ & K127 & \\
\hline K190 & $\mathrm{AROMOO}+\mathrm{NO}$ & $\rightarrow$ & $\mathrm{C}_{4} \mathrm{H}_{9} \mathrm{ONO}_{2}+\mathrm{CHOCHO}+\mathrm{HCHO}$ & K128 & \\
\hline K191 & $\mathrm{AROMOO}+\mathrm{CH}_{3} \mathrm{OO}$ & $\rightarrow$ & $\begin{array}{l}\mathrm{HCHO}+\mathrm{HO}_{2} \\
+0.67\left(\mathrm{CH}_{3} \mathrm{CH}_{2} \mathrm{C}(\mathrm{O}) \mathrm{CH}_{3}+\mathrm{HO}_{2}\right) \\
+0.33\left(\mathrm{CH}_{3} \mathrm{CHO}+\mathrm{CH}_{3} \mathrm{CH}_{2} \mathrm{OO}\right) \\
+\mathrm{CHOCHO}+\mathrm{HCHO}\end{array}$ & K129 & \\
\hline K192 & $\mathrm{AROMOO}+\mathrm{CH}_{3} \mathrm{OO}$ & $\rightarrow$ & $\begin{array}{l}\mathrm{CH}_{3} \mathrm{CH}_{2} \mathrm{COCH}_{3}+\mathrm{CH}_{3} \mathrm{OH} \\
+\mathrm{CHOCHO}+\mathrm{HCHO}\end{array}$ & K130 & \\
\hline K193 & $\mathrm{SO}_{2}+\mathrm{OH}$ & $\rightarrow$ & $\mathrm{HO}_{2}+\mathrm{H}_{2} \mathrm{SO}_{4}$ & $\begin{array}{l}3.3 \times 10^{-31}(T / 300)^{-4.3}\left[\mathrm{~N}_{2}\right] \\
1.6 \times 10^{-12}(T / 300)^{-0.7} \\
\mathrm{Fc}=0.6\end{array}$ & 2 \\
\hline K194 & $\mathrm{DMS}+\mathrm{OH}$ & $\rightarrow$ & $\mathrm{CH}_{3} \mathrm{OO}+\mathrm{HCHO}+\mathrm{SO}_{2}$ & $1.1 \times 10^{-11} \exp (-240 / T)$ & 2 \\
\hline K195 & $\mathrm{DMS}+\mathrm{OH}$ & $\rightarrow$ & $\begin{array}{l}0.75 \mathrm{CH}_{3} \mathrm{OO}+0.75 \mathrm{HCHO}+0.75 \\
\mathrm{SO}_{2}+0.25 \mathrm{MSA}\end{array}$ & $\begin{array}{l}1.0 \times 10^{-39}\left[\mathrm{O}_{2}\right] \exp (5820 / T) / \\
\left(1+5.0 \times 10^{-30}\left[\mathrm{O}_{2}\right] \exp (6280 / T)\right)\end{array}$ & 2 \\
\hline K196 & $\mathrm{DMS}+\mathrm{NO}_{3}$ & $\rightarrow$ & $\mathrm{CH}_{3} \mathrm{OO}+\mathrm{HCHO}+\mathrm{SO}_{2}+\mathrm{HNO}_{3}$ & $1.9 \times 10^{-13} \exp (520 / T)$ & 2 \\
\hline K197 & $\mathrm{NH}_{3}+\mathrm{OH}$ & $\rightarrow$ & $\mathrm{NH}_{2}+\mathrm{HO}_{2}$ & $1.7 \times 10^{-12} \exp (-710 / T)$ & 2 \\
\hline K198 & $\mathrm{NH}_{2}+\mathrm{O}_{2}$ & $\rightarrow$ & $\mathrm{NH}_{2} \mathrm{O}_{2}$ & $6.0 \times 10^{-21}$ & 2 \\
\hline K199 & $\mathrm{NH}_{2}+\mathrm{O}_{3}$ & $\rightarrow$ & $\mathrm{NH}_{2} \mathrm{O}_{2}$ & $4.3 \times 10^{-12} \exp (-930 / T)$ & 2 \\
\hline $\mathrm{K} 200$ & $\mathrm{NH}_{2}+\mathrm{OH}$ & $\rightarrow$ & $\mathrm{NH}_{2} \mathrm{O}_{2}$ & $3.4 \times 10^{-11}$ & 2 \\
\hline K201 & $\mathrm{NH}_{2}+\mathrm{HO}_{2}$ & $\rightarrow$ & $\mathrm{NH}_{3}$ & $3.4 \times 10^{-11}$ & 2 \\
\hline K202 & $\mathrm{NH}_{2}+\mathrm{NO}$ & $\rightarrow$ & $\mathrm{NH}_{2} \mathrm{O}_{2}+\mathrm{NO}_{2}$ & $4.0 \times 10^{-12} \exp (450 / T)$ & 2 \\
\hline K203 & $\mathrm{NH}_{2}+\mathrm{NO}_{2}$ & $\rightarrow$ & $\mathrm{NH}_{2} \mathrm{O}_{2}+\mathrm{NO}$ & $2.1 \times 10^{-12} \exp (650 / T)$ & 2 \\
\hline K204 & $\mathrm{NH}_{2} \mathrm{O}_{2}+\mathrm{O}_{3}$ & $\rightarrow$ & $\mathrm{NH}_{2}$ & K199 & \\
\hline K205 & $\mathrm{NH}_{2} \mathrm{O}_{2}+\mathrm{HO}_{2}$ & $\rightarrow$ & $\mathrm{NH}_{2}$ & K201 & \\
\hline K206 & $\mathrm{NH}_{2} \mathrm{O}_{2}+\mathrm{NO}$ & $\rightarrow$ & $\mathrm{NH}_{2}+\mathrm{NO}_{2}$ & K202 & \\
\hline
\end{tabular}

* The reaction products $\mathrm{O}_{2}, \mathrm{H}_{2}$, and $\mathrm{H}_{2} \mathrm{O}$ are not shown.

1 The chemical kinetic data and mechanistic information were taken from the website of the IUPAC Task Group on Atmospheric Chemical Kinetic Data Evaluation: http://iupac.pole-ether.fr/ (last access: 20 August 2019).

2 The chemical kinetic data and mechanistic information were taken from the website of the NASA Panel for Data Evaluation (Evaluation No. 18, JPL Publication 15-10; http://jpldataeval.jpl.nasa.gov, last access: 20 August 2019).

3 The chemistry mechanistic information was taken from the Master Chemical Mechanism (MCM v3.3.1) - for nonaromatic schemes: Jenkin et al. (1997); Saunders et al. (2003); for the isoprene scheme: Jenkin et al. (2015); for aromatic schemes: Jenkin et al. (2003); Bloss et al. (2005); via the website http://mcm.leeds.ac.uk/MCM (last access: 20 August 2019).

4 Atkinson (1997): $R_{1}=2.7 \times 10^{14} \exp (-6350 / T) ; R_{2}=6.3 \times 10^{-14} \exp (-550 / T) ; f=R_{1} /\left(R_{1}+R_{2} \times\left[\mathrm{O}_{2}\right]\right) ; R_{1}=1.94 \times 10^{-22}[\mathrm{AIR}] \exp \left(0.972 \times N_{\mathrm{c}}\right)$;

$R_{2}=0.826 \times(T / 300)^{-8.1} ; A=1 /\left(1+\log _{10}\left(R_{1} / R_{2}\right)^{2}\right) ; \operatorname{RTC}\left(N_{\mathrm{c}}\right) P=0.4 \times R_{1} /\left(1+R_{1} / R_{2}\right) 0.411^{A} ; \operatorname{RTC}\left(N_{\mathrm{c}}\right) S=R 1 /\left(1+R_{1} / R_{2}\right) 0.411^{A}$,

where $N_{\mathrm{c}}$ is the number of carbons (i.e., $\left.1-5\right)$.

${ }^{5}$ Orlando et al. (1992); Poisson et al. (2000); ${ }^{6}$ Peeters and Müller (2010); ${ }^{7}$ Crounse et al. (2011); ${ }^{8}$ Paulot et al. (2009); ${ }^{9}$ Browne et al. (2014); ${ }^{10}$ Average of $\alpha$ - and

$\beta$-pinene; ${ }^{11} \mathrm{~A} 1, \mathrm{~A} 2$, and $\mathrm{A} 3$ represent the relative contributions of ortho-, meta-, and para-xylene (A1), toluene (A2), and benzene (A3); roughly $0.4,0.6$, and 0.4 ,

respectively, for the year 2006 .

12 Average of ortho-, meta-, and para-isomers of xylene. 
latitude $\left(60-90^{\circ} \mathrm{S}-\mathrm{N}\right)$ forest fires (see the Appendix in van Noije et al., 2014).

Biogenic emissions from vegetation include isoprene, terpenes and other volatile organic compounds, and CO. Emissions are based on the Model of Emissions of Gases and Aerosols from Nature (MEGAN) version 2.1 (Sindelarova et al., 2014). Isoprene and terpene emissions are distributed over the first $\sim 50 \mathrm{~m}$ from the surface and a diurnal cycle is imposed. The biogenic emissions from soils include $\mathrm{NO}_{x}$ (Yienger and Levy, 1995), $\mathrm{NH}_{3}$ and terrestrial DMS emissions from soils and vegetation (Spiro et al., 1992). Oceanic emissions of $\mathrm{CO}$ and NMVOCs come from the POET database (Granier et al., 2005), oceanic emissions of $\mathrm{NH}_{3}$ from Bouwman et al. (1997), and the DMS oceanic emissions are calculated online using the seawater concentration climatology from Lana et al. (2011). The $\mathrm{NO}_{x}$ production by lightning is parameterized based on convective precipitation fields (Meijer et al., 2001), and the $\mathrm{SO}_{x}$ fluxes from continuously emitting volcanoes are taken from Andres and Kasgnoc (1998). Note that we focus below on the more detailed representation of emissions as used for the MOGUNTIA chemical scheme. Emissions of other tropospheric species in the gas and the particulate phase are described in detail in previous studies (e.g., van Noije et al., 2014).

The MOGUNTIA chemical scheme considers direct emissions of $\mathrm{CO}, \mathrm{CH}_{4}, \mathrm{HCHO}, \mathrm{HCOOH}, \mathrm{CH}_{3} \mathrm{OH}, \mathrm{C}_{2} \mathrm{H}_{6}, \mathrm{C}_{2} \mathrm{H}_{4}$, $\mathrm{C}_{2} \mathrm{H}_{2}, \mathrm{CH}_{3} \mathrm{CHO}, \mathrm{CH}_{3} \mathrm{COOH}, \mathrm{C}_{2} \mathrm{H}_{5} \mathrm{OH}, \mathrm{HOCH}_{2} \mathrm{CHO}$, $\mathrm{CHOCHO}, \mathrm{C}_{3} \mathrm{H}_{8}, \mathrm{C}_{3} \mathrm{H}_{6}, n-\mathrm{C}_{4} \mathrm{H}_{10}, \mathrm{MEK}, \mathrm{C}_{5} \mathrm{H}_{8}, \mathrm{C}_{10} \mathrm{H}_{16}$, and $\mathrm{C}_{7} \mathrm{H}_{8}$, as well as $\mathrm{NO}_{x}, \mathrm{NH}_{3}$, DMS, and $\mathrm{SO}_{x}$. Butanes, pentanes, hexanes, and higher alkane emissions are summed up into the lumped $n-\mathrm{C}_{4} \mathrm{H}_{10}$ species, which represents the alkanes containing four or more carbon atoms. For reactivity purposes, higher alkene emissions containing four or more carbon atoms (butenes and higher alkenes) are accounted for as equivalent $\mathrm{C}_{3} \mathrm{H}_{6}$ emissions. Higher ketones (i.e., except for acetone) from open biomass burning emissions are represented as MEK. Emissions of benzene $\left(\mathrm{C}_{6} \mathrm{H}_{6}\right)$, toluene $\left(\mathrm{C}_{7} \mathrm{H}_{8}\right)$, xylene $\left(\mathrm{C}_{8} \mathrm{H}_{10}\right)$, trimethyl-benzenes, and other higher aromatics and VOCs are represented by toluene as in the MOZART mechanism (Emmons et al., 2010). Note that when VOC emissions are assigned to a lumped species, adjustments are made to preserve their atmospheric reactivity (see also notes in Tables 1 and 2).

The explicit parameterization of VOC species in the MOGUNTIA chemical scheme requires emissions that are not routinely included in available emission databases. Direct biofuel and biomass burning emissions of light carbonyls have been reported in several studies (e.g., Christian et al., 2003; Fu et al., 2008; Hays et al., 2002), and these represent a significant contribution to the VOC budget (e.g., Fu et al., 2008; Myriokefalitakis et al., 2008; Stavrakou et al., 2009b, a; Vrekoussis et al., 2009). For this reason, emissions from biofuel use of 1.4, 2.4, and $1.6 \mathrm{Tg} \mathrm{yr}^{-1}$ are considered for GLYAL, GLY, and MGLY, respectively. For the biomass burning sector, we use global emissions of GLYAL and GLY of 4.3 and $5.2 \mathrm{Tg} \mathrm{yr}^{-1}$, respectively. We base these emission rates on the $\mathrm{HCHO}$ emissions distribution because mass emission rates of low-molecular-weight carbonyls, such as HCHO and GLY (e.g., Hays et al., 2002), are highly correlated. Global emissions of roughly $1.4 \mathrm{Tg} \mathrm{yr}^{-1}$ (Emmons et al., 2010) are also considered for MEK, accounting for anthropogenic emissions (Rodigast et al., 2016) such as domestic burning and solvent use (e.g., Ware, 1988). For all other carbonyls, primary anthropogenic emissions are considered negligible (e.g., Fu et al., 2008). A list of the global annual emission strengths considered for the MOGUNTIA chemical configuration is presented in Table 3. For completeness, we note that primary aerosol emissions of $\mathrm{OC}, \mathrm{BC}$, sea salt, and dust are also considered in the model, with sea salt and dust emissions calculated online. A more detailed description of the gas and aerosol emissions used in the model will be presented in van Noije et al. (2020).

\subsection{Simulations}

We will present the analysis of TM5-MP simulations with the mCB05 and MOGUNTIA chemical mechanisms for the year 2006, which has been the chosen year of previous benchmarking studies (Huijnen et al., 2010; Williams et al., 2013, 2017). All simulations have been performed at $1^{\circ} \times 1^{\circ}$ horizontal resolution (e.g., Williams et al., 2017) with 34 vertical layers and use a 1-year spin-up (i.e., for the year 2005). The same emission datasets have been used in all simulations, albeit with higher speciation for the MOGUNTIA chemical scheme. Overall, two simulations have been performed for the mCB05 configuration: one employing the EBI solver (mCB05(EBI)) and one employing the KPP-generated Rosenbrock solver (mCB05(KPP)). This approach isolates differences that are caused solely by the applied chemistry solver. By comparing MOGUNTIA generated by KPP with $\mathrm{mCB} 05(\mathrm{KPP})$, the differences due to the chemistry setup in the model are isolated.

\section{Model performance}

Concerning the TM5-MP performance, simulations performed on the ECMWF CRAY XC40 high-performance computer facility using 360 cores indicate that the coupling of KPP software alone increases the time spent in chemistry by $\sim 59 \%$ and overall slows down the code by $\sim 18 \%$ compared to the (hand-coded) EBI version for the mCB05 mechanism. As expected, the coupling of the MOGUNTIA atmospheric chemistry scheme further increases the model runtime. MOGUNTIA uses 100 transported and 28 nontransported tracers, numbers that are significantly larger than the mCB05 configuration (i.e., 69 transported and 21 nontransported tracers). As a result, time spent to transport the tracers increases by $\sim 43 \%$, and the chemistry calculations slow down by $\sim 55 \%$. Altogether, the newly coupled MO- 
Table 3. Global annual emissions of trace gases used for the MOGUNTIA chemistry scheme in TM5-MP for the year 2006 (Tg yr ${ }^{-1}$ unless $^{-}$ specified otherwise).

\begin{tabular}{|c|c|c|c|c|c|c|c|c|}
\hline \multirow[t]{2}{*}{ Species } & \multirow[t]{2}{*}{ Long name } & \multicolumn{7}{|c|}{ Emissions } \\
\hline & & Anthropogenic ${ }^{\mathrm{a}}$ & Biomass burning & Biogenic & Soil & Oceanic & Other & Total \\
\hline $\mathrm{CO}$ & carbon monoxide & 600.5 & 386.4 & 90.2 & & 19.9 & & 1097 \\
\hline $\mathrm{HCHO}$ & formaldehyde & 2.4 & 5.2 & 4.7 & & & & 12.3 \\
\hline $\mathrm{HCOOH}$ & formic acid & 4.6 & 1.8 & 3.5 & & & & 9.8 \\
\hline $\mathrm{CH}_{3} \mathrm{OH}$ & methanol & 4.7 & 9.8 & 131.9 & & & & 146.4 \\
\hline $\mathrm{C}_{2} \mathrm{H}_{6}$ & ethane & 6.2 & 3.4 & 0.3 & & 1.0 & & 10.9 \\
\hline $\mathrm{C}_{2} \mathrm{H}_{4}$ & ethene & 5.3 & 4.8 & 18.3 & & 1.4 & & 29.8 \\
\hline $\mathrm{C}_{2} \mathrm{H}_{2}$ & acetylene & 3.3 & & & & & & 3.3 \\
\hline $\mathrm{CH}_{3} \mathrm{CHO}$ & acetaldehyde & 1.2 & 4.4 & 21.9 & & & & 27.5 \\
\hline $\mathrm{CH}_{3} \mathrm{COOH}$ & acetic acid & 4.6 & 18.0 & 3.5 & & & & 26.1 \\
\hline $\mathrm{CH}_{3} \mathrm{CH}_{2} \mathrm{OH}$ & ethanol & 0.5 & 0.1 & 18.6 & & & & 19.3 \\
\hline $\mathrm{HOCH}_{2} \mathrm{CHO}$ & glycol-aldehyde & 1.4 & 4.3 & & & & & 5.7 \\
\hline CHOCHO & glyoxal & 2.4 & 5.2 & & & & & 7.6 \\
\hline $\mathrm{C}_{3} \mathrm{H}_{8}$ & propane & 6.5 & 0.7 & 0.03 & & 1.3 & & 8.5 \\
\hline $\mathrm{C}_{3} \mathrm{H}_{6}$ & $\begin{array}{l}\text { propene and higher } \\
\text { alkenes }\end{array}$ & 8.3 & 4.8 & 17.5 & & 1.5 & & 32.1 \\
\hline $\mathrm{CH}_{3} \mathrm{COCH}_{3}$ & acetone & 2.7 & 1.7 & 37.7 & & & & 42.1 \\
\hline $\mathrm{CH}_{3} \mathrm{C}(\mathrm{O}) \mathrm{CHO}$ & methylglyoxal & 1.6 & 3.4 & & & & & 5.0 \\
\hline $\mathrm{C}_{4} \mathrm{H}_{10}$ & $\begin{array}{l}\text { butane and higher } \\
\text { alkanes (including bu- } \\
\text { tane, pentane, hexane, } \\
\text { higher alkanes, and } \\
\text { other VOCs) }\end{array}$ & 52.8 & 0.5 & 0.1 & & & & 53.4 \\
\hline $\mathrm{CH}_{3} \mathrm{CH}_{2} \mathrm{COCH}_{3}$ & $\begin{array}{l}\text { methyl-ethyl-ketone } \\
\text { (including higher } \\
\text { ketones except for } \\
\text { acetone) }\end{array}$ & 1.4 & 1.4 & 0.9 & & & & 3.7 \\
\hline $\mathrm{C}_{5} \mathrm{H}_{8}$ & isoprene & & & 579.4 & & & & 579.4 \\
\hline $\mathrm{C}_{10} \mathrm{H}_{16}$ & monoterpenes & & & 97.9 & & & & 97.9 \\
\hline $\mathrm{C}_{7} \mathrm{H}_{8}$ & $\begin{array}{l}\text { toluene and aromatics } \\
\text { (including toluene, } \\
\text { xylene benzene, } \\
\text { trimethylbenzene, and } \\
\text { higher aromatics) }\end{array}$ & 25.3 & 4.0 & 1.5 & & & & 30.8 \\
\hline $\mathrm{NO}_{x}{ }^{\mathrm{b}}$ & nitrogen oxides & 42.3 & 6.6 & & 5.0 & & $6.0^{\mathrm{c}}$ & 59.9 \\
\hline $\mathrm{NH}_{3}$ & ammonia & 56.1 & 4.4 & & 2.3 & 8.1 & & 70.9 \\
\hline $\mathrm{SO}_{2}$ & sulfur dioxide & 120.5 & 2.3 & & & & $9.3^{\mathrm{d}}$ & 132.1 \\
\hline $\mathrm{CH}_{3} \mathrm{SCH}_{3}$ & dimethylsulfide & & & 1.7 & & 95.8 & & 97.5 \\
\hline
\end{tabular}

${ }^{\mathrm{a}}$ Including aircraft emissions; ${ }^{\mathrm{b}} \mathrm{Tg} \mathrm{N} \mathrm{yr}-1 ;{ }^{\mathrm{c}} \mathrm{NO}_{x}$ production from lightning; ${ }^{\mathrm{d}} \mathrm{SO}_{2}$ from volcanoes.

GUNTIA chemistry scheme in TM5-MP is computationally $\sim 27 \%$ more expensive than the mCB05(EBI) configuration. Overall, the mCB05(EBI), mCB05(KPP), and MOGUNTIA configurations simulate $0.73,0.60$, and 0.44 years per day of simulation time, respectively (Table S3a). Note that an additional series of simulations with 450 cores leads to only marginal changes (Table S3b). Finally, the runtime values for the different model configurations presented here are highly hardware-dependent, owing mainly to the large I/O component associated with reading the meteorological fields.

\section{Comparison of budgets and tropospheric mixing ratios}

\subsection{Ozone $\left(\mathrm{O}_{3}\right)$}

Table 4 presents a detailed description of the chemical budget of tropospheric ozone, as calculated by the TM5-MP model, for the three chemical configurations. Following Stevenson et al. (2006), chemical production of ozone is derived from all reactions that convert $\mathrm{NO}$ to $\mathrm{NO}_{2}$, since $\mathrm{NO}_{2}$ is rapidly 
photodissociated and forms $\mathrm{O}_{3}$, i.e.,

$\mathrm{NO}+\mathrm{HO}_{2} \rightarrow \mathrm{NO}_{2}+\mathrm{OH}$,

$\mathrm{NO}+\mathrm{RO}_{2} \rightarrow \mathrm{NO}_{2}+\mathrm{RO}$,

where $\mathrm{RO}_{2}$ represents all the major organic peroxy radicals of the corresponding chemistry mechanism used in the model. For the MOGUNTIA scheme $\mathrm{RO}_{2}$ includes $\mathrm{CH}_{3} \mathrm{O}_{2}$, $\mathrm{C}_{2} \mathrm{H}_{5} \mathrm{O}_{2}, \mathrm{HYEO}_{2}, \mathrm{n}-\mathrm{C}_{3} \mathrm{H}_{7} \mathrm{O}_{2}, \mathrm{i}-\mathrm{C}_{3} \mathrm{H}_{7} \mathrm{O}_{2}, \mathrm{ACO}_{2}, \mathrm{HYPO}_{2}$, n$\mathrm{C}_{4} \mathrm{H}{ }_{9} \mathrm{O}, \mathrm{MEKO}_{2}, \mathrm{ISOPO}_{2}, \mathrm{IEPOXO}_{2}, \mathrm{MVKO}_{2}, \mathrm{MACRO}_{2}$, $\mathrm{TERO}_{2}$, and $\mathrm{AROO}_{2}$ radicals. For $\mathrm{mCB} 05, \mathrm{RO}_{2}$ includes the $\mathrm{CH}_{3} \mathrm{O}_{2}$ radical and $\mathrm{XO}_{2}$ (i.e., the operator for the $\mathrm{NO}$ to $\mathrm{NO}_{2}$ conversion, which represents all lumped alkyl-peroxy radicals in mCB05; see Williams et al., 2017, and Yarwood et al., 2005).

The chemical $\mathrm{O}_{3}$ loss is derived as the sum of the

1. $\mathrm{O}_{3}$ photolysis to $\mathrm{O}\left({ }^{1} \mathrm{D}\right)$, i.e.,

$$
\mathrm{O}_{3}+h v \rightarrow \mathrm{O}\left({ }^{1} \mathrm{D}\right)+\mathrm{O}_{2},
$$

followed by reaction with $\mathrm{H}_{2} \mathrm{O}$ to form $\mathrm{OH}$, i.e.,

$$
\mathrm{O}\left({ }^{1} \mathrm{D}\right)+\mathrm{H}_{2} \mathrm{O} \rightarrow 2 \mathrm{OH},
$$

2. $\mathrm{O}_{3}$ destruction by $\mathrm{HO}_{2}$ and $\mathrm{OH}$ catalytic cycles, i.e.,

$$
\begin{aligned}
& \mathrm{O}_{3}+\mathrm{HO}_{2} \rightarrow \mathrm{OH}+2 \mathrm{O}_{2}, \\
& \mathrm{O}_{3}+\mathrm{OH} \rightarrow \mathrm{HO}_{2}+\mathrm{O}_{2},
\end{aligned}
$$

and

3. reactions of $\mathrm{O}_{3}$ with unsaturated VOCs. Chemical loss calculations exclude contributions from $\mathrm{HNO}_{3}, \mathrm{NO}_{3}$, $\mathrm{N}_{2} \mathrm{O}_{5}$, and other fast cycles between ozone-related species, as proposed by Stevenson et al. (2006).

For the MOGUNTIA scheme, the tropospheric chemical production is calculated to be $5709 \mathrm{Tg} \mathrm{yr}^{-1}$, which is only $\sim 10 \mathrm{Tg} \mathrm{yr}^{-1}$ smaller compared to the mCB05(KPP) configuration. Chemical destruction in the troposphere is similar in the MOGUNTIA and mCB05(KPP) chemistry configurations (Table 4). The use of EBI compared to the Rosenbrock solver decreases the $\mathrm{O}_{3}$ chemical production (5719 vs. $5589 \mathrm{Tg} \mathrm{yr}^{-1}$ ) and destruction (5216 vs. $5192 \mathrm{Tg} \mathrm{yr}^{-1}$ ) terms in the troposphere (Table 4). Besides some expected differences due to the behavior of the two solvers, the calculated differences may also be partly attributed to the mass fixer for $\mathrm{NO}_{Y}$ (i.e., the sum of $\mathrm{NO}, \mathrm{NO}_{2}, \mathrm{NO}_{3}, \mathrm{HNO}_{3}, \mathrm{HNO}_{4}$, $2 \times \mathrm{N}_{2} \mathrm{O}_{5}$, PAN, and the organic nitrate compounds) that is applied in the mCB05(EBI) configuration to ensure no artificial loss of nitrogen. $\mathrm{NO}_{Y}$ fixing occurs mainly over highly polluted regions with active $\mathrm{NO}_{x}$ photochemistry to improve the accuracy of the EBI solver.

Focusing on the impact of the stratosphere on the tropospheric $\mathrm{O}_{3}$ budget, the net STE flux of $\mathrm{O}_{3}$ for the MOGUNTIA configuration is somewhat lower $(\sim 1 \%)$ than for
mCB05(KPP). Considering that all configurations use the same stratospheric ozone relaxation parameterization, this difference can only be attributed to the chemical schemes. Note that the global STE of $\mathrm{O}_{3}$ is defined by simply considering the chemical production and loss budget terms, as proposed by Stevenson et al. (2006). The differences in the $\mathrm{O}_{3}$ stratospheric inflow budgets for the three chemistry configurations (Table 4) do not imply that the tropospheric chemistry impacts $\mathrm{O}_{3}$ transport from the stratosphere, but rather that the global budget is closed by an inferred stratospheric input term. Thus, the higher net chemical production of $\mathrm{O}_{3}$ in the troposphere implies a lower contribution from the stratosphere to the troposphere for roughly the same deposition losses. The calculated net influx from the stratosphere for the MOGUNTIA configuration $\left(\sim 424 \mathrm{Tg} \mathrm{yr}^{-1}\right)$ remains within 1 standard deviation of a multi-model mean (552 \pm $168 \mathrm{Tg} \mathrm{yr}^{-1}$ ), as reported by both Stevenson et al. (2006) and Young et al. (2013). MOGUNTIA calculations are also in line with estimates $\left(\sim 400 \mathrm{Tg} \mathrm{yr}^{-1}\right)$ based on observations (Hsu, 2005; Olsen, 2004), although they are higher compared to the $306 \mathrm{Tg} \mathrm{yr}^{-1}$ calculated by an earlier version of the TM5 model driven by the same meteorological fields (van Noije et al., 2014). Overall, compared to the mCB05(EBI) simulation, the lower net stratosphere-troposphere exchange flux simulated in the MOGUNTIA configuration brings the model results closer to the current best estimates of the net STE.

The MOGUNTIA configuration also results in a reduction of roughly $2 \%$ in the tropospheric $\mathrm{O}_{3}$ burden compared to both mCB05 configurations. No significant change in the $\mathrm{O}_{3}$ lifetime in the troposphere (i.e., 22.3-22.8 d) is found, and the calculated lifetimes remain close to other model estimates of 22 d (Stevenson et al., 2006; Young et al., 2013). Compared to previous studies, the tropospheric $\mathrm{O}_{3}$ burden calculated using the MOGUNTIA chemical configuration $(\sim 375$ $\mathrm{Tg}$ ) is $\sim 12 \%$ higher compared to the multi-model mean estimate of Stevenson et al. (2006) $(336 \pm 27 \mathrm{Tg})$ and the $335 \pm 10 \mathrm{Tg}$ burden derived from $\mathrm{O}_{3}$ climatology from pre2000 data (Wild, 2007), as well as $\sim 20 \%$ higher compared to the tropospheric burden of $309 \mathrm{Tg}$ reported by van Noije et al. (2014). The calculated burden for the MOGUNTIA chemistry configuration is also $\sim 11 \%$ higher compared to the burden derived from the ACCMIP models (337 $\pm 23 \mathrm{Tg}$; Young et al. 2013), roughly $17 \%$ higher than the burden reported by Schultz et al. (2018), and 8\%-15\% higher than the Lamarque et al. (2012) estimations, who used a tropopause level at $100 \mathrm{ppb}$ of $\mathrm{O}_{3}$ mixing ratios. Table 4 also presents the relative differences of the budget calculations when a tropopause level of $100 \mathrm{ppb} \mathrm{O}_{3}$ is adopted. Note that the tropospheric burden estimates remain susceptible to the tropopause definition, leading potentially to significant differences between modeling studies. For this reason, the tropopause level(s) should always be reported when comparing modeling estimates. Overall, the use of the MOGUNTIA mechanism tends to bring the model closer to other published estimates by 
Table 4. Tropospheric budgets and burden $\left(\mathrm{Tg}\left(\mathrm{O}_{3}\right)\right)$ of $\mathrm{O}_{3}$ for the year $2006\left(\mathrm{Tg}\left(\mathrm{O}_{3}\right) \mathrm{yr}^{-1}\right)$ using the 150 ppb $\mathrm{O}_{3}$ mixing ratio to define the tropopause level. In parentheses, the relative differences using the $100 \mathrm{ppb} \mathrm{O}_{3}$ mixing ratios are also presented, calculated with reference to the $150 \mathrm{ppb} \mathrm{O}_{3}$ tropopause level definition.

\begin{tabular}{|c|c|c|c|c|c|c|c|c|c|c|c|c|c|}
\hline \multirow{2}{*}{$\begin{array}{l}\text { Production terms } \\
\text { Stratospheric inflow* }\end{array}$} & \multicolumn{2}{|c|}{ mCB05 (EBI) } & \multicolumn{2}{|c|}{ mCB05 (KPP) } & \multicolumn{2}{|c|}{ MOGUNTIA } & \multirow{2}{*}{$\begin{array}{l}\text { Loss terms } \\
\text { Deposition }\end{array}$} & \multicolumn{2}{|c|}{ mCB05 (EBI) } & \multicolumn{2}{|c|}{ mCB05 (KPP) } & \multicolumn{2}{|c|}{ MOGUNTIA } \\
\hline & 632 & $(10 \%)$ & 429 & $(32 \%)$ & 424 & $(30 \%)$ & & 955 & $(0 \%)$ & 932 & $(0 \%)$ & 913 & $(0 \%)$ \\
\hline Trop. chem. production & 5589 & $(-3 \%)$ & 5719 & $(-3 \%)$ & 5709 & $(-3 \%)$ & Trop. chem. loss & 5192 & $(-1 \%)$ & 5216 & $(-1)$ & 5219 & $(-1 \%)$ \\
\hline Trop. burden & 385 & $(-8 \%)$ & 384 & $(-8 \%)$ & 375 & $(-8 \%)$ & Trop. lifetime (days) & 22.8 & $(-8 \%)$ & 22.8 & $(-8 \%)$ & 22.3 & $(-6 \%)$ \\
\hline
\end{tabular}

* Sum of the deposition and the tropospheric chemical loss minus the production.

lowering the $\mathrm{O}_{3}$ burden compared to the mCB05 scheme in TM5-MP.

Ozone surface and zonal mean mixing ratios simulated by the MOGUNTIA configuration for the year 2006 are presented in Fig. 1a and b, respectively. Figure 1c and d show small differences in surface and zonal mean mixing ratios between MOGUNTIA and mCB05(KPP). Differences in surface simulated $\mathrm{O}_{3}$ mixing ratios between the two mechanisms are evident mainly downwind of regions with biogenic and tropical fire emissions. The mCB05(KPP) simulation shows higher mixing ratios $(\sim 2-4 \mathrm{ppb})$ over the Intertropical Convergence Zone (ITCZ), India, and East Asia (up to $\sim 10 \mathrm{ppb}$ ). This is mainly attributed to the different representation of VOCs, with MOGUNTIA being significantly more explicit than mCB05. This behavior can also be observed in the zonal mean $\mathrm{O}_{3}$ distribution presented in Fig. 1d, where the impact of the different representation of VOCs, originating mainly from the tropics, is reaching the middle and upper troposphere lifted by convection following the upward branch of the tropical Hadley cell. The use of different solvers alone does not result in any critical difference in the $\mathrm{O}_{3}$ mixing ratios for mCB05 (Fig. 1e, f), presenting only some small negative differences of $\sim 1 \mathrm{ppb}$ downwind of regions with high anthropogenic emissions (e.g., India) for $\mathrm{mCB} 05(\mathrm{EBI})$.

\subsection{Hydroxyl radical $(\mathrm{OH})$}

The hydroxyl radical $(\mathrm{OH})$ is the primary oxidant in the atmosphere under sunlit conditions, initiating the oxidation of various VOCs and thus the production of hydroperoxy $\left(\mathrm{HO}_{2}\right)$ and organic peroxy $\left(\mathrm{RO}_{2}\right)$ radicals. However, due to the high complexity of $\mathrm{OH}$ recycling pathways in atmospheric VOC degradation, the different representations of VOC oxidation pathways in chemical mechanisms may lead to significant discrepancies between models. $\mathrm{CH}_{4}$ is routinely used as a diagnostic for the calculated $\mathrm{OH}$ abundance in the troposphere since its background concentration is highly sensitive to the $\mathrm{OH}$ abundance in the tropics, where water vapor and biogenic emissions are high. Uncertainties in $\mathrm{CH}_{4}$ global sources (e.g., a rapid rise in the $\mathrm{CH}_{4}$ growth rates since 2007; Nisbet et al., 2019), together with uncertainties in anthropogenic emissions of $\mathrm{NO}_{x}, \mathrm{CO}$, and NMVOCs (e.g., Hoesly et al., 2018), may cause considerable divergence in model-simulated $\mathrm{CH}_{4}$ mixing ratios for different simulation years. For the present study, however, the surface mixing ratios of $\mathrm{CH}_{4}$ are prescribed according to the CMIP6 recommendations for each simulation year.

Table 5 presents the global tropospheric $\mathrm{OH}$ production budgets for the various chemical configurations. The MOGUNTIA configuration yields a gas-phase $\mathrm{OH}$ formation via $\mathrm{O}_{3}$ photolysis in the presence of water molecules (Reactions R3 and R4) of about $1878 \mathrm{Tg} \mathrm{yr}^{-1}$. Additionally, the radical recycling terms (Reactions $\mathrm{R} 1$ and $\mathrm{R} 5$ ) contribute $1987 \mathrm{Tg} \mathrm{yr}^{-1}$, the $\mathrm{H}_{2} \mathrm{O}_{2}$ photodissociation, i.e.,

$\mathrm{H}_{2} \mathrm{O}_{2}+h v \rightarrow 2 \mathrm{OH}$,

produces $303 \mathrm{Tg} \mathrm{yr}^{-1}$, and all other reactions add another $120 \mathrm{Tg} \mathrm{yr}^{-1}$ to the global tropospheric $\mathrm{OH}$ production in the model. Overall, the total tropospheric $\mathrm{OH}$ production amounts to $4288 \mathrm{Tg} \mathrm{yr}^{-1}$, which is in close agreement with the budget estimations by Lelieveld et al. (2016), i.e., $\sim 4270 \mathrm{Tg} \mathrm{yr}^{-1}$. Some difference is, however, expected due to the definition of the troposphere in Lelieveld et al. (2016), who define the tropopause in the tropics using temperature and in the extratropics using potential vorticity gradients. We remind the reader that for the present study the chemical troposphere is defined using a threshold of $150 \mathrm{ppbO}_{3}$. It is striking that the $\mathrm{OH}$ chemical production calculated for the MOGUNTIA model setup is much higher (28\%-35\%) than for previous TM5 model configurations (i.e., $3355 \pm 30$ and $3184 \pm 20 \mathrm{Tg} \mathrm{yr}^{-1}$ ) as presented by van Noije et al. (2014) using a similar $150 \mathrm{ppbO}_{3}$ tropopause. This difference is mainly attributed to the various updates of the model compared to the version used in Noije et al. (2014), such as the emission database and the applied VOC representation (i.e., CMIP5; Lamarque et al., 2010, vs. CMIP6 for this study), the chemistry scheme (i.e., CB4 vs. MOGUNTIA), and the photolysis scheme (i.e., the previous implemented Landgraf et al., 1998, photolysis scheme vs. the modified band approach scheme implemented by Williams et al., 2012).

Focusing on the differences between the MOGUNTIA and mCB05(KPP) mechanism, the MOGUNTIA OH production is very close to mCB05(KPP) on a global scale (Table 5). Note that for mCB05, the comparison of the two solvers indicates that EBI calculates a $\sim 1 \%$ lower chemical destruction of $\mathrm{OH}$ in the troposphere than Rosenbrock. The contributions of the $\mathrm{CO}$ and $\mathrm{CH}_{4}$ oxidation terms to the global tropospheric $\mathrm{OH}$ losses are calculated as $41 \%$ and $15 \%$, respectively, for 
(a)

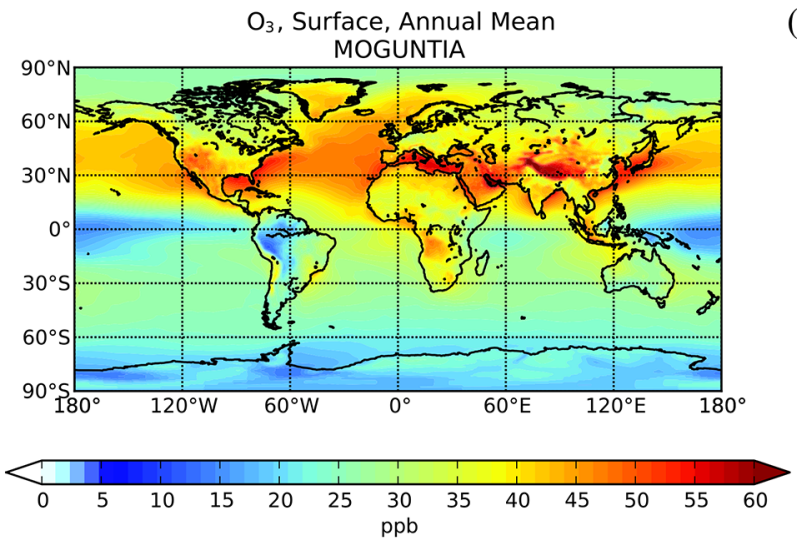

(c)

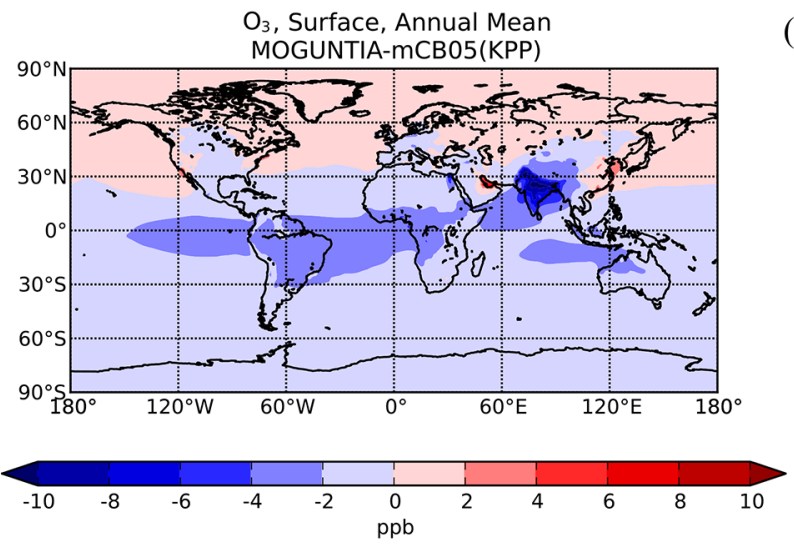

(e)

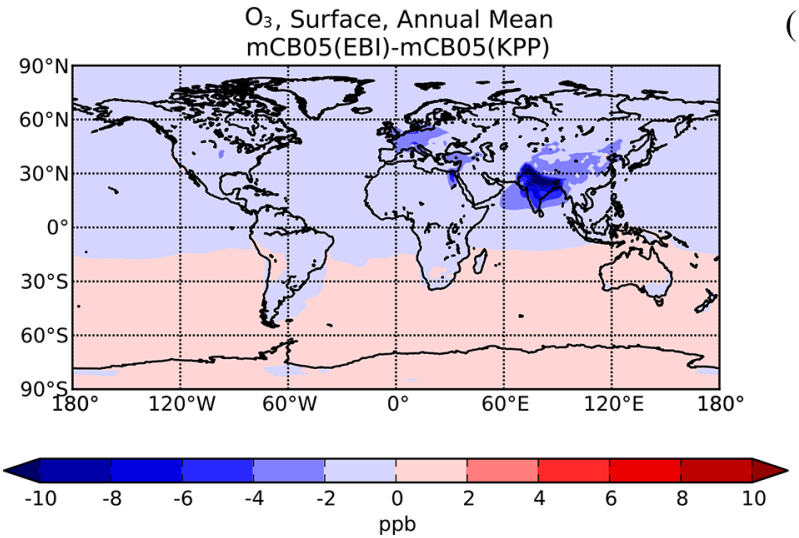

(b)

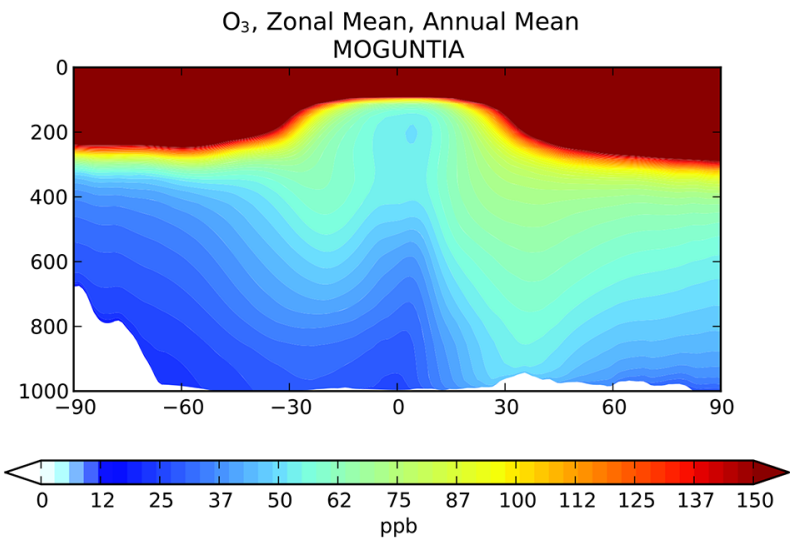

(d)

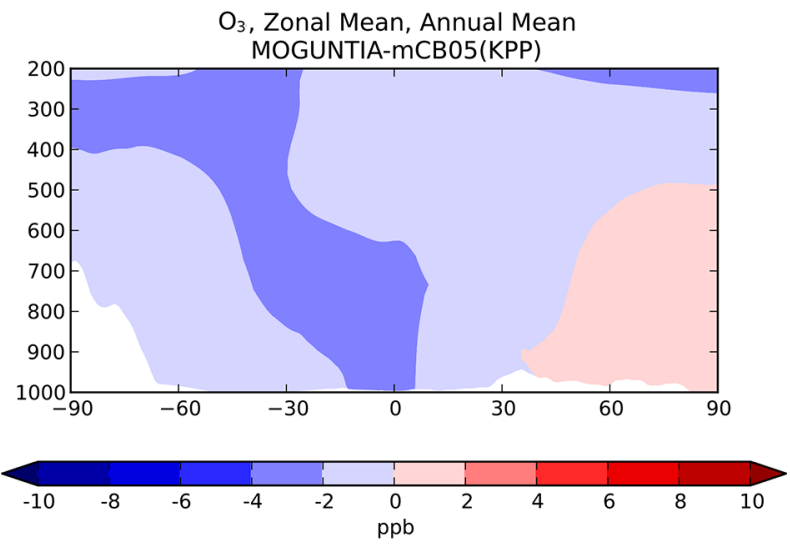

(f)

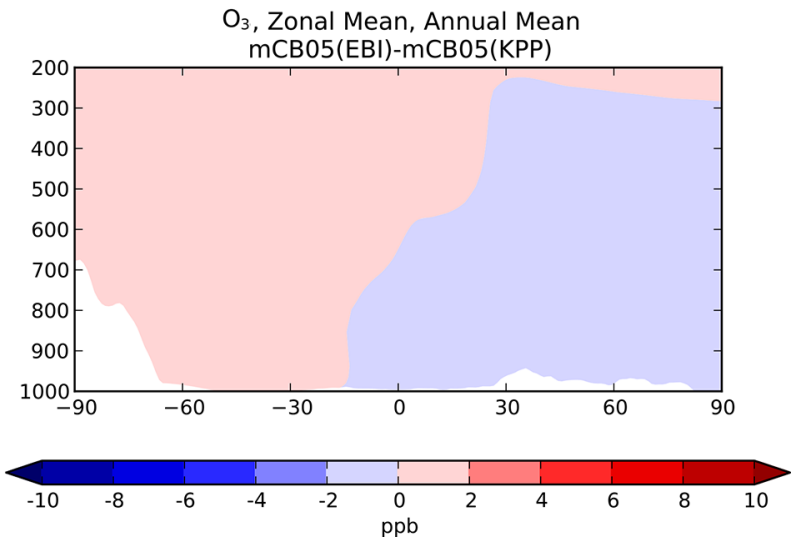

Figure 1. Simulated annual mean surface (a, c, e) and zonal mean (b, d, f) $\mathrm{O}_{3}$ mixing ratios (ppb) for the MOGUNTIA chemistry scheme for the year 2006 (a, b) and the respective differences compared to mCB05(KPP) (c, d); the surface and zonal mean absolute differences between $\mathrm{mCB} 05(\mathrm{KPP})$ and $\mathrm{mCB} 05(\mathrm{EBI})$ are also presented $(\mathbf{e}, \mathbf{f})$.

the MOGUNTIA scheme. This is slightly higher (by $\sim 6 \%$ and $\sim 3 \%$, respectively) compared to $\mathrm{mCB} 05(\mathrm{KPP})$.

Focusing further on the MOGUNTIA scheme, the calculated tropospheric $\mathrm{CH}_{4}$ chemical lifetime is $\sim 8.0$ years, as obtained by dividing the $\mathrm{CH}_{4}$ global atmospheric mean burden $(\sim 4871 \mathrm{Tg})$ by the loss due to oxidation by $\mathrm{OH}$ radicals in the troposphere $\left(\sim 607 \mathrm{Tg} \mathrm{yr}^{-1}\right)$. Accounting, however, for additional $\mathrm{CH}_{4}$ sinks due to oxidation in soils and the stratosphere with assumed lifetimes of 160 and 120 years
(Ehhalt et al., 2001), respectively, an atmospheric lifetime of about 7.18 years is derived, which is roughly $15 \%$ shorter than the ensemble model mean atmospheric lifetime reported by Stevenson et al. (2006) of $8.45 \pm 0.38$ years. The multimodel chemistry-climate simulations performed during the Atmospheric Chemistry and Climate Model Intercomparison Project (ACCMIP) (Naik et al., 2013; Voulgarakis et al., 2013) revealed vast diversities among models, with a wide range of $\mathrm{CH}_{4}$ chemical lifetime values (i.e., 7-14 years) 
Table 5. Tropospheric chemical budget of $\mathrm{OH}$ for the year $2006\left(\mathrm{Tg}(\mathrm{OH}) \mathrm{yr}^{-1}\right)$ using the $150 \mathrm{ppb}_{3}$ mixing ratio to define the tropopause level. In parentheses, the relative differences using the $100 \mathrm{ppb} \mathrm{O}_{3}$ mixing ratios are also presented, calculated with reference to the $150 \mathrm{ppb}$ $\mathrm{O}_{3}$ tropopause level definition.

\begin{tabular}{|c|c|c|c|c|c|c|c|c|c|c|c|c|c|}
\hline \multirow{2}{*}{$\begin{array}{l}\text { Production terms } \\
\mathrm{O}\left({ }^{1} \mathrm{D}\right)+\mathrm{H}_{2} \mathrm{O}\end{array}$} & \multicolumn{2}{|c|}{ mCB05 (EBI) } & \multicolumn{2}{|c|}{ mCB05 (KPP) } & \multicolumn{2}{|c|}{ MOGUNTIA } & \multirow{2}{*}{$\begin{array}{l}\text { Loss terms } \\
\mathrm{OH}+\mathrm{CO}\end{array}$} & \multicolumn{2}{|c|}{ mCB05 (EBI) } & \multicolumn{2}{|c|}{ mCB05 (KPP) } & \multicolumn{2}{|c|}{ MOGUNTIA } \\
\hline & 1960 & $(0 \%)$ & 1953 & $(0 \%)$ & 1878 & $(0 \%)$ & & 1665 & $(-2 \%)$ & 1671 & $(-2 \%)$ & 1775 & $(-2 \%)$ \\
\hline $\mathrm{NO}+\mathrm{HO}_{2}$ & 1268 & $(-4 \%)$ & 1312 & $(-4 \%)$ & 1426 & $(-4 \%)$ & $\mathrm{OH}+\mathrm{CH}_{4}$ & 613 & $(0 \%)$ & 626 & $(0 \%)$ & 644 & $(-1 \%)$ \\
\hline $\mathrm{O}_{3}+\mathrm{HO}_{2}$ & 560 & $(-1 \%)$ & 566 & $(-1 \%)$ & 561 & $(-1 \%)$ & $\mathrm{OH}+\mathrm{O}_{3}$ & 254 & $(-2 \%)$ & 260 & $(-2 \%)$ & 262 & $(-3 \%)$ \\
\hline $\mathrm{H}_{2} \mathrm{O}_{2}+h v$ & 262 & $(-1 \%)$ & 265 & $(-1 \%)$ & 303 & $(-1 \%)$ & $\mathrm{OH}+\mathrm{ISOP}$ & 114 & $(-1 \%)$ & 115 & $(-1 \%)$ & 120 & $(0 \%)$ \\
\hline Other & 203 & $(-2 \%)$ & 201 & $(-2 \%)$ & 120 & $(-1 \%)$ & Other & 1606 & $(-1 \%)$ & 1626 & $(-1 \%)$ & 1487 & $(-1 \%)$ \\
\hline
\end{tabular}

and a mean value of $9.7 \pm 1.5$ years (i.e., $5 \%-10 \%$ higher than observation-derived estimates). Lelieveld et al. (2016) derived a $\mathrm{CH}_{4}$ chemical lifetime of 8.5 years for the year 2010, and Schultz et al. (2018) estimated a tropospheric $\mathrm{CH}_{4}$ chemical lifetime of about 9.9 years also using an $\mathrm{O}_{3}$ threshold of $150 \mathrm{ppb}$ to define the tropopause. Finally, Lamarque et al. (2012) reported a chemical lifetime of $\sim 8.7$ years by taking a tropopause level at $100 \mathrm{ppb} \mathrm{O}_{3}$.

\subsection{Carbon monoxide (CO)}

Table 6 presents the chemical $\mathrm{CO}$ budget calculated by TM5-MP for the three chemical configurations. The different model configurations show that approximately $62 \pm 1 \%$ of the $\mathrm{CO}$ global production in the troposphere is due to the oxidation of $\mathrm{CH}_{4}$ and NMVOCs, with the remaining due to direct emissions. Overall, the global CO budget is significantly affected by the interactions between $\mathrm{OH}$ and $\mathrm{CO}$. Thus, changes in $\mathrm{OH}$ tropospheric chemical production (i.e., $\sim-0.2 \%$ from mCB05(KPP) to MOGUNTIA) modulate the tropospheric secondary formation of $\mathrm{CO}$ from the oxidation of $\mathrm{CH}_{4}$ and NMVOCs ( $\sim-10 \%$ change) as well as the $\mathrm{CO}$ chemical loss $(\sim-3 \%$ change $)$ in the model. The global chemical production (i.e., the sum of chemical production terms in the troposphere and stratosphere; Table 6) of CO for both the MOGUNTIA and mCB05(KPP) chemical configurations, i.e., 2018 and $1844 \mathrm{Tg} \mathrm{yr}^{-1}$, respectively, is, however, higher than the multi-model mean estimate $\left(1505 \pm 236 \mathrm{Tg} \mathrm{yr}^{-1}\right)$ reported by Shindell et al. (2006), which can be partially attributed to the different year of NMVOC emissions used (i.e., 2000 vs. 2006 for this work).

The dominant chemical reaction responsible for the increase in tropospheric $\mathrm{CO}$ chemical production for MOGUNTIA compared to the mCB05(KPP) chemical configuration is the $\mathrm{HCHO}$ oxidation by $\mathrm{OH}$ radicals (i.e., $\sim 15 \%$ increase compared to mCB05(KPP)). Indeed, although the lumped nature of the $\mathrm{mCB} 05(\mathrm{KPP})$ mechanism leads to a higher tropospheric $\mathrm{HCHO}$ chemical production $\left(\sim 1896 \mathrm{Tg} \mathrm{yr}^{-1}\right)$ compared to the MOGUNTIA configuration $\left(\sim 1843 \mathrm{Tg} \mathrm{yr}^{-1}\right)$, the HCHO tropospheric chemical destruction is calculated roughly $2 \%$ higher for the MOGUNTIA scheme. HCHO is mainly formed via the oxidation of $\mathrm{CH}_{4}$, isoprene, and other NMVOCs in the model. However, for both mCB05 configurations, the $\mathrm{HCHO}$ production via $\mathrm{CH}_{3} \mathrm{O}_{2} \mathrm{H}$ photolysis is calculated to be $\sim 1.65$ times higher compared to MOGUNTIA. The latter scheme seems to recycle the methyl-peroxy radical $\left(\mathrm{CH}_{3} \mathrm{O}_{2}\right)$ more efficiently via $\mathrm{CH}_{3} \mathrm{O}_{2}$ gas-phase reactions with organic peroxy radicals $\left(\mathrm{RO}_{2}\right)$ produced by higher-order NMVOC oxidation. In contrast, other higher aldehydes that represent the second-most important producer of $\mathrm{CO}$ contribute more significantly in MOGUNTIA than in mCB05. This could be due to the more detailed representation of the higher aldehydes in the MOGUNTIA mechanism (e.g., considering the production and destruction reaction of GLY, GLYAL, and $\mathrm{C}_{2} \mathrm{H}_{5} \mathrm{CHO}$ ) compared to the single lumped species (i.e., the ALD2) that represents all higher aldehydes in mCB05.

The global annual mean burden of CO for the MOGUNTIA chemical scheme is $361 \mathrm{Tg}$, almost the same as in the mCB05(KPP) configuration but $\sim 2 \%$ lower compared to mCB05(EBI). Higher CO losses by OH oxidation and deposition in MOGUNTIA lead to a CO atmospheric lifetime of $\sim 44$ d, i.e., about $6 \%$ shorter compared to the mCB05(KPP) chemical mechanism. Note that the reduction in the atmospheric lifetime of $\mathrm{CO}$ is in line with the reduction in the atmospheric lifetime of $\mathrm{CH}_{4}(\sim 3 \%)$, reflecting an overall increase in tropospheric $\mathrm{OH}$ mixing ratios for the MOGUNTIA configuration compared to mCB05(KPP); i.e., higher $\mathrm{OH}$ levels in the atmosphere lead to proportionally larger $\mathrm{CO}$ and $\mathrm{CH}_{4}$ sinks.

Focusing further on the impact of the solver alone, we calculate roughly a $3 \%$ reduction in the $\mathrm{CO}$ atmospheric burden when the EBI solver is applied to the mCB05 mechanism in the model. This is directly connected to the $\sim 1 \%$ increase in $\mathrm{OH}$ mixing ratios that is calculated when the Rosenbrock solver is used in the model. Furthermore, the CO tropospheric production is increased by $\sim 0.5 \%$ in $\mathrm{mCB} 05$ (KPP) compared to mCB05(EBI). Overall, the presented differences between the EBI and Rosenbrock solvers confirm that the choice of solver may impact the simulated mixing ratios, owing mainly to the use of a constant versus a variable time step in the chemistry integration (see, e.g., Sandu et al., 1997).

Zonal mean $\mathrm{CO}$ mixing ratios at the surface for the year 2006 using the MOGUNTIA scheme are presented in Fig. 2a and b. Compared to mCB05(KPP), the results from MO- 
Table 6. Global budgets and burden $(\mathrm{Tg}(\mathrm{CO}))$ of $\mathrm{CO}$ for the year $2006\left(\mathrm{Tg}(\mathrm{CO}) \mathrm{yr}^{-1}\right)$ using the $150 \mathrm{ppb} \mathrm{O}_{3}$ mixing ratio to define the tropopause level. In parentheses, the relative differences using the $100 \mathrm{ppb} \mathrm{O}_{3}$ mixing ratios are also presented, calculated with reference to the $150 \mathrm{ppb} \mathrm{O}_{3}$ tropopause level definition.

\begin{tabular}{|c|c|c|c|c|c|c|c|c|c|c|c|c|c|}
\hline \multirow{2}{*}{$\begin{array}{l}\text { Production terms } \\
\text { Emissions }\end{array}$} & \multicolumn{2}{|c|}{ mCB05 (EBI) } & \multicolumn{2}{|c|}{ mCB05 (KPP) } & \multicolumn{2}{|c|}{ MOGUNTIA } & \multirow{2}{*}{$\begin{array}{l}\text { Loss terms } \\
\text { Deposition }\end{array}$} & \multicolumn{2}{|c|}{ mCB05 (EBI) } & \multicolumn{2}{|c|}{ mCB05 (KPP) } & \multicolumn{2}{|c|}{ MOGUNTIA } \\
\hline & 1097 & $(0 \%)$ & 1097 & $(0 \%)$ & 1097 & $(0 \%)$ & & 98 & $(0 \%)$ & 97 & $(0 \%)$ & 99 & $(0 \%)$ \\
\hline Trop. chem. production & 1809 & $(-1 \%)$ & 1818 & $(-1 \%)$ & 1992 & $(-1 \%)$ & Trop. chem. loss & 2840 & $(-6 \%)$ & 2849 & $(-6 \%)$ & 2924 & $(-2 \%)$ \\
\hline Strat. chem. production & 26 & $(69 \%)$ & 26 & $(73 \%)$ & 26 & $(65 \%)$ & Strat. chem. loss & 87 & $(68 \%)$ & 89 & $(69 \%)$ & 90 & $(68 \%)$ \\
\hline Atmos. burden & 370 & $(0 \%)$ & 360 & $(0 \%)$ & 361 & $(0 \%)$ & Lifetime (days) & 47.5 & $(2 \%)$ & 46.2 & $(2 \%)$ & 43.6 & $(3 \%)$ \\
\hline
\end{tabular}

GUNTIA show slightly higher surface CO mixing ratios (up to $\sim 2 \mathrm{ppb}$ ) over highly populated regions, such as India. This regional increase is due to the differences in surface $\mathrm{OH}$ mixing ratios, owing mainly to the differences in $\mathrm{NO}_{x}$ chemistry between the two simulations (see also Sect. 5.2). In contrast, in South America negative differences of $\sim 5-15 \mathrm{ppb}$ are calculated at the surface (Fig. 2c). The effective $\mathrm{HO}_{x}$ regeneration together with the detailed VOC representation and oxidation pathways considered in MOGUNTIA result in an increase in the surface $\mathrm{OH}$ mixing ratios in locations with high biogenic VOC emissions. This subsequently leads to a regional decrease in the tropospheric $\mathrm{CO}$ mixing ratios compared to the mCB05(KPP) configuration. Similar results are found for the zonal mean $\mathrm{CO}$ distribution. Free tropospheric $\mathrm{CO}$ mixing ratios in the tropics are also affected due to effective tropical convection. Finally, the use of different solvers for the mCB05 mechanism does not lead to any notable differences in the annual mean $\mathrm{CO}$ mixing ratios (Fig. 2e, f).

\section{Model evaluation}

Model simulations are evaluated with a series of surface, flask, aircraft, and sonde measurements, as well as with satellite retrievals and climatological data. The simulated $\mathrm{NO}_{2}$ tropospheric columns are compared with satellite retrievals from the European Quality Assurance for Essential Climate Variables (QA4ECV) project (Boersma et al., 2017), provided by the Ozone Monitoring Instrument (OMI) and the SCanning Imaging Absorption SpectroMeter for Atmospheric CHartographY (SCIAMACHY). The simulated OH mixing ratios are evaluated against calculations of global mean tropospheric values from other modeling studies and against climatological data compiled by Spivakovsky et al. (2000). Modeled $\mathrm{O}_{3}$ mixing ratios are evaluated against surface observations and ozonesonde data for the year 2006, as compiled by the World Ozone and Ultraviolet Radiation Data Centre (WOUDC; http://www.woudc.org; last access: 20 August 2019); surface observations from the European Monitoring Evaluation Program network (EMEP; http: //www.emep.int; last access: 20 August 2019) have been also used. For the $\mathrm{CO}$ model evaluation, flask observations for the year 2006 are used, as compiled by the National Oceanic and Atmospheric Administration Earth System Research Laboratory, Global Monitoring Division (NOAA; https://www. esrl.noaa.gov/gmd; last access: 20 August 2019). $\mathrm{O}_{3}$ and $\mathrm{CO}$ mixing ratios in the upper troposphere-lower stratosphere (UTLS) are compared to in situ measurements from the MOZAIC (Measurement of Ozone and Water Vapour by Airbus In-Service Aircraft) data record (Thouret et al., 1998). The modeled CO total columns are compared with satellite retrievals from the Measurement of Pollution in the Troposphere (MOPITT) instrument version MOP02J_V008 (Deeter et al., 2013, 2019; Ziskin, 2019), i.e., the combined thermal-near-infrared data product. Finally, light VOCs (i.e., $\mathrm{C}_{2} \mathrm{H}_{4}, \mathrm{C}_{2} \mathrm{H}_{6}, \mathrm{C}_{3} \mathrm{H}_{6}, \mathrm{C}_{3} \mathrm{H}_{8}$ ) as simulated for the year 2006 are evaluated against flask measurements from the NOAA database and against climatological data from aircraft campaigns, as produced by Emmons et al. (2000). Overall, to quantify and discuss the model performance, commonly used statistical parameters are calculated, such as the correlation coefficient $(R)$, which reflects the strength of the linear relationship between model results and observations (the ability of the model to simulate the observed variability), the absolute bias (BIAS), the normalized mean bias (NMB), and the root mean square error (RMSE) as a measure of the mean deviation of the model from the measurement due to random and systematic errors. All equations used for the statistical analysis of model results are provided in the Supplement (Eqs. S1-S5).

\subsection{Nitrogen dioxide $\left(\mathrm{NO}_{2}\right)$}

$\mathrm{NO}_{x}$ is a rate-limiting precursor of $\mathrm{O}_{3}$ formation and thus an essential species for other tropospheric oxidants, such as $\mathrm{OH} . \mathrm{NO}_{x}$ is emitted by both natural (lightning, soils, and fires) and anthropogenic combustion sources, with lightning mainly impacting $\mathrm{NO}_{x}$ mixing ratios at the top of convective updrafts and anthropogenic fuel emissions being the principal source of $\mathrm{NO}$ at the surface. Tropospheric $\mathrm{NO}_{2}$ vertical column densities retrieved from OMI (Boersma et al., 2017) are compared against the MOGUNTIA and mCB05(KPP) simulations (Fig. 3). Note that since the differences between $\mathrm{mCB} 05(\mathrm{EBI})$ and $\mathrm{mCB} 05(\mathrm{KPP})$ are small for tropospheric $\mathrm{NO}_{2}$ columns, mCB05(EBI) is not shown. $\mathrm{NO}_{2}$ column densities are retrieved using a consistent set of retrieval parameters and validated against ground-based MAX-DOAS measurements (Boersma et al., 2018). To consider the vertical sensitivity of the satellite measurements to $\mathrm{NO}_{2}$ molecules 
(a)
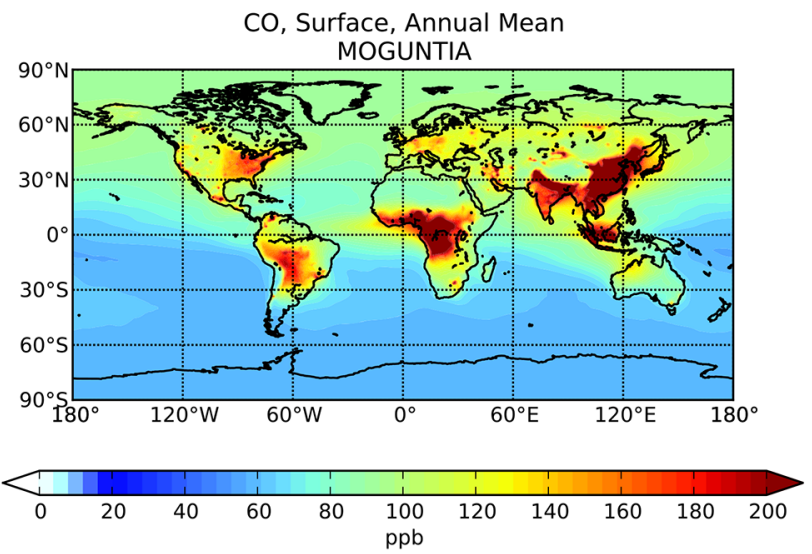

(c)
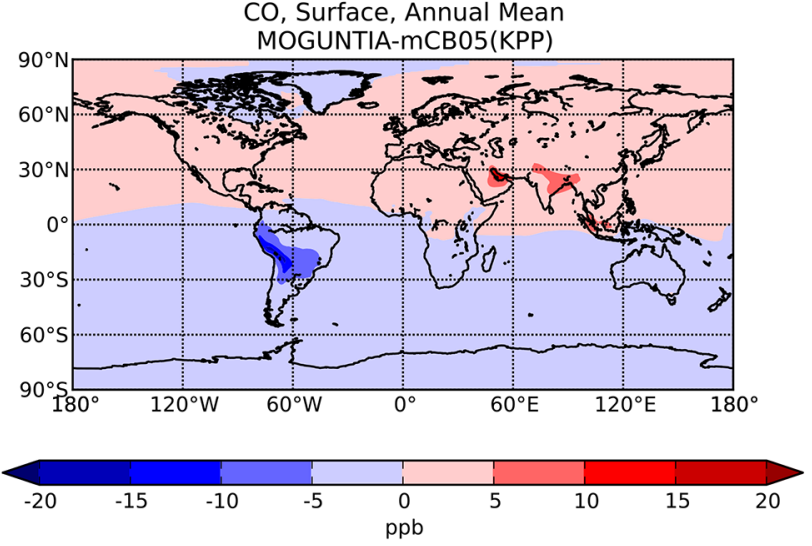

(e)
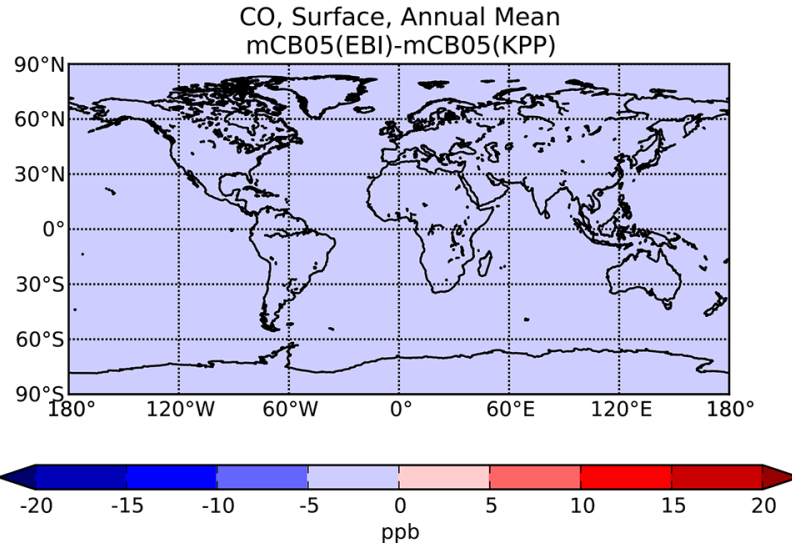

(b)
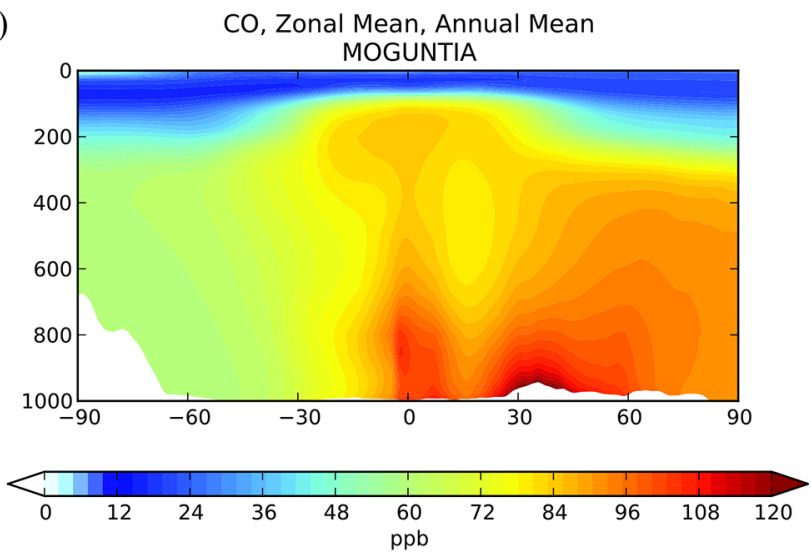

(d)

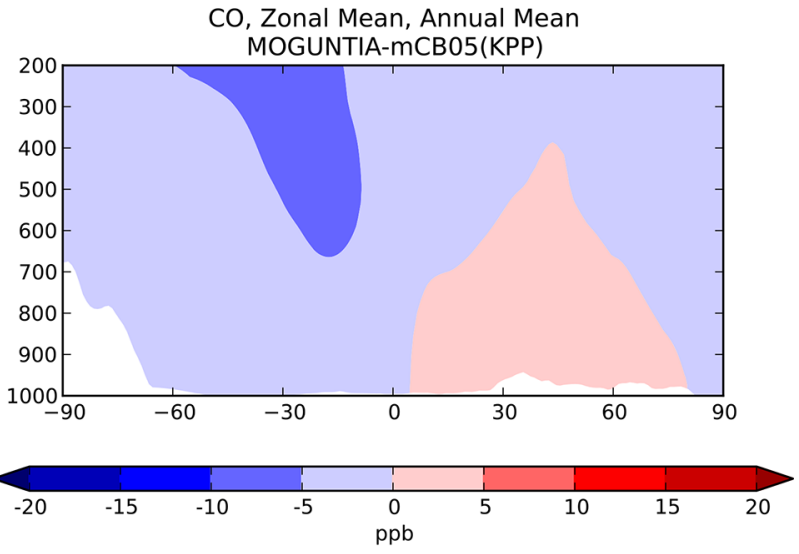

(f)

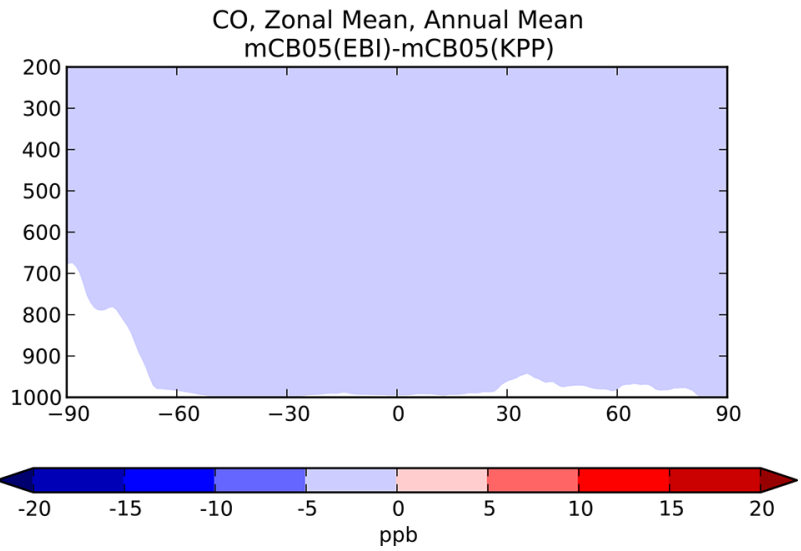

Figure 2. Simulated annual mean surface $(\mathbf{a}, \mathbf{c}, \mathbf{e})$ and zonal mean $(\mathbf{b}, \mathbf{d}, \mathbf{f}) \mathrm{CO}$ mixing ratios (ppb) for the MOGUNTIA chemistry scheme for the year $2006(\mathbf{a}, \mathbf{b})$ and the respective differences compared to mCB05(KPP) (c, d); the surface and zonal mean absolute differences between $\mathrm{mCB} 05(\mathrm{KPP})$ and $\mathrm{mCB} 05(\mathrm{EBI})$ are also presented (e, f).

at different altitudes, the tropospheric column averaging kernels provided in the QA4ECV data product are applied separately to both sets of modeled $\mathrm{NO}_{2}$ vertical profiles, extracted from the hourly 3-D model output by linear and nearestneighbor interpolation in space and time. The resulting $\mathrm{NO}_{2}$ tropospheric column density is what would have been retrieved by the satellite if the actual vertical profile of $\mathrm{NO}_{2}$ mixing ratios were identical to the modeled profile. The tro- pospheric $\mathrm{NO}_{2}$ columns retrieved from the satellite are averaged per model grid cell and day, resulting in a comparison dataset consisting of one $\mathrm{NO}_{2}$ vertical column density per model grid cell and day.

For the MOGUNTIA configuration, the model shows a mean overestimation of $1.78 \times 10^{14}(R=0.71)$ and $1.96 \times$ $10^{14}$ molec. $\mathrm{cm}^{-2}(R=0.95)$ against OMI measurements for daily and annual values, respectively, performing slightly 
MOGUNTIA

(a)

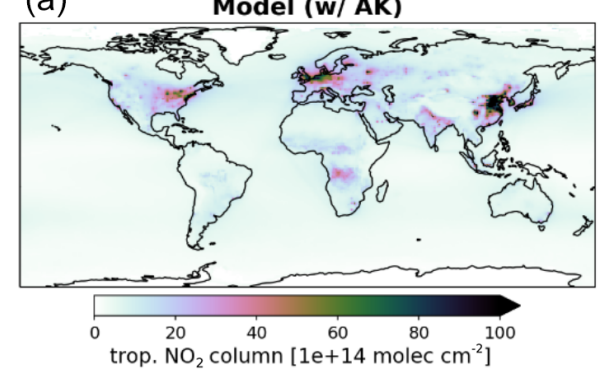

(c)

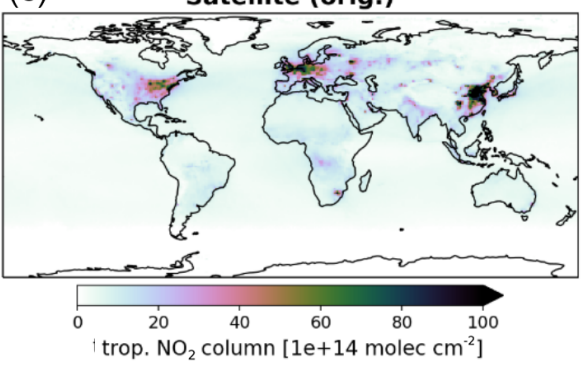

(e)

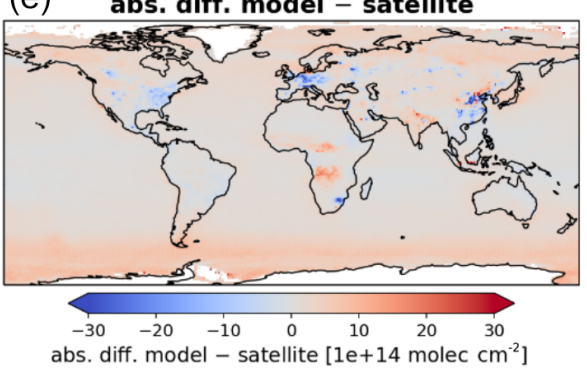

(g)

rel. diff. model - satellite

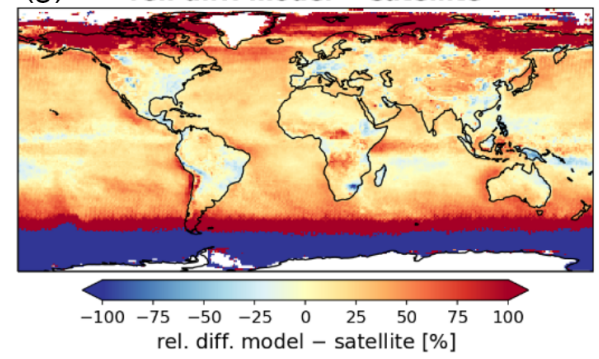

$\mathrm{mCB05}(\mathrm{KPP})$

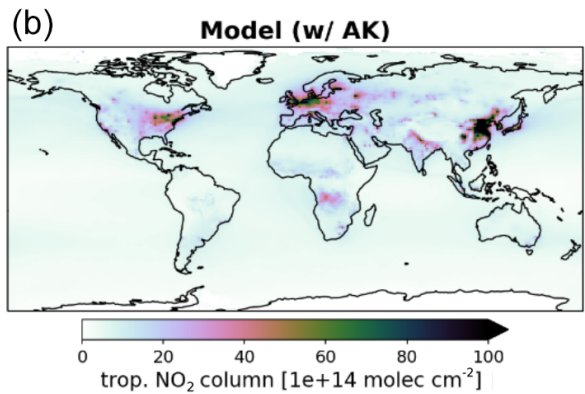

(d)

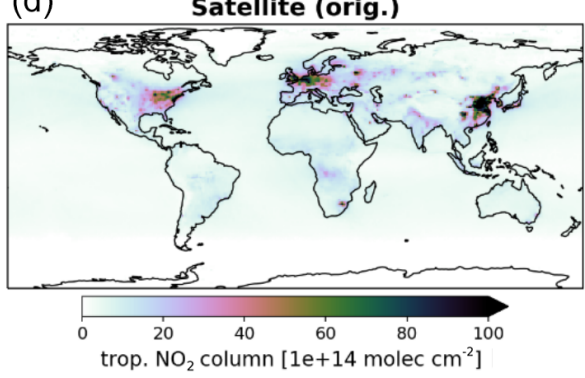

(f) abs. diff. model - satellite

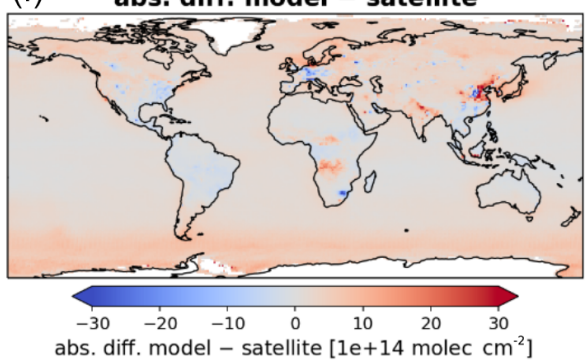

(h) rel. diff. model - satellite

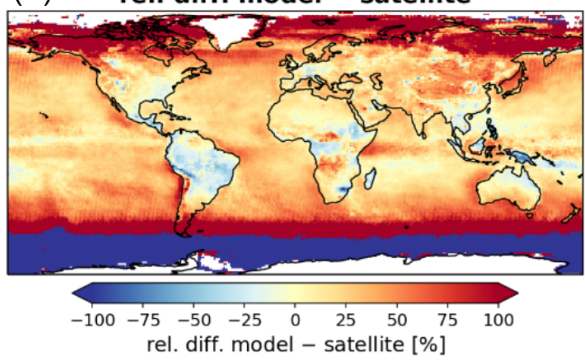

Figure 3. Annual mean comparison of tropospheric $\mathrm{NO}_{2}$ vertical columns $\left(\right.$ molec. $\mathrm{cm}^{-2}$ ) for the two chemistry schemes MOGUNTIA and mCB05(KPP) (a, b) against the Ozone Monitoring Instrument (OMI) satellite data (c, d) using the respective averaging kernel information for 2006. The absolute $(\mathbf{e}, \mathbf{f})$ and relative $(\mathbf{g}, \mathbf{h})$ differences are also presented.

better than the correlation of the mCB05(KPP) configuration ( $R=0.71$ and $R=0.94$ for daily and annual values). An overview of the statistical comparison of the three model simulations against OMI measurements is given in Fig. S1a. Some discrepancies, especially in the Northern Hemisphere $(\mathrm{NH})$, may be attributed to the absence of a significant seasonal cycle in monthly anthropogenic emissions. Over the biomass burning source regions in Africa, the model overestimates the satellite retrievals. When the model is compared against $\mathrm{NO}_{2}$ tropospheric columns from the SCIAMACHY instrument using the QA4ECV retrieval (not shown), the MOGUNTIA configuration shows a similar improvement over mCB05(KPP), as with the OMI data. 
Williams et al. (2017) showed that the TM5-MP model significantly underestimates the $\mathrm{NO}$ and $\mathrm{NO}_{2}$ mixing ratios, both at the surface and in vertical profiles. The model satisfactorily reproduces the $\mathrm{NO}_{2}$ mixing ratios in the boundary layer but overestimates mixing ratios at higher altitudes and in pristine environments. The MOGUNTIA scheme shows generally better agreement with satellite retrievals compared to the mCB05(KPP) configuration, as expressed by a higher correlation coefficient and a generally lower bias (Fig. S1a). The differences between the two chemistry schemes can be mainly attributed to the representation of organic $\mathrm{NO}_{x}$ reservoir species (i.e., the organic nitrates; ORGNTRs) in the two mechanisms (Fig. S2). Overall, since deep convection may efficiently transport ORGNTRs to the upper troposphere, the more explicit representation of VOC chemistry in the MOGUNTIA chemistry scheme alters the distribution of ORGNTRs compared to the more lumped chemistry of mCB05. Although production of ORGNTRs is about $10 \%$ larger in the MOGUNTIA scheme, the ORGNTR burden is dominated by the loss term (Table S4). Due to the more detailed ORGNTR representation in the MOGUNTIA scheme, the destruction becomes significantly more efficient compared to the mCB05 configuration. As a result, the global ORGNTR burden calculated using the MOGUNTIA scheme in the model is about $60 \%$ smaller.

Several modeling studies have compared simulated $\mathrm{NO}_{2}$ columns with in situ and satellite observations (e.g., Travis et al., 2016; Williams et al., 2017). These studies demonstrated an overestimate of the observed $\mathrm{NO} / \mathrm{NO}_{2}$ ratios compared to observations at higher altitudes, possibly due to a respective underestimate of peroxy radicals in the upper troposphere that contributes to the $\mathrm{NO}$ to $\mathrm{NO}_{2}$ conversion. A deviation in the $\mathrm{NO} / \mathrm{NO}_{2}$ ratio has also been reported for the GEOS-Chem model (Silvern et al., 2018; Travis et al., 2016). This model significantly underestimated the observed upper tropospheric $\mathrm{NO}_{2}$ observations from the SEAC ${ }^{4} \mathrm{RS}$ aircraft campaign over the southeast United States. Silvern et al. (2018) calculated the reaction with ozone to account for roughly $75 \%$ of the $\mathrm{NO}$ to $\mathrm{NO}_{2}$ conversion in the upper troposphere; thus, this deviation from the photochemical equilibrium could be due to an error in kinetic data. Overall, the authors indicated that reducing the $\mathrm{NO}_{2}$ photolysis by $20 \%$ and increasing the low-temperature $\mathrm{NO}+\mathrm{O}_{3}$ reaction rate constant by $40 \%$ improves the model simulation of the $\mathrm{NO} / \mathrm{NO}_{2}$ ratio in the upper tropospheric data significantly compared to the aircraft data. Another source of uncertainty could be the strength of the direct soil emissions that, according to Miyazaki et al. (2017), are lower in our model (i.e., $\sim 5 \mathrm{Tg} \mathrm{Nyr}^{-1}$; Yienger and Levy, 1995) compared to the emissions of $7.9 \mathrm{Tg} \mathrm{N} \mathrm{yr}^{-1}$ derived using a multiconstituent satellite data assimilation.

\subsection{Hydroxyl radical $(\mathrm{OH})$}

Figure $4 \mathrm{a}$ and $\mathrm{b}$ illustrate the zonal mean tropospheric distributions of $\mathrm{OH}$ for two seasons (i.e., boreal winter and boreal summer) for 2006, as simulated with the MOGUNTIA chemistry scheme. The highest atmospheric mixing ratios of $\mathrm{OH}$ in the model are calculated in the tropics from close to the surface up to roughly the tropopause as a result of intense solar radiation and high humidity in the region, with the main $\mathrm{OH}$ maximum being roughly below $400 \mathrm{hPa}$ (and a secondary maximum at $\sim 300 \mathrm{hPa}$ ). The differences in $\mathrm{OH}$ zonal mean mixing ratios compared to the mCB05(KPP) configuration are presented in Fig. $4 \mathrm{c}$ and d. During the boreal winter, the mCB05(KPP) configuration results on average in lower $\mathrm{OH}$ mixing ratios in the northern subtropical lower troposphere $(\sim 3 \%-6 \%)$ than the MOGUNTIA simulation (Fig. $4 \mathrm{c}$ ), with the largest differences ( 20\%-30\%) around $20-40^{\circ} \mathrm{N}$. In the subtropical Southern Hemisphere (SH) during boreal summer, $\mathrm{OH}$ mixing ratios are on average lower $(\sim 2 \%-3 \%)$ in the MOGUNTIA configuration than in $\mathrm{mCB} 05$ (KPP) (Fig. 4d) almost everywhere, except for a small increase (up to $10 \%$ ) at around $30^{\circ} \mathrm{S}$. These small differences in $\mathrm{OH}$ mixing ratios are mainly related to the $\mathrm{HO}_{x}$ regeneration and differences in $\mathrm{NO}_{x}$ and ORGNTR species that influence the distribution of $\mathrm{OH}$ in the troposphere. The more detailed representation of ORGNTRs in the MOGUNTIA chemistry scheme results in more efficient $\mathrm{NO}_{x}$ release upon ORGNTR destruction (Table S4), leading overall to $\mathrm{O}_{3}$ formation in remote locations and thus to the stimulation of $\mathrm{HO}_{x}$ recycling at higher altitudes. Note that globally the $\mathrm{NO}+\mathrm{HO}_{2}$ reaction is roughly $9 \%$ higher in the MOGUNTIA configuration on an annual basis compared to mCB05(KPP) (see Table 5).

Focusing on global means, a global mean tropospheric $\mathrm{OH}$ concentration of $10.1 \times 10^{5}$ molec. $\mathrm{cm}^{-3}$ is obtained from the MOGUNTIA chemistry configuration for the year 2006, which is roughly $4 \%$ higher than in the mCB05(KPP) configuration but closer to the low end of the multi-model mean of $11 \pm 1.6 \times 10^{5}$ molec. $\mathrm{cm}^{-3}$ as derived by Naik et al. (2013) for the year 2000 and the mean tropospheric mixing ratios of $11.3 \times 10^{5}$ molec. $\mathrm{cm}^{-3}$ as calculated by Lelieveld et al. (2016) for the year 2013. In the tropical troposphere $\left(30^{\circ} \mathrm{S}-30^{\circ} \mathrm{N}\right)$, the mean $\mathrm{OH}$ level in the MOGUNTIA configuration of $16.74 \times 10^{5}$ molec. $\mathrm{cm}^{-3}$ is $\sim 6 \%$ higher than in mCB05(KPP). In all model configurations, higher $\mathrm{OH}$ mixing ratios are calculated in the $\mathrm{NH}$ compared to the $\mathrm{SH}$, which is directly related to the asymmetry in the hemispheric $\mathrm{O}_{3}$ and $\mathrm{NO}_{x}$ burdens. Figure $4 \mathrm{e}$ and $\mathrm{f}$ show the climatological mean $\mathrm{OH}$ mixing ratios from the surface up to $\sim 200 \mathrm{hPa}$ from Spivakovsky et al. (2000), reduced by $8 \%$ based on the observed decay of methyl-chloroform mixing ratios (see Huijnen et al., 2010; van Noije et al., 2014). The mean tropospheric $\mathrm{OH}$ concentration for the MOGUNTIA configuration is calculated to be roughly $25 \%$ and $30 \%$ higher compared to the optimized climatology from Spivakovsky et al. (2000) for 
(a)

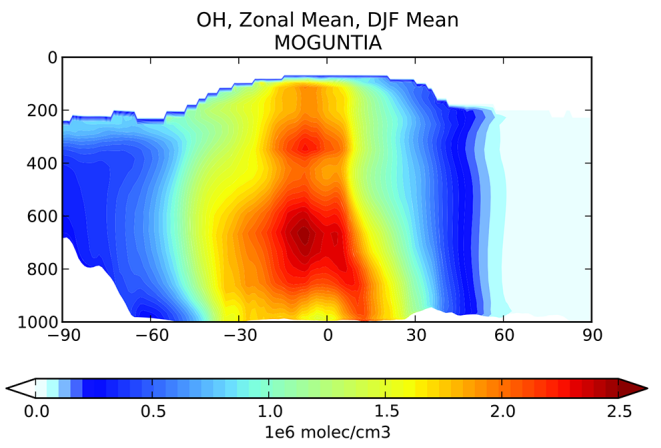

(c)

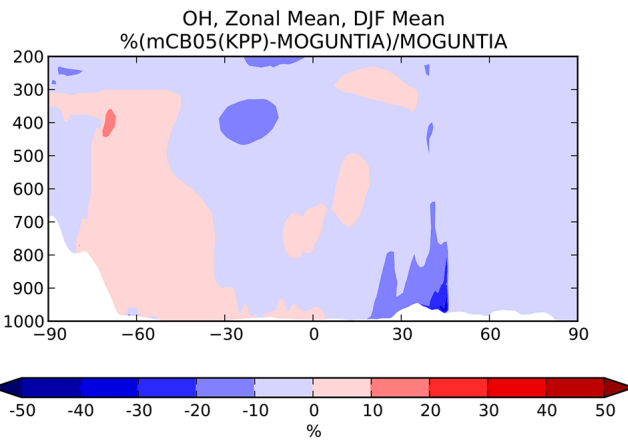

(e)

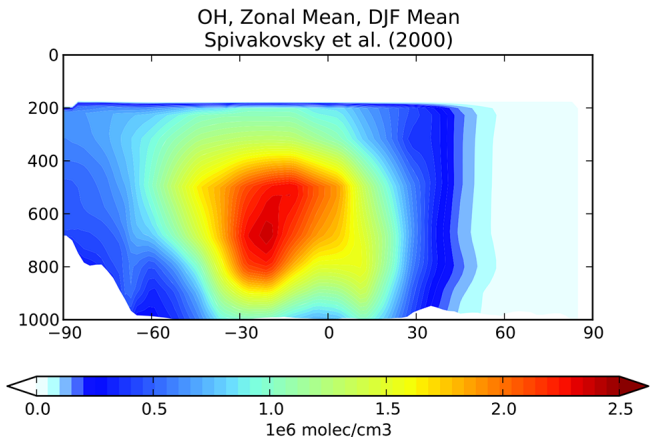

(b)

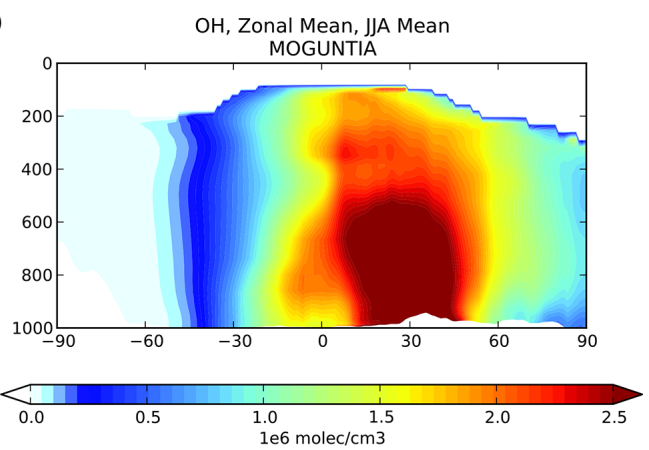

(d)

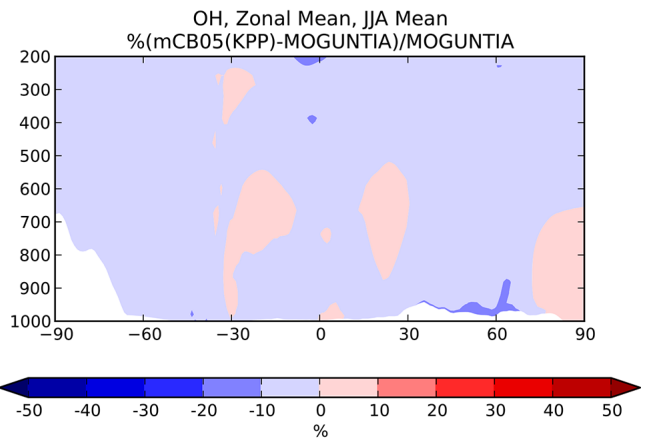

(f)

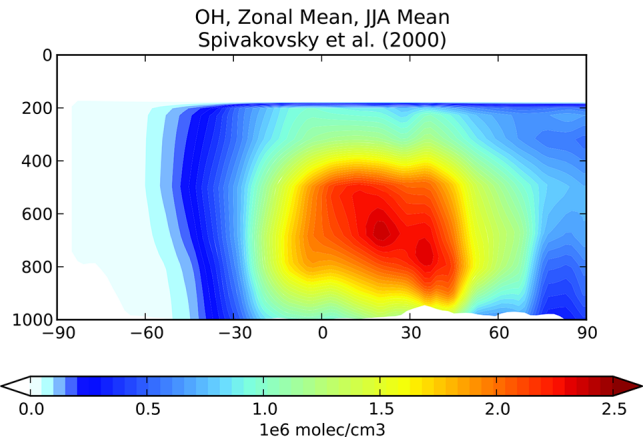

Figure 4. Zonal mean OH mixing ratios for December-January-February (DJF; a, c, e) and June-July-August (JJA; b, d, f) 2006, as simulated by the TM5-MP model with the MOGUNTIA chemistry scheme (a, b). The differences (\%) between the mCB05(KPP) and the MOGUNTIA chemical configuration (c, d) and the optimized climatological average from Spivakovsky et al. (2000) up to 200 hPa (e, f).

boreal winter and summer, respectively. Moreover, a $\sim 28 \%$ higher $\mathrm{NH} / \mathrm{SH}$ ratio of annual mean hemispheric $\mathrm{OH}$ mixing ratios in the troposphere is derived for the MOGUNTIA configuration compared to Spivakovsky et al. (2000). The $\mathrm{NH} / \mathrm{SH}$ ratios are calculated as $\sim 1.37$ and $\sim 1.35$ for the MOGUNTIA and mCB05(KPP) configuration, respectively, being on the high end of other modeling estimates, such as the multi-model estimate of an $\mathrm{NH} / \mathrm{SH}$ ratio of $1.28 \pm 0.10$ by Naik et al. (2013) and the 1.20 ratio reported by Lelieveld et al. (2016).

\subsection{Ozone $\left(\mathrm{O}_{3}\right)$}

The evaluation of modeled $\mathrm{O}_{3}$ mixing ratios against surface observations for the three simulations for the year 2006 is presented in Fig. 5. The seasonal cycle across surface stations is generally well captured by all model configurations for most of the cases. TM5-MP, however, generally overestimates $\mathrm{O}_{3}$ mixing ratios at most $\mathrm{NH}$ sites and for all model configurations, as can be seen, for example, at the Barrow (Fig. 5a) and Mace Head (Fig. 5b) stations, especially during the summer (June-July-August, JJA) season when $\mathrm{O}_{3}$ is overestimated by about 8 and $3 \mathrm{ppb}$, respectively. However, at Víznar (Spain) and Mauna Loa (USA) (Fig. 5c and $\mathrm{d}$, respectively), model results are closer to the observed $\mathrm{O}_{3}$ mixing ratios, showing overall lower biases (i.e., $\sim 1-$ $3 \mathrm{ppb}$ ). In the $\mathrm{SH}$ (except for the polar circle), the model simulates the seasonal cycle of the $\mathrm{O}_{3}$ surface mixing ratios well but with average positive biases of $\sim 6-10 \mathrm{ppb}$ in Cape Point (South Africa) and Baring Head (New Zealand) (Fig. 5e, f). At the South Pole (USA) and Syowa (Japan) stations in Antarctica (Figs. 5g, h), the model also captures the observed seasonality well $(R=\sim 0.9)$, except for a neg- 
(a)

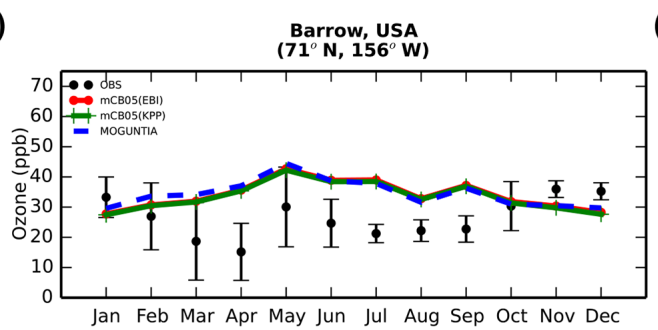

(c)

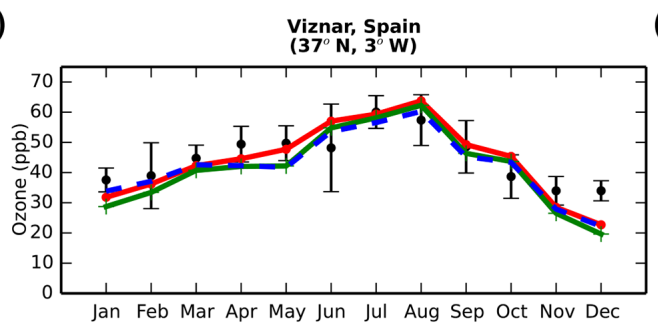

(e)

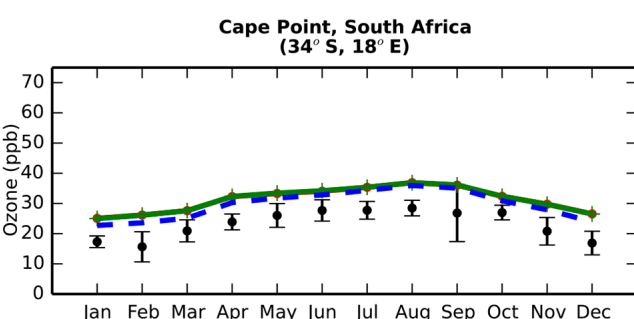

(g)

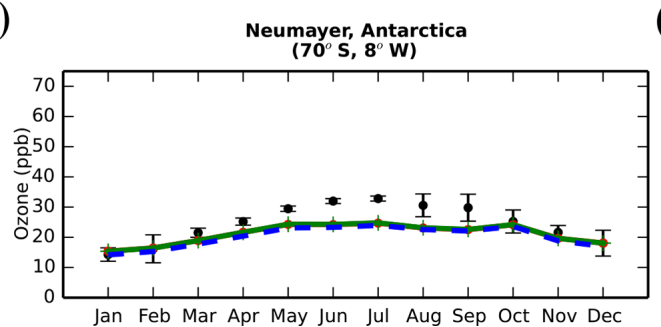

(b)

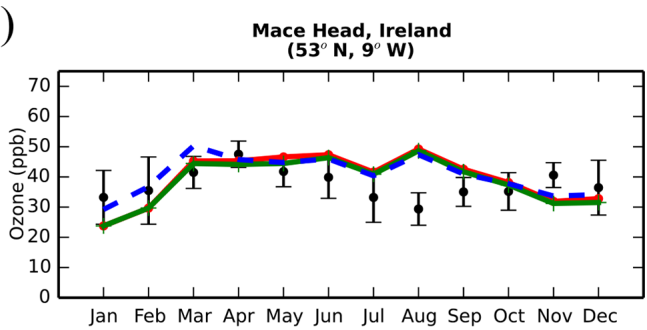

(d)

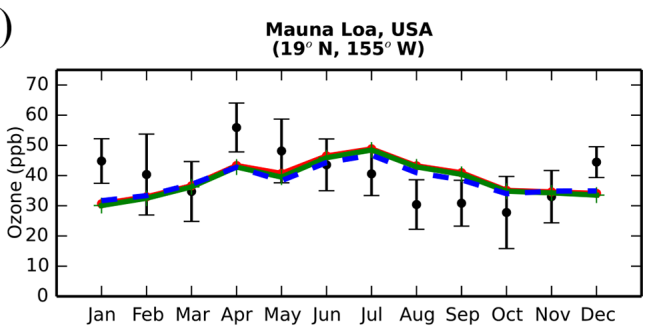

(f)

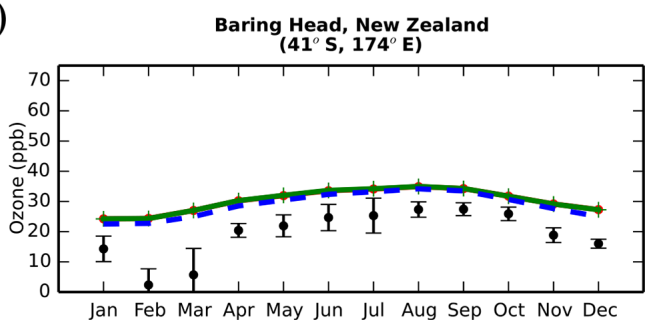

(h)

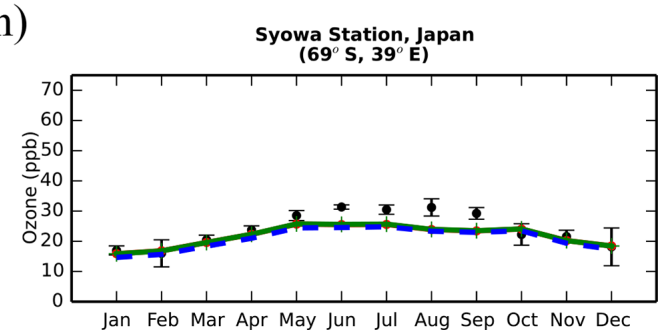

Figure 5. Monthly mean comparison of TM5-MP surface $\mathrm{O}_{3}(\mathrm{ppb})$ against surface observations (black line) from EMEP and WOUDC databases for the two chemistry schemes, mCB05(KPP) (green line) and MOGUNTIA (blue line), using colocated model output for 2006 sampled at the measurement times; error bars indicate the standard deviation in the monthly means. For comparison, model results of mCB05 with the EBI solver (red line) are also presented.

ative bias of $\sim 3 \mathrm{ppb}$ during the local winter season. Focusing further on the chemistry mechanisms applied in the model, slightly better consistency is achieved for the MOGUNTIA chemistry scheme in most of the cases. For the mCB05 chemistry scheme, the choice of the solver does not result in any notable difference in simulated surface $\mathrm{O}_{3}$ mixing ratios. Considering all surface $\mathrm{O}_{3}$ observations available for the year 2006 (Fig. S3), the MOGUNTIA chemistry configuration tends to overestimate the available observations with a mean bias of $\sim 6.5 \mathrm{ppb}$. Note that although the differences between the chemistry configurations for surface $\mathrm{O}_{3}$ are small, the mCB05(KPP) configuration shows the lowest bias $(\sim 5.2 \mathrm{ppb})$, whereas the mCB05(EBI) bias is closer to that of the MOGUNTIA configuration $(\sim 6.1 \mathrm{ppb})$.
Ozonesonde observations are used to evaluate the models' ability to reproduce the $\mathrm{O}_{3}$ vertical profiles. Indicatively, Fig. 6 presents the comparison of model results with ozonesonde observations in 2006 at Hohenpeissenberg in Germany and at Macquarie Island in the southwestern $\mathrm{Pa}$ cific Ocean at five pressure levels $(900,800,500,400$, and $200 \mathrm{hPa}$ ) covering the boundary layer and the low and high free troposphere. For this evaluation, all ozonesonde data have been binned to the 34 model pressure levels (see Sect. 2.5). The seasonal cycle at the two stations is well captured by each model configuration. For the highest model levels above $200 \mathrm{hPa}$, all simulations are very close to the measurements, since $\mathrm{O}_{3}$ mixing ratios are mainly determined by the upper boundary condition that is used (see Sect. 2.1). Comparisons for other WOUDC stations around the globe 
(a)
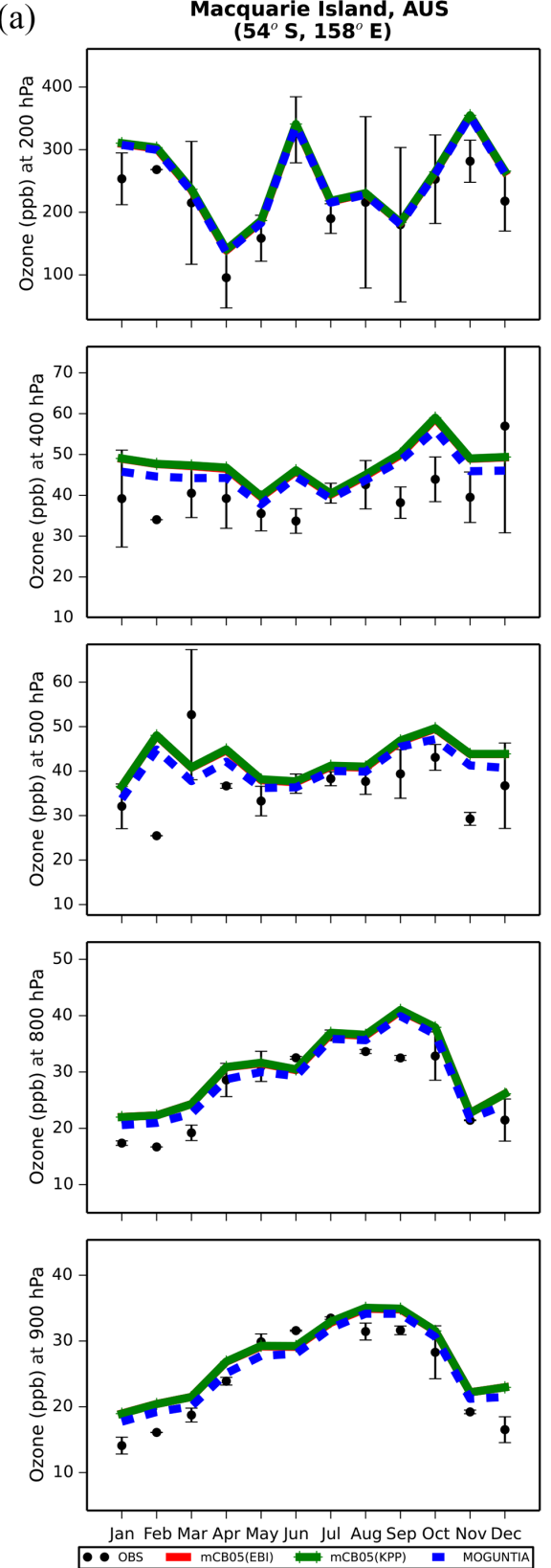

(b)
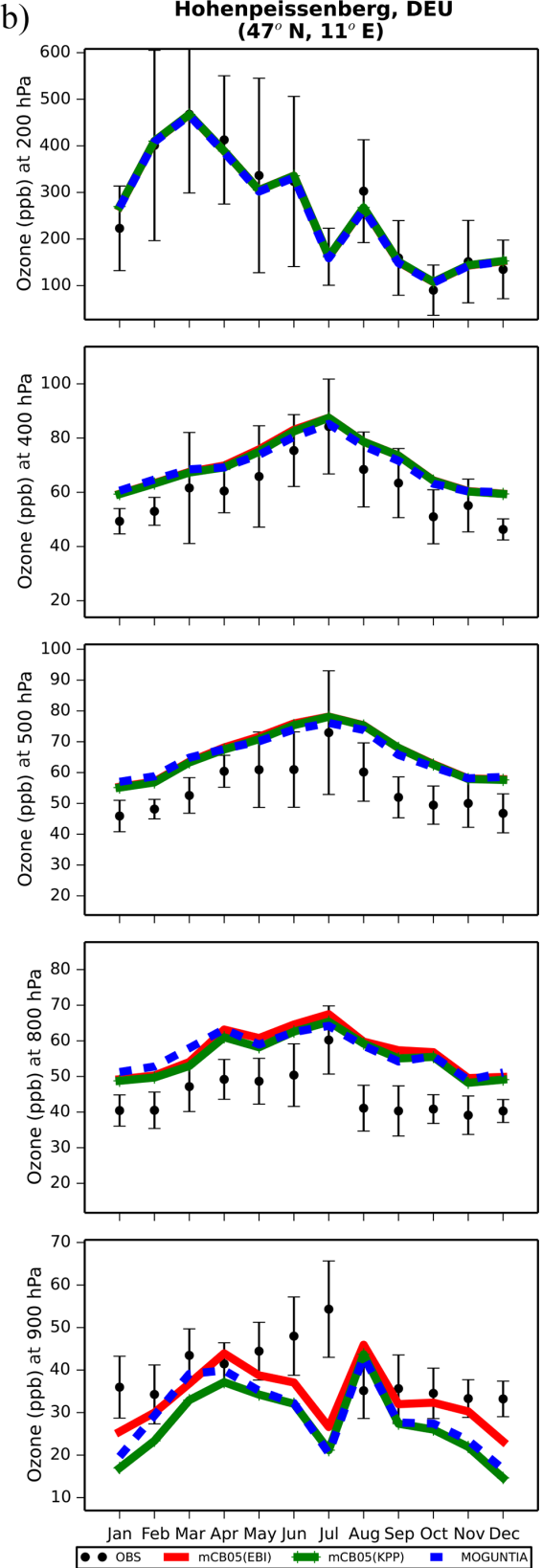

Figure 6. Monthly mean comparison of TM5-MP $\mathrm{O}_{3}(\mathrm{ppb})$ against sonde observations (black dots; mean and standard deviation) at (a) Hohenpeissenberg and (b) Macquarie Island for different pressure levels (900, 800, 500, 400, $200 \mathrm{hPa}$ ) for the two chemistry schemes, mCB05(KPP) (green line) and MOGUNTIA (blue line), using colocated model output for 2006 sampled at the measurement times; error bars indicate the standard deviation in the monthly means. For comparison, the results of mCB05 with the EBI solver (red line) are also presented.

for the year 2006 are presented in the Supplement (Fig. S4). Overall, all model simulations capture the $\mathrm{O}_{3}$ distribution quite well at almost all sites in the lower troposphere. The MOGUNTIA scheme shows slightly better agreement with observations than the mCB05 configurations, with smaller biases in most of the cases, especially at lower levels (i.e., from $\sim 900 \mathrm{hPa}$ and up to $\sim 500 \mathrm{hPa}$ ). Concerning the impact of the chemistry solver, the vertical $\mathrm{O}_{3}$ concentration simulated using the mCB05 mechanism shows no notable differences between the use of KPP and EBI in most of the cases. Overall, considering all available ozonesonde data for the year 2006 (Fig. S4), the MOGUNTIA chemistry in TM5-MP results in an overestimation of the ozonesonde observations by roughly $16 \%(R=0.96$, BIAS $=4.7 \mathrm{ppb}$, $\mathrm{NME}=15.6 \%$ ), which is slightly smaller compared to the mCB05 chemistry configurations. 
Figure $\mathrm{S} 5$ presents a comparison of $\mathrm{O}_{3}$ mixing ratios in the upper troposphere-lower stratosphere (UTLS) simulated by TM5-MP for the two chemistry configurations (i.e., mCB05(KPP) and MOGUNTIA), with in situ observations from the MOZAIC airborne program (see Sect. 3.1), as a function of latitude. The accuracy of the MOZAIC $\mathrm{O}_{3}$ measurements is $\pm 2 \mathrm{ppb}$ (Marenco et al., 1998). For this comparison, the MOZAIC measurements are binned on the vertical grid of TM5-MP. The model evaluation at pressure levels $<300 \mathrm{hPa}$ indicates there is good agreement of both configurations with the observed mixing ratios. A positive bias in April of the order of $\sim 20 \mathrm{ppb}$ is calculated for the model, but smaller biases are found around the tropics and at latitudes north of $40^{\circ} \mathrm{N}$ (Fig. S5a). In October (Fig. S5b), a constant positive bias of roughly $20 \mathrm{ppb}$ is calculated for both configurations. This could be caused by the limited vertical resolution of this model version in the UTLS region. Note that 34 vertical levels were employed for this study with a higher resolution in the upper troposphere-lower stratosphere region. Part of the model overestimation could also be attributed to systematic errors, as also reported in previous studies (e.g., Huijnen et al., 2010). Possible causes include cumulative effects such as a lack of diurnal or weekly variation in the $\mathrm{NO}_{x}$ emissions from the road transport sector, an underestimation of surface deposition during summer, or errors in the representation of nocturnal boundary layer dynamics (see, e.g., Williams et al., 2012).

\subsection{Carbon monoxide (CO)}

Figure 7 presents the model performance concerning surface $\mathrm{CO}$ mixing ratios by comparing a series of flask observations for the year 2006. CO is underestimated at most sites in the NH for all TM5-MP configurations, e.g., at the Barrow Observatory and Mace Head station (Fig. 7a, b), especially during boreal spring (March-April-May, MAM), by about $30 \mathrm{ppb}$ on average. In the tropics, negative biases $(\sim 16-20 \mathrm{ppb})$ are observed at Mauna Loa and Mahé island (Fig. 7c, d). At other stations in the SH, the model simulates the $\mathrm{CO}$ surface mixing ratios well, with both positive and negative biases depending on the season (Fig. 7e, f). In Antarctica, at the South Pole and Syowa stations (Fig. 7g, h), the model also shows a small positive bias up to $\sim 3$ ppb during the local winter season. The seasonal cycle across stations is generally well captured by all model chemistry configurations (i.e., $R=0.7-0.9$ ). The full set of $\mathrm{CO}$ comparisons with flask data is further presented in the Supplement (Fig. S6). Overall, the MOGUNTIA and mCB05(KPP) configurations underestimate the flask observations for the year 2006 , with a negative bias of around $30 \mathrm{ppb}$ and a correlation coefficient for both configurations of $R=0.45$. Notably, the mCB05(EBI) model configuration tends to produce lower biases in the SH, where emission strengths are in general low, compared to the other two configurations (i.e., approximately -3 vs. -4 and $-5 \mathrm{ppb}$ for mCB05(KPP) and MO-
GUNTIA, respectively). In contrast, the MOGUNTIA chemistry configuration results in lower biases in the $\mathrm{NH}$ where the majority of anthropogenic emissions occur (i.e., approximately -30 vs. -31 and $-33 \mathrm{ppb}$ for mCB05(EBI) and mCB05(KPP), respectively).

Total CO columns from the MOGUNTIA and mCB05(KPP) model configurations are compared to the total column densities retrieved from the MOPITT satellite instrument (Deeter et al., 2013, 2019; Ziskin, 2019) for the year 2006 (Fig. 8). Co-sampling with averaging kernels has been applied to the modeled $\mathrm{CO}$ concentration profiles (i.e., in the same manner as for $\mathrm{NO}_{2}$; see Sect. 5.1). Note that when the absolute difference in surface pressure between the MOPITT retrieval and the TM5-MP simulation is larger than $5 \mathrm{hPa}$, the measurements were excluded from the comparison. For the MOGUNTIA configuration, the model shows a mean underestimation of $-8.54 \times 10^{16}(R=0.82)$ and $-1.18 \times 10^{17}$ molec. $\mathrm{cm}^{-2}(R=0.91)$ compared to daily and annual averages of MOPITT data, respectively. However, the correlation is slightly improved compared to the $\mathrm{mCB} 05(\mathrm{KPP})$ configuration $(R=0.78$ and $R=0.88$ for daily and annual values, respectively). As in the comparison with surface data, the biases in total column $\mathrm{CO}$ in the MOGUNTIA and mCB05(KPP) configurations deteriorated compared to the mCB05(EBI) configuration, although biases are still small $(\sim-5 \%$ and $\sim-7 \%$ for daily and annual values, respectively). As this pattern can be seen in both KPP configurations, this difference seems to be caused by the implementation of the more accurate Rosenbrock solver. An overview of the statistical comparison of the three model configurations against MOPITT CO measurements is given in Fig. S1b.

Figure S5 further presents the comparison of $\mathrm{CO}$ mixing ratios in the upper troposphere-lower stratosphere (UTLS) simulated by TM5-MP with in situ measurements from the MOZAIC airborne program (see Sect. 3.1). Model evaluation at pressure levels $<300 \mathrm{hPa}$ shows a good correlation for both configurations in the $\mathrm{SH}$, with a small positive bias (up to $\sim 20 \mathrm{ppb}$ ) for the mCB05(KPP) configuration in April around the Equator and a small negative bias $(\sim 10 \mathrm{ppb})$ for the MOGUNTIA configuration for latitudes below $10^{\circ} \mathrm{N}$. Both configurations present a strong negative bias $(\sim 30 \mathrm{ppb})$ for latitudes above $20^{\circ} \mathrm{N}$ (Fig. S5c). In October (Fig. S5d), both the mCB05(KPP) and MOGUNTIA configurations tend to underestimate the observations, with a negative bias of $\sim 20 \mathrm{ppb}$, except for a small positive bias between 0 and $20^{\circ} \mathrm{N}$. This positive model bias in the UTLS could point to a stronger convective uplift (e.g., Krol et al., 2018) in tropical Africa in April or to possible misrepresentations of biomass burning emission strengths and horizontal and vertical distributions (e.g., Daskalakis et al., 2015; Nechita-Banda et al., 2018). Indeed, MOZAIC data show an increase in CO mixing ratios from the $\mathrm{NH}$ (April) to the $\mathrm{SH}$ (October), mainly due to the impact of biomass burning processes. Overall, the model configurations in this work present both positive and 
(a)

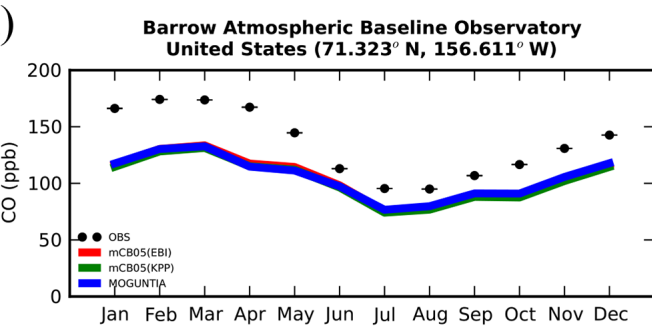

(c)

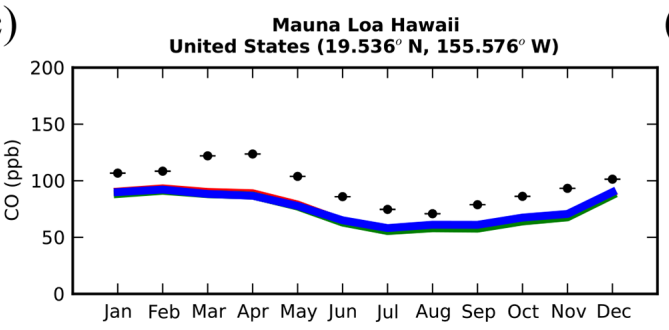

(e)

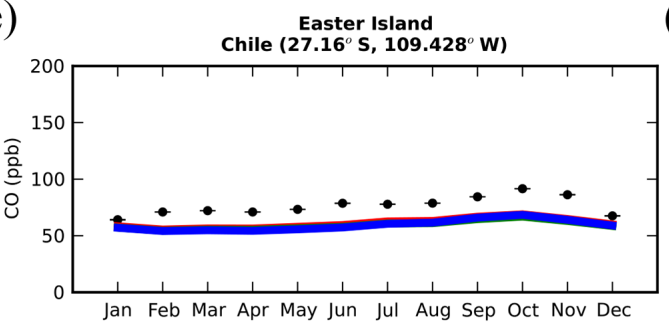

(g)

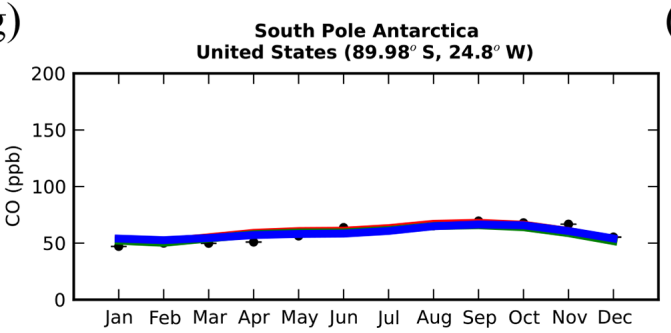

(b)

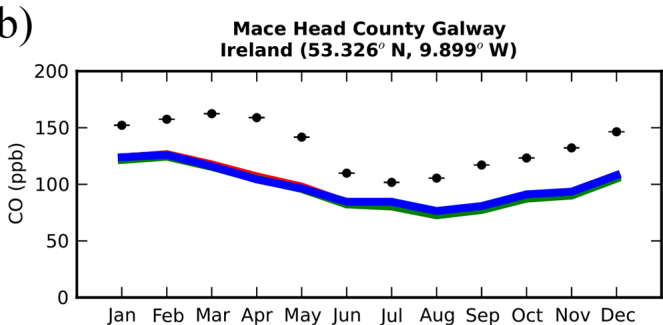

(d)

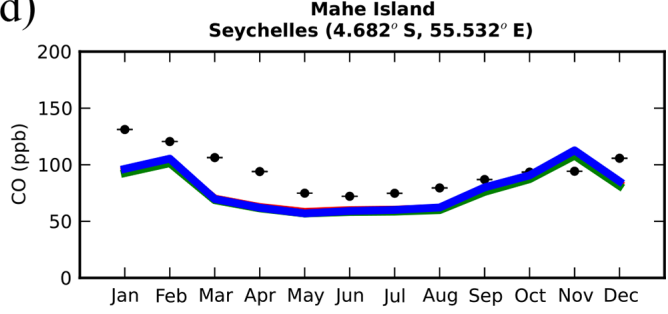

(f)

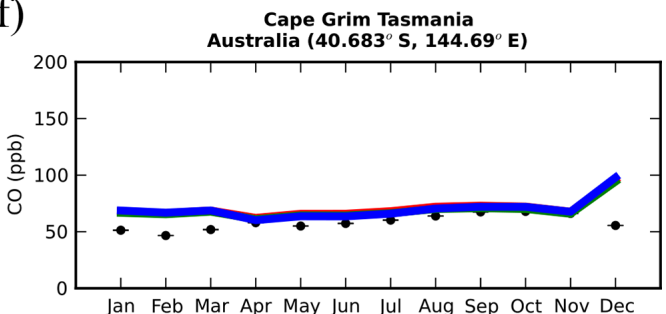

(h)

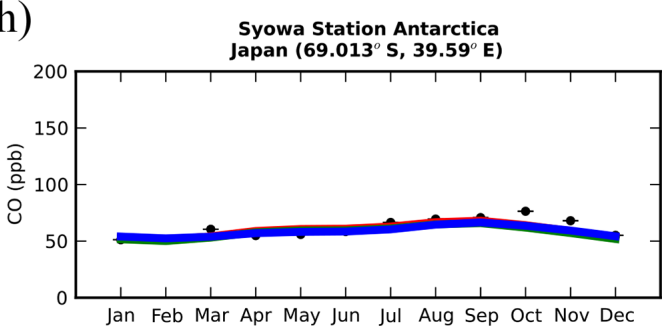

Figure 7. Monthly mean comparison of TM5-MP surface CO (ppb) against flask measurements (black line) for the two chemistry schemes, mCB05(KPP) (green line) and MOGUNTIA (blue line), using colocated model output for 2006 sampled at the measurement times; error bars indicate the standard deviation in the monthly means. For comparison, model results of mCB05 with the EBI solver (red line) are also presented.

negative biases compared to the MOZAIC observations, with observations indicating larger latitudinal $\mathrm{CO}$ variability than simulated.

\subsection{Volatile organic compounds (VOCs)}

\subsubsection{Ethane and propane}

Ethane $\left(\mathrm{C}_{2} \mathrm{H}_{6}\right)$ is the lightest alkane, with emissions primarily of anthropogenic origin associated mainly with fossil fuel extraction and use. In the model, the global ethane emission is $11 \mathrm{Tg} \mathrm{yr}^{-1}$ (Table 3), with an atmospheric lifetime of about $56 \mathrm{~d}$ for all chemistry configurations, in close agreement with other studies (e.g., Hodnebrog et al., 2018). Flask measurements indicate that $\mathrm{C}_{2} \mathrm{H}_{6}$ surface mixing ratios are strongly underestimated by all configurations at Mace Head (Fig. 9a) by $\sim 80 \%$, mainly during the winter, also indicating an opposite annual cycle. The latter can be attributed to the misinterpretation of seasonal variation in anthropogenic emissions and/or to $\mathrm{C}_{2} \mathrm{H}_{6}$ oxidation by $\mathrm{OH}$ radicals in the model. Significant underestimations are also observed in the tropics at Mauna Loa, Hawaii (Fig. 9c), of roughly $98 \%(R \approx-0.5)$. In contrast, at Cape Grim, Australia (Fig. 9e), the model is better at reproducing the measured $\mathrm{C}_{2} \mathrm{H}_{6}$ mixing ratios for all configurations, with a higher correlation coefficient $(R=0.5)$ and an NME of around $63 \%$.

The underestimation of the $\mathrm{C}_{2} \mathrm{H}_{6}$ mixing ratio likely indicates that the model lacks primary emissions of $\mathrm{C}_{2} \mathrm{H}_{6}$ and can thus better reproduce atmospheric observations in the $\mathrm{SH}$ where the anthropogenic emissions are not as strong as in the 
MOGUNTIA

(a)

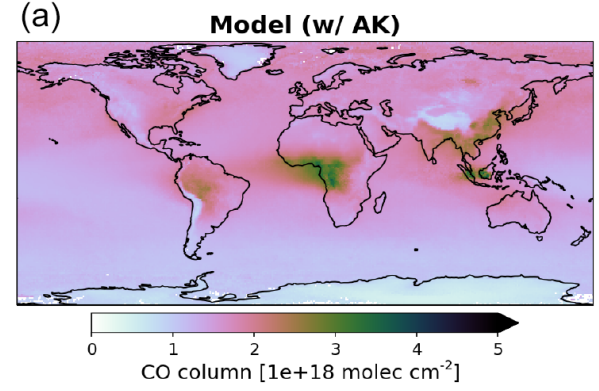

(c)

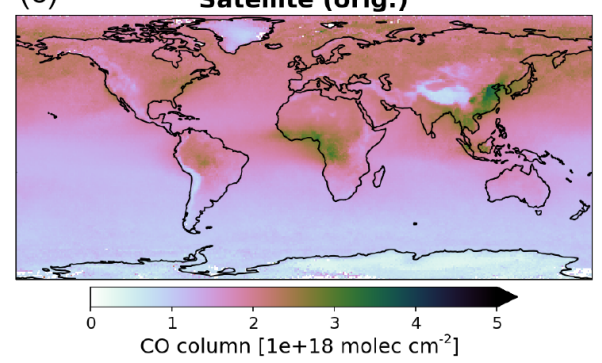

(e)

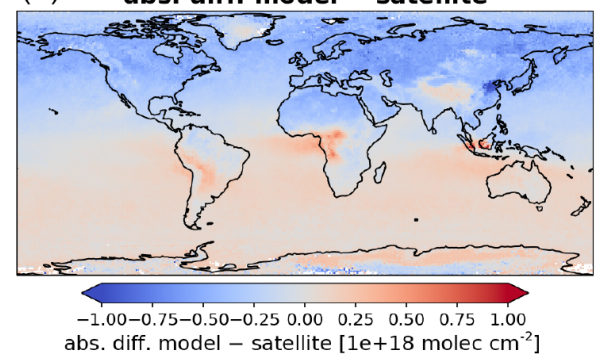

(g)

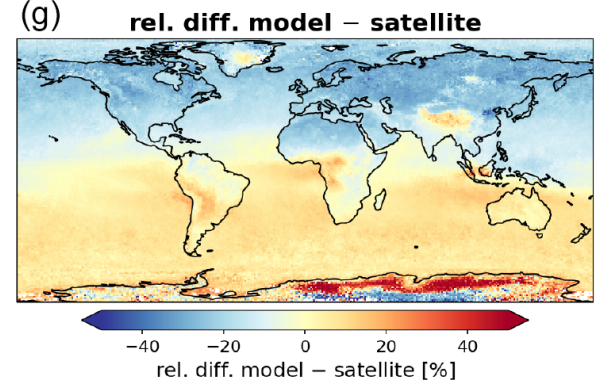

$\mathrm{mCB} 05(\mathrm{KPP})$

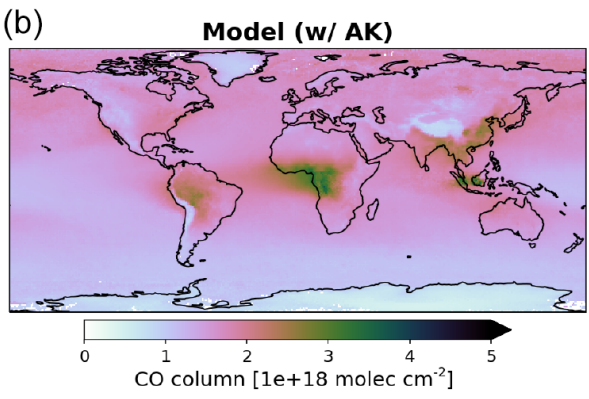

(d)
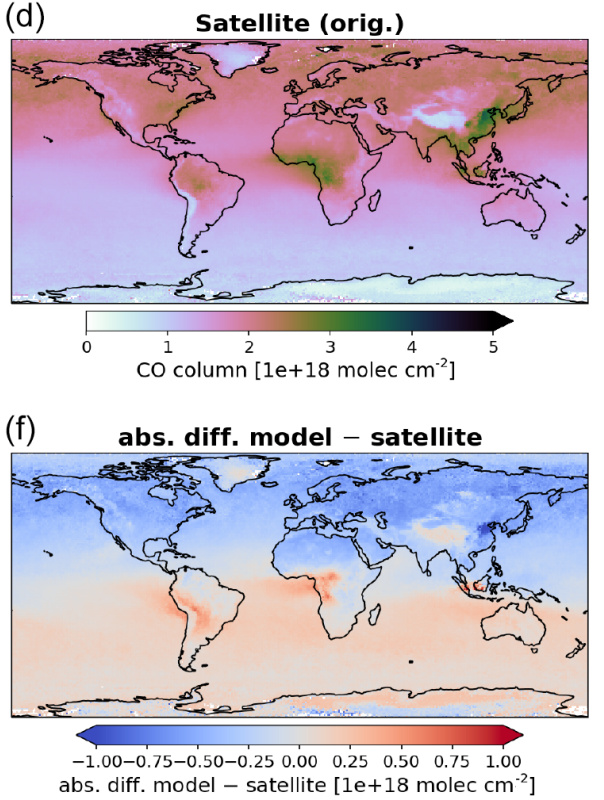

(h)

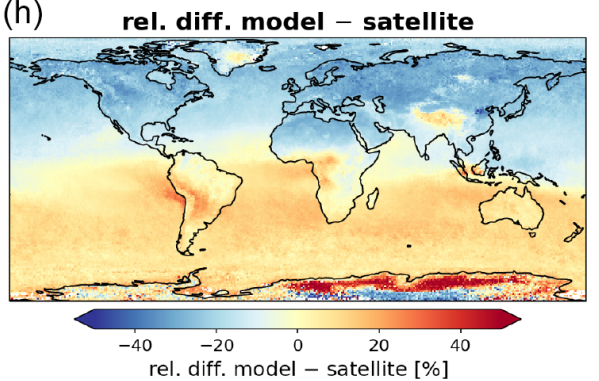

Figure 8. Annual mean comparison of total CO vertical columns $\left(\right.$ molec. $\left.\mathrm{cm}^{-2}\right)$ for the two chemistry schemes of TM5-MP, MOGUNTIA and $\mathrm{mCB} 05(\mathrm{KPP})(\mathbf{a}, \mathbf{b})$ against MOPITT satellite data (c, d), using the respective averaging kernel information for 2006. The absolute (e, f) and relative $(\mathbf{g}, \mathbf{h})$ differences are also presented.

NH. Dalsøren et al. (2018) showed recently that an increase in natural and anthropogenic fossil fuel emissions by a factor of 2 to 3 may significantly improve the simulated $\mathrm{C}_{2} \mathrm{H}_{6}$ and $\mathrm{C}_{3} \mathrm{H}_{8}$ mixing ratios compared to observations. Note that this increase in emissions would result in source estimates close to those calculated by the first global 2-D modeling study of these two hydrocarbons by Kanakidou et al. (1991). To investigate how the model responds to an increase in ethane emis- sions, sensitivity simulations with the MOGUNTIA configuration are performed here by (1) doubling and (2) quadrupling the anthropogenic $\mathrm{C}_{2} \mathrm{H}_{6}$ fossil fuel emissions, resulting in total $\mathrm{C}_{2} \mathrm{H}_{6}$ emissions of $\sim 17.1$ and $\sim 29.5 \mathrm{Tg} \mathrm{yr}^{-1}$, respectively. The global tropospheric burdens have been also increased by a factor of $\sim 1.4$ and 2.2 , respectively. The comparison, however, with flask data (Fig. S7) indicates that the increase in $\mathrm{C}_{2} \mathrm{H}_{6}$ anthropogenic emissions does not signifi- 
(a)

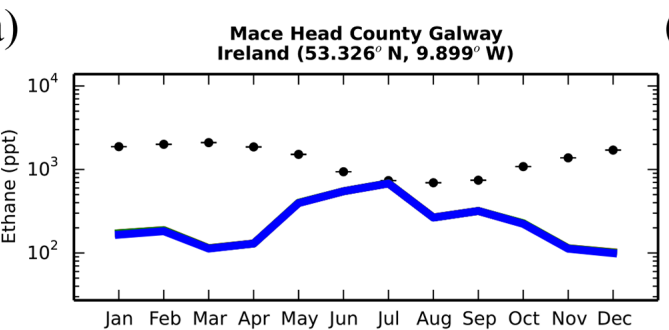

(c)

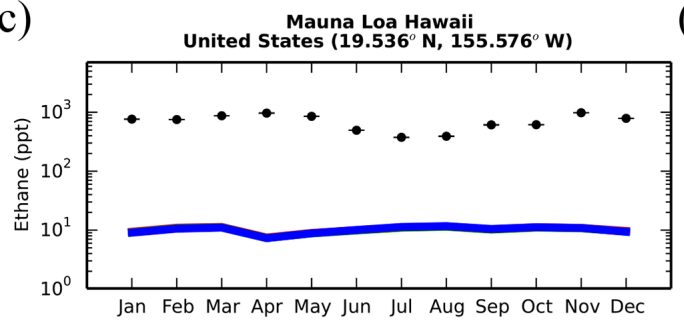

(e)

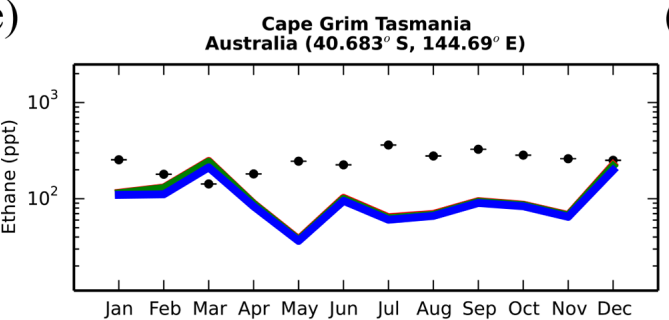

(g)

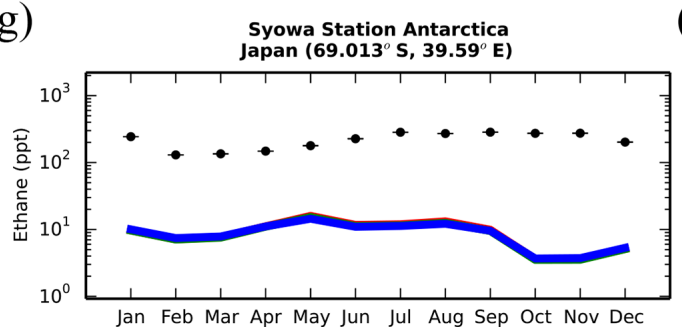

(b)

Mace Head County Galway
Ireland $\left(53.326^{\circ} \mathrm{N}, 9.899^{\circ} \mathrm{W}\right)$

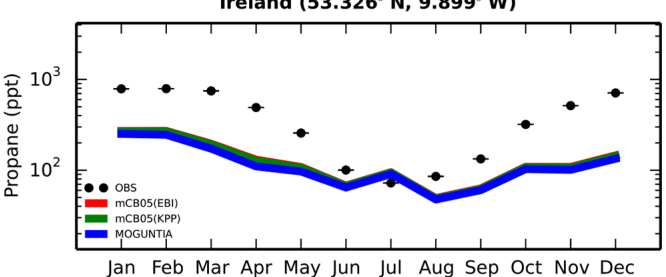

(d) Mauna Loa Hawaii

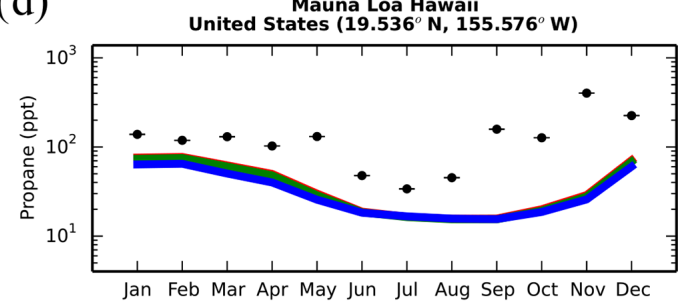

(f)

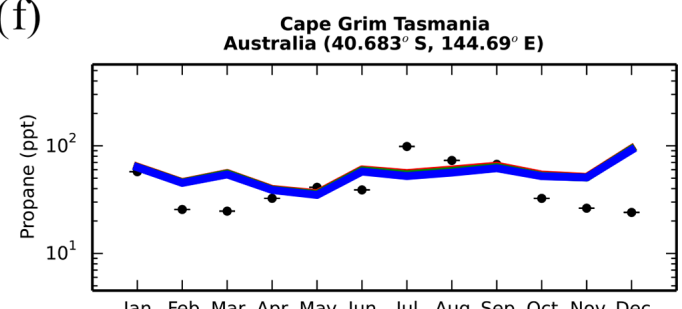

(h)

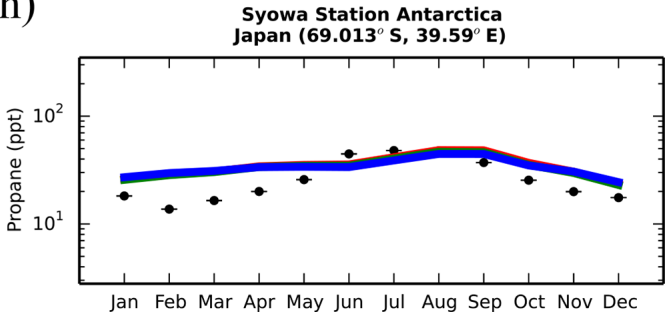

Figure 9. Monthly mean comparison of TM5-MP surface $\mathrm{C}_{2} \mathrm{H}_{6}$ (left column) and $\mathrm{C}_{3} \mathrm{H}_{8}$ (right column) against flask measurements (black dots; ppt) for the two chemistry schemes, mCB05(KPP) (green line) and MOGUNTIA (blue line), using colocated model output for 2006 sampled at the measurement times; error bars indicate the standard deviation in the monthly means. For comparison, model results of mCB05 with the EBI solver (red line) are also presented.

cantly affect the simulated mixing ratios in the model at these specific stations. Overall, this means that even a more aggressive increase in emissions (at least over specific regions) is required, other missing sources need to be considered in the model, or the oxidation of $\mathrm{C}_{2} \mathrm{H}_{6}$ is too fast in the model. The full set of $\mathrm{C}_{2} \mathrm{H}_{6}$ comparisons with flask data is presented in the Supplement (Fig. S8).

Propane $\left(\mathrm{C}_{3} \mathrm{H}_{8}\right)$ is also emitted mainly from anthropogenic sources, and in the current simulations the total emission is $8.5 \mathrm{Tg} \mathrm{yr}^{-1}$ (Table 3), which is lower compared to other reported emission estimates of $\sim 15 \mathrm{Tg} \mathrm{yr}^{-1}$ (Jacob et al., 2002). Model comparison with flask observations (Fig. 9) shows that the model tends to underestimate the measured mixing ratios for all simulations but with higher correlation coefficients compared to $\mathrm{C}_{2} \mathrm{H}_{6}$ in most of the cases. $\mathrm{C}_{3} \mathrm{H}_{8}$ is underestimated in the $\mathrm{NH}$ at Mace Head (Fig. 9b) during the winter and autumn seasons by $72 \%-74 \%$. In the tropics, strong negative biases of $\sim 100 \mathrm{ppt}$ are observed at Mauna Loa (Fig. 9d). However, the model simulates the $\mathrm{C}_{3} \mathrm{H}_{8}$ surface mixing ratios better in the $\mathrm{SH}$ at Cape Grim compared to stations in the $\mathrm{NH}$ (Fig. 9b, d, f) due to the weaker impact of anthropogenic emissions. In contrast to the $\mathrm{C}_{2} \mathrm{H}_{6}$ evaluation, however, the model satisfactorily simulates the observed $\mathrm{C}_{3} \mathrm{H}_{8}$ mixing ratios at the South Pole (Fig. 9h), with a small overestimation during the local summer season. The full set of $\mathrm{C}_{3} \mathrm{H}_{8}$ comparisons with flask data is presented in Fig. S9. As for the case of $\mathrm{C}_{2} \mathrm{H}_{6}$, to further investigate the impact of emissions on the simulated $\mathrm{C}_{3} \mathrm{H}_{8}$ mixing ratios, additional simulations are performed by (1) doubling and (2) quadrupling the anthropogenic fossil fuel emissions, resulting in 
(a)

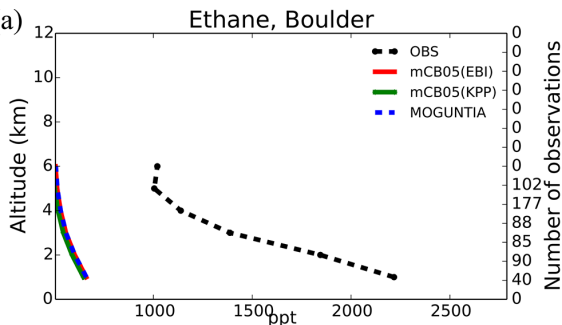

(c)

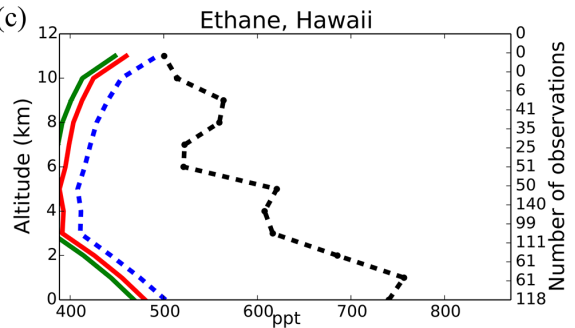

(e)

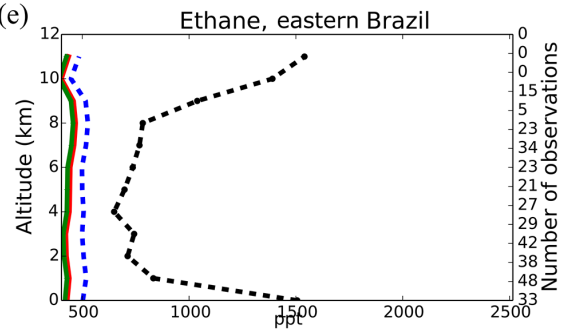

(g)

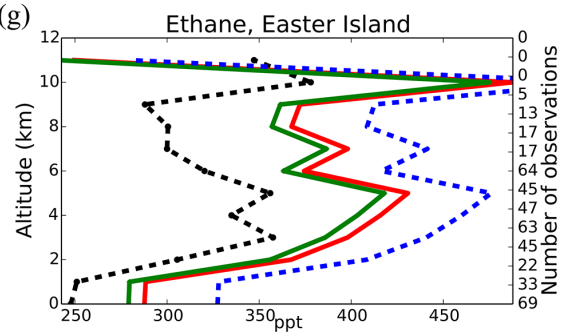

(b)

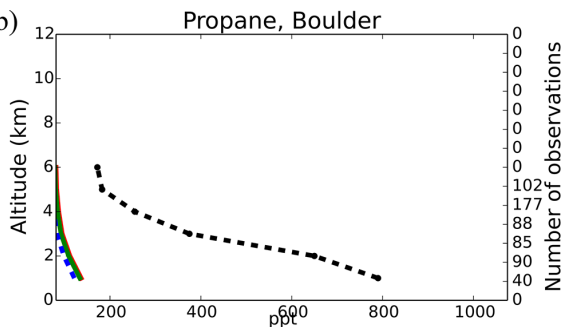

(d)

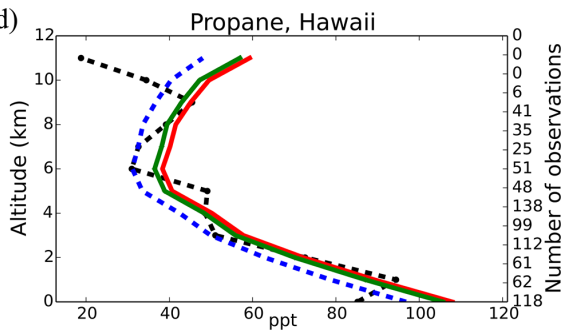

(f)

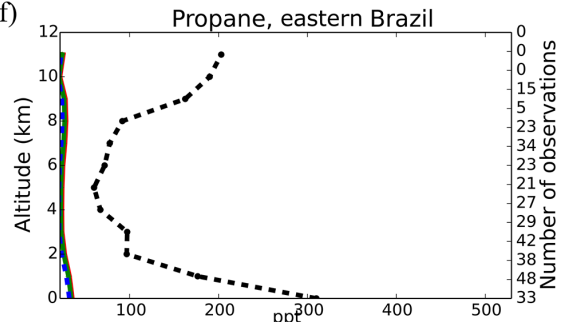

(h)

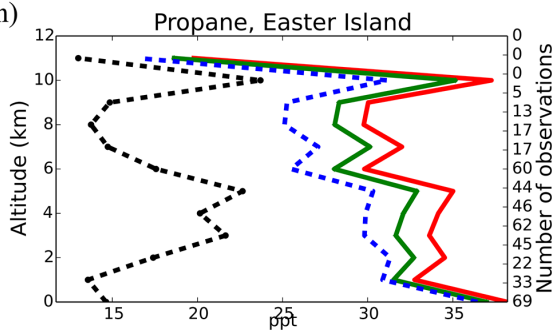

Figure 10. Comparison of TM5-MP vertical profiles $(\mathrm{km})$ of $\mathrm{C}_{2} \mathrm{H}_{6}$ (left column) and $\mathrm{C}_{3} \mathrm{H}_{8}$ (right column) against aircraft observations (black line; ppt) for the two chemistry schemes, mCB05(KPP) (green line) and MOGUNTIA (blue line), using colocated model output for 2006 sampled at the measurement times; error bars indicate the standard deviation. For comparison, model results of mCB05 with the EBI solver (red line) are also presented. The numbers on the right vertical axis indicate the number of available measurements.

total $\mathrm{C}_{3} \mathrm{H}_{8}$ emissions of $\sim 14.9$ and $\sim 27.9 \mathrm{Tg} \mathrm{yr}^{-1}$, respectively. The global $\mathrm{C}_{3} \mathrm{H}_{8}$ tropospheric burdens have been increased by a factor of $\sim 1.7$ and 3.2, respectively. Figure $\mathrm{S} 7$ indicates that an increase in $\mathrm{C}_{3} \mathrm{H}_{8}$ emissions by 2 times tends to significantly improve the model simulations, whereas a respective increase by 4 times tends to overestimate the observed mixing ratios.

Comparison with $\mathrm{C}_{2} \mathrm{H}_{6}$ and $\mathrm{C}_{3} \mathrm{H}_{8}$ aircraft climatological data (Fig. 10) further indicates that all chemistry configurations tend to underestimate the observed mixing ratios $(\sim 20 \%-60 \%)$ in most of the cases, especially in the upper troposphere. In more detail, in Boulder and eastern Brazil, the model significantly underestimates the observed mixing ratios for both compounds, while in Hawaii $\mathrm{C}_{2} \mathrm{H}_{6}$ is under- estimated but $\mathrm{C}_{3} \mathrm{H}_{8}$ is well simulated by all three configurations. In contrast, at Easter Island, all schemes overestimate the observed mixing ratios for both compounds, although the MOGUNTIA overestimate is larger for $\mathrm{C}_{2} \mathrm{H}_{6}$ and lower for $\mathrm{C}_{3} \mathrm{H}_{8}$ compared to the two mCB05 configurations. The full sets of $\mathrm{C}_{2} \mathrm{H}_{6}$ and $\mathrm{C}_{3} \mathrm{H}_{8}$ comparisons with aircraft climatological data are presented in the Supplement (Figs. S10 and S11, respectively). Overall, considering that the model reasonably simulates the oxidative capacity of the atmosphere, direct emissions are the likely reason for these differences, since both alkanes are oxidized in the troposphere by $\mathrm{OH}$ radicals and no secondary production terms of these alkanes are known. Note, however, that alkane emission fluxes are on the 

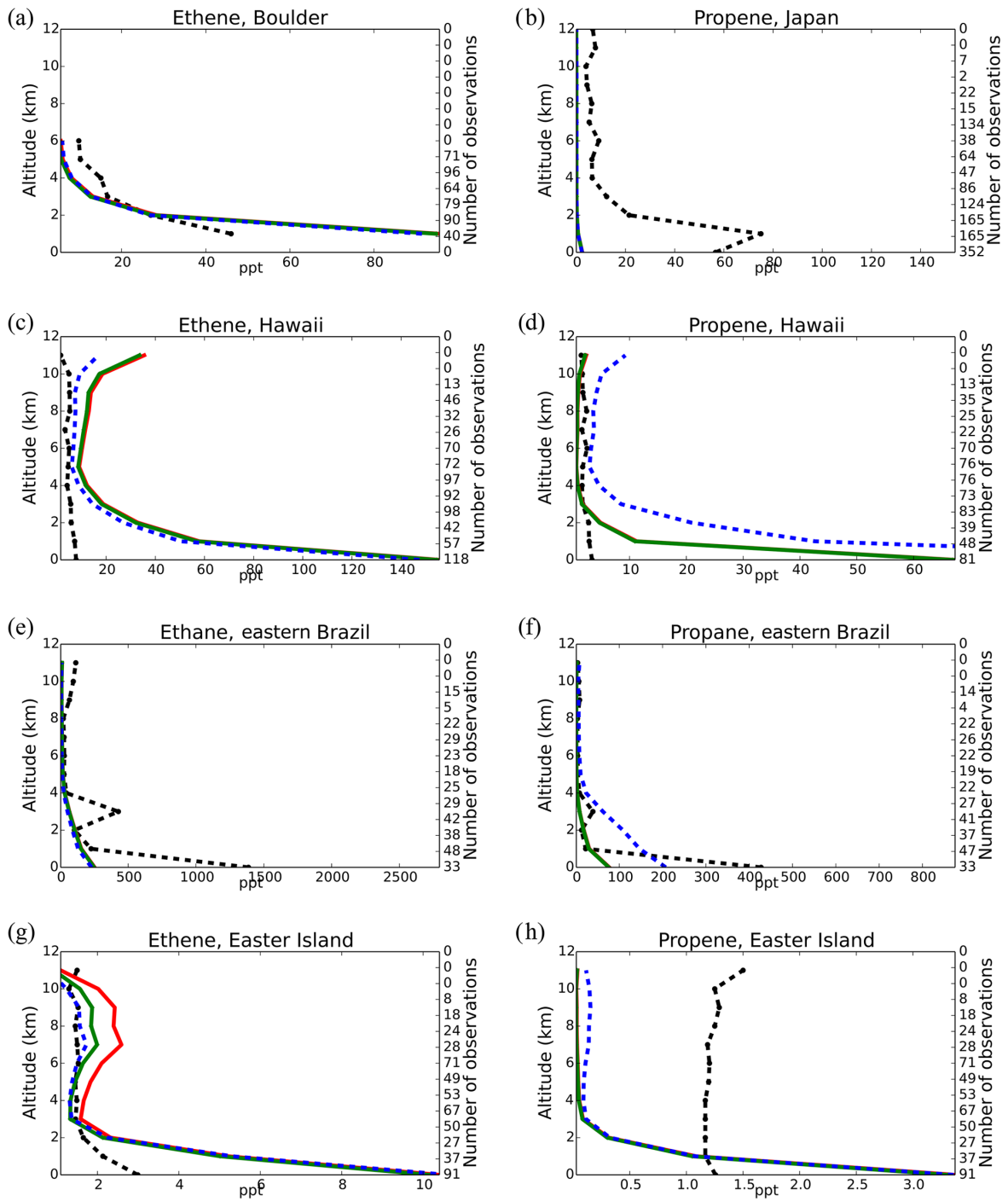

Figure 11. Comparison of TM5-MP vertical profiles $(\mathrm{km})$ of $\mathrm{C}_{2} \mathrm{H}_{4}$ (left column) and $\mathrm{C}_{3} \mathrm{H}_{6}$ (right column) against aircraft observations (black line; ppt) for the two chemistry schemes, mCB05(KPP) (green line) and MOGUNTIA (blue line), using colocated model output for 2006 sampled at the measurement times; error bars indicate the standard deviation. For comparison, model results of mCB05 with the EBI solver (red line) are also presented. The numbers on the right vertical axis indicate the number of available measurements.

low side, as also reported by other studies (e.g., Aydin et al., 2011; Huijnen et al., 2019; Monks et al., 2018).

\subsubsection{Ethene and propene}

Ethene is mainly emitted from biogenic sources and by incomplete combustion from biomass burning, power plants, and combustion engines. $\mathrm{C}_{2} \mathrm{H}_{4}$ emissions in the model are roughly $30 \mathrm{Tg} \mathrm{yr}^{-1}$ (Table 3), close to the estimate of Huijnen et al. (2019) but on the high side compared to the $21 \mathrm{Tg} \mathrm{yr}^{-1}$ reported by Toon et al. (2018). The three chemistry configurations produce similar mixing ratios of $\mathrm{C}_{2} \mathrm{H}_{4}$ in most of the cases. Nevertheless, the comparison with aircraft observations (Fig. 11) indicates underestimated mixing ratios in the upper troposphere. In more detail, the model reproduces ( $R=0.97)$ the vertical distribution of $\mathrm{C}_{2} \mathrm{H}_{4}$ well in Boulder (USA). However, observed mixing ratios close to the surface (up to $\sim 2 \mathrm{~km}$ ) are overestimated by the model, while observations at the higher levels (up to $\sim 6 \mathrm{~km}$ ) are underestimated. In the tropics, the observed mixing ratios in the lower and upper troposphere (e.g., in Hawaii) are slightly overestimated by the model for all configurations, although for the MOGUNTIA configuration this overestimate is the lowest. In remote regions, where the impact of direct emissions is negligible (e.g., at Easter Island), the model overestimates $\mathrm{C}_{2} \mathrm{H}_{4}$ close to the surface $(\sim 1 \mathrm{~km})$, but some negative biases appear aloft. At higher altitudes, however, all configurations overestimate the observed $\mathrm{C}_{2} \mathrm{H}_{4}$ mixing ratios (Fig. $11 \mathrm{~g}$ ), 
but again the MOGUNTIA model configuration better reproduces the observations. Overall, these deviations from the observations could be attributed to (1) the background concentrations not being well resolved by the model, (2) the severe uncertainties in emission fluxes, and (3) chemistry that is not well understood (e.g., Huijnen et al., 2019; Pozzer et al., 2007), such as the $\mathrm{C}_{2} \mathrm{H}_{4}$ production during VOC decomposition in the atmosphere.

Propene $\left(\mathrm{C}_{3} \mathrm{H}_{6}\right)$ emissions in the model are $\sim 32 \mathrm{Tg} \mathrm{yr}^{-1}$ (Table 3). The two mCB05 configurations produce similar $\mathrm{C}_{3} \mathrm{H}_{6}$ mixing ratios, but MOGUNTIA tends to simulate higher values, especially in the tropics, in Hawaii (Fig. 11d), and in eastern Brazil (Fig. 11f). Close to the surface, where the impact of the emissions is stronger, the model severely overestimates observations (Fig. 11d, f), except for Japan (Fig. 11b). For the MOGUNTIA configuration, this overestimation is more substantial in the tropics compared to the mCB05 chemistry scheme. An overestimation of the observed mixing ratio close to the surface is also found in other regions, especially in the $\mathrm{SH}$, such as in eastern Brazil (Fig. 11f), and in remote regions where the direct impact of emissions is negligible, such as Easter Island (Fig. 11h). However, at Easter Island (Fig. 11h), the model fails to reproduce the observed $\mathrm{C}_{3} \mathrm{H}_{6}$ vertical profile, resulting in a significant underestimation of the observed mixing ratios. Overall, even though the evaluation of vertical profiles should be considered here only as a climatological comparison, the reason for the model underestimation of $\mathrm{C}_{3} \mathrm{H}_{6}$ mixing ratios at higher altitudes is likely a combination of the emission strengths, the simulated vertical distribution, and the potential but still unaccounted for secondary production from higher VOC oxidation. All comparisons for $\mathrm{C}_{2} \mathrm{H}_{4}$ and $\mathrm{C}_{3} \mathrm{H}_{6}$ with aircraft climatological data are presented in Figs. S12 and S13, respectively.

\section{Summary and conclusions}

This study documents and evaluates the implementation of the tropospheric chemistry scheme MOGUNTIA in the global chemistry and transport model TM5-MP. The MOGUNTIA scheme is a comprehensive gas-phase chemistry mechanism that explicitly accounts for the oxidation of light hydrocarbons, coupled with an updated representation of isoprene oxidation, along with a simplified representation of terpenes and aromatics chemistry. The newly coupled chemistry scheme in TM5-MP is compared to the existing chemistry scheme of the model, mCB05. Another feature implemented in the TM5-MP chemistry code is the Rosenbrock solver that replaces the classical EBI method. For this, a simple preprocessor directive has been implemented in the model to choose between the two solvers during model compilation. In the case of the Rosenbrock solver, the KPP software has been used to generate the chemistry code coupled with the TM5MP. To further examine the impact of the solver on the TM5-
MP atmospheric simulations and performance, the mCB05 scheme is also tested using the Rosenbrock solver.

Global budgets of $\mathrm{O}_{3}, \mathrm{CO}$, and $\mathrm{OH}$, for all simulations performed for this work, are calculated and compared with estimates published in the literature. In more detail, the $\mathrm{O}_{3}$ budget calculated with the MOGUNTIA chemistry scheme falls within 1 standard deviation of mean estimates from other modeling studies. However, the new MOGUNTIA scheme reduces the tropospheric $\mathrm{O}_{3}$ burden by $\sim 3 \%$ compared to the mCB05 configurations. For tropospheric $\mathrm{CO}$, a respective reduction in the atmospheric lifetime $(\sim 6 \%)$ provides evidence that the implementation of the MOGUNTIA chemistry leads to an increase in the oxidative capacity of the troposphere in TM5-MP. This also holds for the atmospheric $\mathrm{CH}_{4}$ chemical lifetime that is calculated here to be about 8.0 years for the MOGUNTIA chemistry scheme, which is roughly $3 \%-5 \%$ shorter compared to $\mathrm{mCB} 05(\mathrm{KPP})$ and $\mathrm{mCB} 05(\mathrm{EBI})$ configurations.

The large-scale variability in space and time of modeled tropospheric $\mathrm{NO}_{2}, \mathrm{OH}, \mathrm{O}_{3}, \mathrm{CO}$, and light VOCs (i.e., $\mathrm{C}_{2} \mathrm{H}_{6}$, $\mathrm{C}_{2} \mathrm{H}_{4}, \mathrm{C}_{3} \mathrm{H}_{8}, \mathrm{C}_{3} \mathrm{H}_{6}$ ) has been evaluated for the year 2006 and compared to several sets of in situ observations, satellite retrievals, and climatological data. Overall, both the lumpedstructure (i.e., the mCB05) and the lumped-molecule (i.e., the MOGUNTIA) mechanisms appear to be able to satisfactorily represent the tropospheric chemistry. In most of the cases, lower biases compared to measurements are calculated when the MOGUNTIA chemistry configuration is used. The model simulates the major observed features of the spatial and temporal variability well in surface observations for $\mathrm{O}_{3}$ and $\mathrm{CO}$. The observed background surface $\mathrm{O}_{3}$ mixing ratios are captured with a bias of $\sim 6.5 \mathrm{ppb}$ for the MOGUNTIA configuration, very close to the mCB05 configurations. Ozone in the vertical matches on average within $\sim 5 \mathrm{ppb}$ for all configurations, and the model is able to capture the variability observed by ozonesondes well. In contrast, the model underestimates the available $\mathrm{CO}$ flask observations by roughly $30 \%$ for all configurations, most likely linked to uncertainties in the seasonal cycle of anthropogenic emissions and the representation of biomass burning $\mathrm{CO}$ emissions. For the model comparison with observed light VOC mixing ratios, all chemistry configurations clearly show that significant uncertainties still exist regarding their emission strength or poorly understood chemistry, such as secondary chemical production during the decomposition of higher VOCs in the atmosphere. Sensitivity simulations performed indicate that increases in emissions may improve the simulation of the atmospheric mixing ratios of some light VOCs, such as $\mathrm{C}_{3} \mathrm{H}_{8}$. However, our results suggest that changes in emissions should not just be based on fixing the model's emissions using a specific (constant) value but that scientifically accepted methods should be used. Future studies should therefore aim at improving source estimates and a better understanding of the processes that govern the budgets of light VOCs. From a chemistry point of view, it would be interest- 
ing to study the chemical formation pathways from higher VOCs. Inverse modeling or data assimilation studies might also be used to "optimize" the emissions in order to minimize the differences between observations and model simulations.

The presented model configurations result in a benchmark of the TM5-MP tropospheric chemistry version upon which future model improvements may take place. Inherent uncertainties need to be reduced and further work is required, focusing mainly on the most poorly understood chemistryrelated processes. For example, further attention concerns the uncertainties in $\mathrm{NO}-\mathrm{NO}_{2}-\mathrm{O}_{3}$ cycling along with the atmospheric fate of ORGNTRs and their impacts on the oxidative capacity of the troposphere. Attention is also needed for the treatment of aerosols and clouds, in particular ice clouds and their impact on photolysis frequencies. Other issues that need to be resolved are related to the significant uncertainties in light hydrocarbon mixing ratios - as clearly seen in the model comparison to surface and aircraft observations and their potential impact on the oxidative capacity of the troposphere. Considering that both chemistry schemes underestimate light VOC mixing ratios in most of the cases, the use of a more detailed scheme such as MOGUNTIA will allow us to better understand the causes of this deviation compared to the lumped representation of VOC chemistry in the mCB05 mechanism. This is especially relevant over tropical regions with high biogenic VOC emissions under low$\mathrm{NO}_{x}$ conditions. For this, a more dedicated comparison of the model with in situ observations and satellite retrievals is needed. MOGUNTIA also contains an ample number of oxygenated VOCs that are observed in the atmosphere at significant levels and further involved in aerosol formation, making the scheme appropriate for detailed studies. On top of this, the implementation of the KPP software in the model makes the code a lot more flexible for chemistry updates compared to the previous EBI-based chemistry versions. The use of the KPP in TM5-MP reduces the uncertainties in solving stiff chemistry equations and opens up new possibilities for model development, such as the construction of an adjoint of the chemistry mechanism that can be used in 4DVar data assimilation systems (e.g., Henze et al., 2007). Another possible application is to more accurately explore atmospheric chemistry-climate interactions, since TM5-MP is also coupled to the Earth System Model EC-Earth (e.g., Van Noije et al., 2014). Note, however, that despite the clear benefits regarding code development and management, the use of a more sophisticated solver such as the Rosenbrock solver and the implementation of a detailed chemistry scheme such as MOGUNTIA make the code computationally more expensive. Overall, this work shows that the newly coupled chemistry version of TM5-MP works as well as - or better than in some cases - the previous chemistry versions of the model, opening opportunities for further chemistry developments and more detailed tropospheric investigations by the TM and EC-Earth communities.
Code availability. The TM5-MP code used for this study can be downloaded from Zenodo (https://doi.org/10.5281/zenodo.3759200, Myriokefalitakis et al., 2020); a request to generate a new user account for access to the SVN server hosted at KNMI, the Netherlands, can be made by emailing Philippe Le Sager (sager@knmi.nl). Any new user groups need to agree to the protocol set out for use, and it is expected that any developments will be accessible to all users after the publication of results. Attendance at 9-monthly TM5 international meetings is encouraged to avoid duplicity and conflict of interests.

Supplement. The supplement related to this article is available online at: https://doi.org/10.5194/gmd-13-5507-2020-supplement.

Author contributions. This paper resulted from the deliberations of the 27th International TM5 Meeting, 28-29 June 2018, Utrecht, the Netherlands (SM, MCK, TvN, PLS, SH, ND, MK). SM and MCK developed the chemistry code coupled to the model. SM and MK provided the original chemistry scheme equations. JEW developed both the photolysis code and mCB05 chemical mechanism, including the implementation of updated photolysis frequencies for the additional organics included in the MOGUNTIA chemistry scheme. AG contributed to reaction data updates and coupling. AH developed and provided model evaluation tools with satellite retrievals. $\mathrm{VH}$ provided model evaluation tools and a collection of observation data. SM, ND, AH, and PLS performed the model evaluation. SM wrote the paper, and all authors contributed to the preparation of the paper.

Competing interests. The authors declare that they have no conflict of interest.

Acknowledgements. Stelios Myriokefalitakis acknowledges financial support for this research from the European Union's Horizon 2020 research and innovation program under Marie SkłodowskaCurie grant agreement no. 705652 - ODEON. Maarten C. Krol is supported by the European Research Council (ERC) under the European Union's Horizon 2020 research and innovation program under grant agreement no. 742798 - COS-OCS. Stelios Myriokefalitakis and Angelos Gkouvousis acknowledge financial support from the National Observatory of Athens research grant (no. 5065). Maria Kanakidou and Nikos Daskalakis acknowledge funding by the Deutsche Forschungsgemeinschaft (DFG, German Research Foundation) under Germany's Excellence Strategy (University Allowance, EXC 2077, University of Bremen). This work was supported by computational time granted from the National Infrastructures for Research and Technology S.A. (GRNET S.A.) in the National HPC facility - ARIS - under project ID PR008001-ADIOS. Model development was carried out on the GRNET HPC ARIS, the Dutch national e-infrastructure, with the support of the SURF Cooperative, and the ECMWF CRAY XC40 high-performance computer facility. Model simulations were performed at the GRNET HPC ARIS and the AETHER HPC cluster at the University of Bremen, funded by the DFG within the scope of the Excellence Initiative. MOPITT CO data were obtained from the NASA Langley Re- 
search Center Atmospheric Science Data Center. $\mathrm{NO}_{2}$ and $\mathrm{HCHO}$ satellite data from OMI and SCIAMACHY were produced in the scope of the European FP7 project QA4ECV (grant no. 6007405). The authors thank two anonymous reviewers for their comments that significantly helped to improve the final paper. This paper is dedicated to the memory of Andreas Hilboll.

Financial support. This research has been supported by the European Research Council (COS-OCS, grant no. 742798) and the H2020 Marie Skłodowska-Curie Actions (ODEON, grant no. 705652). This project has received funding from the European Research Council (ERC) under the European Union's Horizon 2020 research and innovation program under grant agreement no. 742798 .

Review statement. This paper was edited by Rolf Sander and reviewed by two anonymous referees.

\section{References}

Aan de Brugh, J. M. J., Schaap, M., Vignati, E., Dentener, F., Kahnert, M., Sofiev, M., Huijnen, V., and Krol, M. C.: The European aerosol budget in 2006, Atmos. Chem. Phys., 11, 1117-1139, https://doi.org/10.5194/acp-11-1117-2011, 2011.

Andres, R. J. and Kasgnoc, A. D.: A time-averaged inventory of subaerial volcanic sulfur emissions, J. Geophys. Res., 103, 25251, https://doi.org/10.1029/98JD02091, 1998.

Atkinson, R.: Gas-Phase Tropospheric Chemistry of Volatile Organic Compounds: 1. Alkanes and Alkenes, J. Phys. Chem. Ref. Data, 26, 215-290, https://doi.org/10.1063/1.556012, 1997.

Atkinson, R.: Atmospheric chemistry of VOCs and $\mathrm{NO}_{x}$, Atmos. Environ., 34, 2063-2101, https://doi.org/10.1016/S13522310(99)00460-4, 2000.

Atkinson, R., Baulch, D. L., Cox, R. A., Crowley, J. N., Hampson, R. F., Hynes, R. G., Jenkin, M. E., Rossi, M. J., and Troe, J.: Evaluated kinetic and photochemical data for atmospheric chemistry: Volume $\mathrm{I}-$ gas phase reactions of $\mathrm{O}_{x}, \mathrm{HO}_{x}$, $\mathrm{NO}_{x}$ and $\mathrm{SO}_{x}$ species, Atmos. Chem. Phys., 4, 1461-1738, https://doi.org/10.5194/acp-4-1461-2004, 2004.

Aydin, M., Verhulst, K. R., Saltzman, E. S., Battle, M. O., Montzka, S. A., Blake, D. R., Tang, Q. and Prather, M. J.: Recent decreases in fossil-fuel emissions of ethane and methane derived from firn air, Nature, 476, 198-201, https://doi.org/10.1038/nature10352, 2011.

Baboukas, E. D., Kanakidou, M., and Mihalopoulos, N.: Carboxylic acids in gas and particulate phase above the Atlantic Ocean, J. Geophys. Res.-Atmos., 105, 14459-14471, https://doi.org/10.1029/1999JD900977, 2000.

Bloss, C., Wagner, V., Bonzanini, A., Jenkin, M. E., Wirtz, K., Martin-Reviejo, M., and Pilling, M. J.: Evaluation of detailed aromatic mechanisms (MCMv3 and MCMv3.1) against environmental chamber data, Atmos. Chem. Phys., 5, 623-639, https://doi.org/10.5194/acp-5-623-2005, 2005.

Boersma, K. F., Eskes, H., Richter, A., De Smedt, I., Lorente, A., Beirle, S., Van Geffen, J., Peters, E., Van Roozendael, M., and Wagner, T.: QA4ECV $\mathrm{NO}_{2}$ tropospheric and stratospheric ver- tical column data from SCIAMACHY (Version 1.1), Data set, R. Netherlands Meteorol. Inst., https://doi.org/10.21944/qa4ecvno2-omi-v1.1, 2017.

Boersma, K. F., Eskes, H. J., Richter, A., De Smedt, I., Lorente, A., Beirle, S., van Geffen, J. H. G. M., Zara, M., Peters, E., Van Roozendael, M., Wagner, T., Maasakkers, J. D., van der A, R. J., Nightingale, J., De Rudder, A., Irie, H., Pinardi, G., Lambert, J.-C., and Compernolle, S. C.: Improving algorithms and uncertainty estimates for satellite $\mathrm{NO}_{2}$ retrievals: results from the quality assurance for the essential climate variables (QA4ECV) project, Atmos. Meas. Tech., 11, 6651-6678, https://doi.org/10.5194/amt-11-6651-2018, 2018.

Bouwman, A. F., Lee, D. S., Asman, W. A. H., Dentener, F. J., Van Der Hoek, K. W., and Olivier, J. G. J.: A global high-resolution emission inventory for ammonia, Global Biogeochem. Cy., 11, 561-587, https://doi.org/10.1029/97GB02266, 1997.

Bregman, B., Segers, A., Krol, M., Meijer, E., and van Velthoven, P.: On the use of mass-conserving wind fields in chemistry-transport models, Atmos. Chem. Phys., 3, 447-457, https://doi.org/10.5194/acp-3-447-2003, 2003.

Browne, E. C., Wooldridge, P. J., Min, K.-E., and Cohen, R. C.: On the role of monoterpene chemistry in the remote continental boundary layer, Atmos. Chem. Phys., 14, 1225-1238, https://doi.org/10.5194/acp-14-1225-2014, 2014.

Burkholder, J. B., Sander, S. P., Abbatt, J., Barker, J. R., Huie, R. E., Kolb, C. E., Kurylo, M. J., Orkin, V. L., Wilmouth, D. M., and Wine, P. H.: Chemical Kinetics and Photochemical Data for Use in Atmospheric Studies, Evaluation No. 18, JPL Publication 15-10, Jet Propulsion Laboratory, Pasadena, 2015.

Cai, C., Kelly, J. T., Avise, J. C., Kaduwela, A. P., and Stockwell, W. R.: Photochemical Modeling in California with Two Chemical Mechanisms: Model Intercomparison and Response to Emission Reductions, J. Air Waste Manage. Assoc., 61, 559-572, https://doi.org/10.3155/1047-3289.61.5.559, 2011.

Carter, W. P. L.: Computer modeling of environmental chamber measurements of maximum incremental reactivities of volatile organic compounds, Atmos. Environ., 29, 2513-2527, https://doi.org/10.1016/1352-2310(95)00150-W, 1995.

Carter, W. P. L.: Development of a condensed SAPRC07 chemical mechanism, Atmos. Environ., 44, 5336-5345, https://doi.org/10.1016/j.atmosenv.2010.01.024, 2010.

Christian, T. J., Kleiss, B., Yokelson, R. J., Holzinger, R., Crutzen, P. J., Hao, W. M., Saharjo, B. H., and Ward, D. E.: Comprehensive laboratory measurements of biomass-burning emissions: 1 . Emissions from Indonesian, African, and other fuels, J. Geophys. Res., 108, 4719, https://doi.org/10.1029/2003JD003704, 2003.

Crounse, J. D., Paulot, F., Kjaergaard, H. G., and Wennberg, P. O.: Peroxy radical isomerization in the oxidation of isoprene, Phys. Chem. Chem. Phys., 13, 13607, https://doi.org/10.1039/c1cp21330j, 2011.

Crutzen, P. J.: Photochemical reactions initiated by and influencing ozone in unpolluted tropospheric air, Tellus, 26, 47-57, https://doi.org/10.1111/j.2153-3490.1974.tb01951.x, 1974.

Curtis, A. R. and Sweetenham, W. P.: Facsimile/Chekmat User's Manual, Tech. rep., Oxfordshire, UK, 1987.

Dalsøren, S. B., Myhre, G., Hodnebrog, Ø., Myhre, C. L., Stohl, A., Pisso, I., Schwietzke, S., Höglund-Isaksson, L., Helmig, D., Reimann, S., Sauvage, S., Schmidbauer, N., Read, K. A., Carpenter, L. J., Lewis, A. C., Punjabi, S., Wallasch, M., Hod- 
nebrog, O., Myhre, C. L., Stohl, A., Pisso, I., Schwietzke, S., Höglund-Isaksson, L., Helmig, D., Reimann, S., Sauvage, S., Schmidbauer, N., Read, K. A., Carpenter, L. J., Lewis, A. C., Punjabi, S., and Wallasch, M.: Discrepancy between simulated and observed ethane and propane levels explained by underestimated fossil emissions, Nat. Geosci., 11, 178-184, https://doi.org/10.1038/s41561-018-0073-0, 2018.

Damian, V., Sandu, A., Damian, M., Potra, F., and Carmichael, G. R.: The kinetic preprocessor KPP-a software environment for solving chemical kinetics, Comput. Chem. Eng., 26, 1567-1579, https://doi.org/10.1016/S0098-1354(02)00128-X, 2002.

Daskalakis, N., Myriokefalitakis, S., and Kanakidou, M.: Sensitivity of tropospheric loads and lifetimes of short lived pollutants to fire emissions, Atmos. Chem. Phys., 15, 3543-3563, https://doi.org/10.5194/acp-15-3543-2015, 2015.

de Bruine, M., Krol, M., van Noije, T., Le Sager, P., and Röckmann, T.: The impact of precipitation evaporation on the atmospheric aerosol distribution in EC-Earth v3.2.0, Geosci. Model Dev., 11, 1443-1465, https://doi.org/10.5194/gmd-11-1443-2018, 2018.

Dee, D. P., Uppala, S. M., Simmons, A. J., Berrisford, P., Poli, P., Kobayashi, S., Andrae, U., Balmaseda, M. A., Balsamo, G., Bauer, P., Bechtold, P., Beljaars, A. C. M., van de Berg, I., Biblot, J., Bormann, N., Delsol, C., Dragani, R., Fuentes, M., Greer, A. J., Haimberger, L., Healy, S. B., Hersbach, H., Holm, E. V., Isaksen, L., Kallberg, P., Kohler, M., Matricardi, M., McNally, A. P., Mong-Sanz, B. M., Morcette, J.-J., Park, B.-K., Peubey, C., de Rosnay, P., Tavolato, C., Thepaut, J. N., and Vitart, F.: The ERAInterim reanalysis: Configuration and performance of the data assimilation system, Q. J. Roy. Meteorol. Soc., 137, 553-597, https://doi.org/10.1002/qj.828, 2011.

Deeter, M. N., Martínez-Alonso, S., Edwards, D. P., Emmons, L. K., Gille, J. C., Worden, H. M., Pittman, J. V., Daube, B. C., and Wofsy, S. C.: Validation of MOPITT Version 5 thermalinfrared, near-infrared, and multispectral carbon monoxide profile retrievals for 2000-2011, J. Geophys. Res.-Atmos., 118, 6710-6725, https://doi.org/10.1002/jgrd.50272, 2013.

Deeter, M. N., Edwards, D. P., Francis, G. L., Gille, J. C., Mao, D., Martínez-Alonso, S., Worden, H. M., Ziskin, D., and Andreae, M. O.: Radiance-based retrieval bias mitigation for the MOPITT instrument: the version 8 product, Atmos. Meas. Tech., 12, 45614580, https://doi.org/10.5194/amt-12-4561-2019, 2019.

Dentener, F. J. and Crutzen, P. J.: Reaction of $\mathrm{N}_{2} \mathrm{O}_{5}$ on tropospheric aerosols: Impact on the global distributions of $\mathrm{NO}_{x}, \mathrm{O}_{3}$, and $\mathrm{OH}$, J. Geophys. Res.-Atmos., 98, 7149-7163, https://doi.org/10.1029/92JD02979, 1993.

Derwent, R. G., Jenkin, M. E., and Saunders, S. M.: Photochemical ozone creation potentials for a large number of reactive hydrocarbons under European conditions, Atmos. Environ., 30, 181-199, https://doi.org/10.1016/1352-2310(95)00303-G, 1996.

Dupuy, É., Urban, J., Ricaud, P., Le Flochmoën, É., Lautié, N., Murtagh, D., De La Noë, J., El Amraoui, L., Eriksson, P., Forkman, P., Frisk, U., Jégou, F., Jiménez, C. and Olberg, M.: Stratomesospheric measurements of carbon monoxide with the Odin sub-millimetre radiometer: Retrieval and first results, Geophys. Res. Lett., 31, L20101, https://doi.org/10.1029/2004GL020558, 2004.

Ehhalt, D., Prather, M., Dentener, F., Derwent, R., Dlugokencky, E., Holland, E., Isaksen, I., Katima, J., Kirchhoff, V., Matson, P., Midgley, P. and Wang, M.: Atmospheric and Greenhouse Gases, chap. 4, in: Climate change 2001: The scientific basis. Contribution of Working Group I to the Third Assessment Report of the Intergovernmental Panel on Climate Change, edited by: Houghton, J. T., Cambridge University Press, 239-287, 2001.

Emmerson, K. M. and Evans, M. J.: Comparison of tropospheric gas-phase chemistry schemes for use within global models, Atmos. Chem. Phys., 9, 1831-1845, https://doi.org/10.5194/acp-91831-2009, 2009.

Emmons, L. K., Hauglustaine, D. A., Mfiller, J.-F., Carroll, M. A., Brasseur, G. P., Brunner, D., Staehelin, J., Thouret, V., and Marenco, A.: Data composites of airborne observations of tropospheric ozone and its precursors, 105, 20497-20538, 2000.

Emmons, L. K., Walters, S., Hess, P. G., Lamarque, J.-F., Pfister, G. G., Fillmore, D., Granier, C., Guenther, A., Kinnison, D., Laepple, T., Orlando, J., Tie, X., Tyndall, G., Wiedinmyer, C., Baughcum, S. L., and Kloster, S.: Description and evaluation of the Model for Ozone and Related chemical Tracers, version 4 (MOZART-4), Geosci. Model Dev., 3, 43-67, https://doi.org/10.5194/gmd-3-43-2010, 2010.

Evans, M. J. and Jacob, D. J.: Impact of new laboratory studies of $\mathrm{N}_{2} \mathrm{O}_{5}$ hydrolysis on global model budgets of tropospheric nitrogen oxides, ozone, and OH, Geophys. Res. Lett., 32, L09813, https://doi.org/10.1029/2005GL022469, 2005.

Eyring, V., Bony, S., Meehl, G. A., Senior, C. A., Stevens, B., Stouffer, R. J., and Taylor, K. E.: Overview of the Coupled Model Intercomparison Project Phase 6 (CMIP6) experimental design and organization, Geosci. Model Dev., 9, 1937-1958, https://doi.org/10.5194/gmd-9-1937-2016, 2016

Flemming, J., Huijnen, V., Arteta, J., Bechtold, P., Beljaars, A., Blechschmidt, A.-M., Diamantakis, M., Engelen, R. J., Gaudel, A., Inness, A., Jones, L., Josse, B., Katragkou, E., Marecal, V., Peuch, V.-H., Richter, A., Schultz, M. G., Stein, O., and Tsikerdekis, A.: Tropospheric chemistry in the Integrated Forecasting System of ECMWF, Geosci. Model Dev., 8, 975-1003, https://doi.org/10.5194/gmd-8-975-2015, 2015.

Fu, T., Jacob, D. J., Wittrock, F., Burrows, J. P., Vrekoussis, M., and Henze, D. K.: Global budgets of atmospheric glyoxal and methylglyoxal, and implications for formation of secondary organic aerosols, J. Geophys. Res., 113, D15303, https://doi.org/10.1029/2007JD009505, 2008.

Ganzeveld, L. and Lelieveld, J.: Dry deposition parameterization in a chemistry general circulation model and its influence on the distribution of reactive trace gases, J. Geophys. Res.-Atmos., 100, 20999-21012, https://doi.org/10.1029/95jd02266, 1995.

Ganzeveld, L., Lelieveld, J., and Roelofs, G. J.: A dry deposition parameterization for sulfur oxides in a chemistry and general circulation model, J. Geophys. Res.-Atmos., 103, 5679-5694, https://doi.org/10.1029/97JD03077, 1998.

Geiger, H., Barnes, I., Bejan, I., Benter, T., and Spittler, M.: The tropospheric degradation of isoprene: an updated module for the regional atmospheric chemistry mechanism, Atmos. Environ., 3, 1503-1519, https://doi.org/10.1016/S1352-2310(02)01047-6, 2003.

Gery, M. W., Whitten, G. Z., Killus, J. P., and Dodge, M. C.: A photochemical kinetics mechanism for urban and regional scale computer modeling, J. Geophys. Res., 94, 12925, https://doi.org/10.1029/JD094iD10p12925, 1989.

Goliff, W. S., Stockwell, W. R., and Lawson, C. V: The regional atmospheric chemistry mechanism, version 2, Atmos. Environ., 
68, 174-185, https://doi.org/10.1016/j.atmosenv.2012.11.038, 2013.

Granier, C. J., Lamarque, F., Mieville, A., Muller, J. F., Olivier, J., Orlando, J., Peters, J., Petron, G., Tyndall, S., and Wallens, S.: POET, a database of surface emissions of ozone precursors, GEIA-ACCENT Emiss. data portal, available at: http:// www.aero.jussieu.fr/projet/ACCENT/POET.php (last access: 10 March 2016), 2005.

Grooß, J.-U. and Russell III, J. M.: Technical note: A stratospheric climatology for $\mathrm{O}_{3}, \mathrm{H}_{2} \mathrm{O}, \mathrm{CH}_{4}, \mathrm{NO}_{x}, \mathrm{HCl}$ and $\mathrm{HF}$ derived from HALOE measurements, Atmos. Chem. Phys., 5, 2797-2807, https://doi.org/10.5194/acp-5-2797-2005, 2005.

Gros, V., Tsigaridis, K., Bonsang, B., Kanakidou, M., and Pio, C.: Factors controlling the diurnal variation of $\mathrm{CO}$ above a forested area in southeast Europe, Atmos. Environ., 36, 31273135, https://doi.org/10.1016/S1352-2310(02)00237-6, 2002.

Gross, A. and Stockwell, W. R.: Comparison of the EMEP, RADM2 and RACM mechanisms, J. Atmos. Chem., 44, 151170, https://doi.org/10.1023/A:1022483412112, 2003.

Hays, M. D., Geron, C. D., Linna, K. J., Smith, N. D., and Schauer, J. J.: Speciation of Gas-Phase and Fine Particle Emissions from Burning of Foliar Fuels, Environ. Sci. Technol., 36, 2281-2295, https://doi.org/10.1021/es0111683, 2002.

Heimann, M., Monfray, P., and Polian, G.: Long-range transport of ${ }^{222} \mathrm{Rn}$ - a test for 3D tracer models, Chem. Geol., 70, p. 90, 1988.

Henze, D. K., Hakami, A., and Seinfeld, J. H.: Development of the adjoint of GEOS-Chem, Atmos. Chem. Phys., 7, 2413-2433, https://doi.org/10.5194/acp-7-2413-2007, 2007.

Hertel, O., Berkowicz, R., Christensen, J., and Hov, Ø.: Test of two numerical schemes for use in atmospheric transportchemistry models, Atmos. Environ. Pt. A, 27, 2591-2611, https://doi.org/10.1016/0960-1686(93)90032-T, 1993.

Hodnebrog, Ø., Dalsøren, S. B., and Myhre, G.: Lifetimes, direct and indirect radiative forcing, and global warming potentials of ethane $\left(\mathrm{C}_{2} \mathrm{H}_{6}\right)$, propane $\left(\mathrm{C}_{3} \mathrm{H}_{8}\right)$, and butane $\left(\mathrm{C}_{4} \mathrm{H}_{10}\right)$, Atmos. Sci. Lett., 19, e804, https://doi.org/10.1002/asl.804, 2018.

Hoesly, R. M., Smith, S. J., Feng, L., Klimont, Z., JanssensMaenhout, G., Pitkanen, T., Seibert, J. J., Vu, L., Andres, R. J., Bolt, R. M., Bond, T. C., Dawidowski, L., Kholod, N., Kurokawa, J.-I., Li, M., Liu, L., Lu, Z., Moura, M. C. P., O'Rourke, P. R., and Zhang, Q.: Historical (1750-2014) anthropogenic emissions of reactive gases and aerosols from the Community Emissions Data System (CEDS), Geosci. Model Dev., 11, 369-408, https://doi.org/10.5194/gmd-11-369-2018, 2018.

Horowitz, L. W., Walters, S., Mauzerall, D. L., Emmons, L. K., Rasch, P. J., Granier, C., Tie, X., Lamarque, J.-F., Schultz, M. G., Tyndall, G. S., Orlando, J. J., and Brasseur, G. P.: A global simulation of tropospheric ozone and related tracers: Description and evaluation of MOZART, version 2, J. Geophys. Res.-Atmos., 108, 4784, https://doi.org/10.1029/2002JD002853, 2003.

Houweling, S., Dentener, F., and Lelieveld, J.: The impact of nonmethane hydrocarbon compounds on tropospheric photochemistry, J. Geophys. Res.-Atmos., 103, 10673-10696, https://doi.org/10.1029/97JD03582, 1998.

Hsu, J.: Diagnosing the stratosphere-to-troposphere flux of ozone in a chemistry transport model, J. Geophys. Res., 110, D19305, https://doi.org/10.1029/2005JD006045, 2005.

Huang, H.-C. and Chang, J. S.: On the performance of numerical solvers for a chemistry submodel in three-dimensional air quality models: 1. Box model simulations, J. Geophys. Res.-Atmos., 106, 20175-20188, https://doi.org/10.1029/2000JD000121, 2001.

Huijnen, V., Williams, J., van Weele, M., van Noije, T., Krol, M., Dentener, F., Segers, A., Houweling, S., Peters, W., de Laat, J., Boersma, F., Bergamaschi, P., van Velthoven, P., Le Sager, P., Eskes, H., Alkemade, F., Scheele, R., Nédélec, P., and Pätz, H.-W.: The global chemistry transport model TM5: description and evaluation of the tropospheric chemistry version 3.0, Geosci. Model Dev., 3, 445-473, https://doi.org/10.5194/gmd-3445-2010, 2010.

Huijnen, V., Pozzer, A., Arteta, J., Brasseur, G., Bouarar, I., Chabrillat, S., Christophe, Y., Doumbia, T., Flemming, J., Guth, J., Josse, B., Karydis, V. A., Marécal, V., and Pelletier, S.: Quantifying uncertainties due to chemistry modelling - evaluation of tropospheric composition simulations in the CAMS model (cycle 43R1), Geosci. Model Dev., 12, 1725-1752, https://doi.org/10.5194/gmd-12-1725-2019, 2019.

Jacob, D.: Heterogeneous chemistry and tropospheric ozone, Atmos. Environ., 34, 2131-2159, https://doi.org/10.1016/S13522310(99)00462-8, 2000.

Jacob, D. J., Field, B. D., Jin, E. M., Bey, I., Li, Q., Logan, J. A., Yantosca, R. M., and Singh, H. B.: Atmospheric budget of acetone, J. Geophys. Res.-Atmos., 107, 4100, https://doi.org/10.1029/2001JD000694, 2002.

Jégou, F., Urban, J., de La Noë, J., Ricaud, P., Le Flochmoën, E., Murtagh, D. P., Eriksson, P., Jones, A., Petelina, S., Llewellyn, E. J., Lloyd, N. D., Haley, C., Lumpe, J., Randall, C., Bevilacqua, R. M., Catoire, V., Huret, N., Berthet, G., Renard, J. B., Strong, K., Davies, J., Mc Elroy, C. T., Goutail, F., and Pommereau, J. P.: Technical Note: Validation of Odin/SMR limb observations of ozone, comparisons with OSIRIS, POAM III, ground-based and balloon-borne instruments, Atmos. Chem. Phys., 8, 3385-3409, https://doi.org/10.5194/acp-8-3385-2008, 2008.

Jenkin, M. E., Saunders, S. M., and Pilling, M. J.: The tropospheric degradation of volatile organic compounds: a protocol for mechanism development, Atmos. Environ., 31, 81-104, https://doi.org/10.1016/S1352-2310(96)00105-7, 1997.

Jenkin, M. E., Saunders, S. M., Wagner, V., and Pilling, M. J.: Protocol for the development of the Master Chemical Mechanism, MCM v3 (Part B): tropospheric degradation of aromatic volatile organic compounds, Atmos. Chem. Phys., 3, 181-193, https://doi.org/10.5194/acp-3-181-2003, 2003.

Jenkin, M. E., Young, J. C., and Rickard, A. R.: The MCM v3.3.1 degradation scheme for isoprene, Atmos. Chem. Phys., 15, 11433-11459, https://doi.org/10.5194/acp-15-11433-2015, 2015.

Kanakidou, M. and Crutzen, P. J.: The photochemical source of carbon monoxide: Importance, uncertainties and feedbacks, Chemosphere, 1, 91-109, https://doi.org/10.1016/S14659972(99)00022-7, 1999.

Kanakidou, M., Singh, H. B., Valentin, K. M., and Crutzen, P. J.: A two-dimensional study of ethane and propane oxidation in the troposphere, J. Geophys. Res., 96, 15395-15413, https://doi.org/10.1029/91jd01345, 1991.

Kim, Y., Sartelet, K., and Seigneur, C.: Comparison of two gas-phase chemical kinetic mechanisms of ozone formation over Europe, J. Atmos. Chem., 62, 89-119, https://doi.org/10.1007/s10874-009-9142-5, 2009. 
Knote, C., Tuccella, P., Curci, G., Emmons, L., Orlando, J. J., Madronich, S., Bar, R., Jim Enez-Guerrero, P., Luecken, D., Hogrefe, C., Forkel, R., Werhahn, J., Hirtl, M., Erez, J. L. P., San Jos, R., Giordano, L., Brunner, D., Yahya, K., and Zhang, Y.: Influence of the choice of gas-phase mechanism on predictions of key gaseous pollutants during the AQMEII phase-2 intercomparison, Atmos. Environ., 115, 553568, https://doi.org/10.1016/j.atmosenv.2014.11.066, 2015.

Koffi, E. N., Bergamaschi, P., Karstens, U., Krol, M., Segers, A., Schmidt, M., Levin, I., Vermeulen, A. T., Fisher, R. E., Kazan, V., Klein Baltink, H., Lowry, D., Manca, G., Meijer, H. A. J., Moncrieff, J., Pal, S., Ramonet, M., Scheeren, H. A., and Williams, A. G.: Evaluation of the boundary layer dynamics of the TM5 model over Europe, Geosci. Model Dev., 9, 3137-3160, https://doi.org/10.5194/gmd-9-3137-2016, 2016.

Krol, M., Houweling, S., Bregman, B., van den Broek, M., Segers, A., van Velthoven, P., Peters, W., Dentener, F., and Bergamaschi, P.: The two-way nested global chemistry-transport zoom model TM5: algorithm and applications, Atmos. Chem. Phys., 5, 417432, https://doi.org/10.5194/acp-5-417-2005, 2005.

Krol, M., de Bruine, M., Killaars, L., Ouwersloot, H., Pozzer, A., Yin, Y., Chevallier, F., Bousquet, P., Patra, P., Belikov, D., Maksyutov, S., Dhomse, S., Feng, W., and Chipperfield, M. P.: Age of air as a diagnostic for transport timescales in global models, Geosci. Model Dev., 11, 31093130, https://doi.org/10.5194/gmd-11-3109-2018, 2018.

Lamarque, J.-F., Bond, T. C., Eyring, V., Granier, C., Heil, A., Klimont, Z., Lee, D., Liousse, C., Mieville, A., Owen, B., Schultz, M. G., Shindell, D., Smith, S. J., Stehfest, E., Van Aardenne, J., Cooper, O. R., Kainuma, M., Mahowald, N., McConnell, J. R., Naik, V., Riahi, K., and van Vuuren, D. P.: Historical (1850-2000) gridded anthropogenic and biomass burning emissions of reactive gases and aerosols: methodology and application, Atmos. Chem. Phys., 10, 7017-7039, https://doi.org/10.5194/acp-10-7017-2010, 2010.

Lamarque, J.-F., Emmons, L. K., Hess, P. G., Kinnison, D. E., Tilmes, S., Vitt, F., Heald, C. L., Holland, E. A., Lauritzen, P. H., Neu, J., Orlando, J. J., Rasch, P. J., and Tyndall, G. K.: CAM-chem: description and evaluation of interactive atmospheric chemistry in the Community Earth System Model, Geosci. Model Dev., 5, 369-411, https://doi.org/10.5194/gmd-5369-2012, 2012.

Lana, A., Bell, T. G., Simó, R., Vallina, S. M., Ballabrera-Poy, J., Kettle, A. J., Dachs, J., Bopp, L., Saltzman, E. S., Stefels, J., Johnson, J. E., and Liss, P. S.: An updated climatology of surface dimethlysulfide concentrations and emission fluxes in the global ocean, Global Biogeochem. Cy., 25, GB1004, https://doi.org/10.1029/2010GB003850, 2011.

Landgraf, J., Crutzen, P. J., Landgraf, J., and Crutzen, P. J.: An Efficient Method for Online Calculations of Photolysis and Heating Rates, J. Atmos. Sci., 55, 863-878, https://doi.org/10.1175/15200469(1998)055<0863:AEMFOC>2.0.CO;2, 1998.

Lelieveld, J., Gromov, S., Pozzer, A., and Taraborrelli, D.: Global tropospheric hydroxyl distribution, budget and reactivity, Atmos. Chem. Phys., 16, 12477-12493, https://doi.org/10.5194/acp-1612477-2016, 2016.

Luecken, D. J., Phillips, S., Sarwar, G., and Jang, C.: Effects of using the CB05 vs. SAPRC99 vs. CB4 chemical mechanism on model predictions: Ozone and gas-phase photochemi- cal precursor concentrations, Atmos. Environ., 42, 5805-5820, https://doi.org/10.1016/j.atmosenv.2007.08.056, 2008.

Marenco, A., Thouret, V., Nédélec, P., Smit, H., Helten, M., Kley, D., Karcher, F., Simon, P., Law, K., Pyle, J., Poschmann, G., Von Wrede, R., Hume, C., and Cook, T.: Measurement of ozone and water vapor by Airbus in-service aircraft: The MOZAIC airborne program, an overview, J. Geophys. Res.-Atmos., 103, 2563125642, https://doi.org/10.1029/98JD00977, 1998.

Meijer, E. W. W., Van Velthoven, P. F. J. F. J., Brunner, D. W. W., Huntrieser, H., and Kelder, H.: Improvement and evaluation of the parameterisation of nitrogen oxide production by lightning, Phys. Chem. Earth Pt. C, 26, 577-583, https://doi.org/10.1016/S1464-1917(01)00050-2, 2001.

Metzger, S., Dentener, F., Pandis, S., and Lelieveld, J.: Gas/aerosol partitioning: 1. A computationally efficient model, J. Geophys. Res., 107, 4312, https://doi.org/10.1029/2001JD001102, 2002.

Miyazaki, K., Eskes, H., Sudo, K., Boersma, K. F., Bowman, K., and Kanaya, Y.: Decadal changes in global surface NOx emissions from multi-constituent satellite data assimilation, Atmos. Chem. Phys., 17, 807-837, https://doi.org/10.5194/acp-17-8072017, 2017.

Monks, P. S., Granier, C., Fuzzi, S., Stohl, A., Williams, M. L., Akimoto, H., Amann, M., Baklanov, A., Baltensperger, U., Bey, I., Blake, N., Blake, R. S., Carslaw, K., Cooper, O. R., Dentener, F., Fowler, D., Fragkou, E., Frost, G. J., Generoso, S., Ginoux, P., Grewe, V., Guenther, A., Hansson, H. C., Henne, S., Hjorth, J., Hofzumahaus, A., Huntrieser, H., Isaksen, I. S. A., Jenkin, M. E., Kaiser, J., Kanakidou, M., Klimont, Z., Kulmala, M., Laj, P., Lawrence, M. G., Lee, J. D., Liousse, C., Maione, M., McFiggans, G., Metzger, A., Mieville, A., Moussiopoulos, N., Orlando, J. J., O’Dowd, C. D., Palmer, P. I., Parrish, D. D., Petzold, A., Platt, U., Pöschl, U., Prévôt, A. S. H., Reeves, C. E., Reimann, S., Rudich, Y., Sellegri, K., Steinbrecher, R., Simpson, D., ten Brink, H., Theloke, J., van der Werf, G. R., Vautard, R., Vestreng, V., Vlachokostas, C., and von Glasow, R.: Atmospheric composition change - global and regional air quality, Atmos. Environ., 43, 5268-5350, https://doi.org/10.1016/j.atmosenv.2009.08.021, 2009.

Monks, S. A., Wilson, C., Emmons, L. K., Hannigan, J. W., Helmig, D., Blake, N. J., and Blake, D. R.: Using an Inverse Model to Reconcile Differences in Simulated and Observed Global Ethane Concentrations and Trends Between 2008 and 2014, J. Geophys. Res.-Atmos., 123, 11262-11282, https://doi.org/10.1029/2017JD028112, 2018.

Myriokefalitakis, S., Vrekoussis, M., Tsigaridis, K., Wittrock, F., Richter, A., Brühl, C., Volkamer, R., Burrows, J. P., and Kanakidou, M.: The influence of natural and anthropogenic secondary sources on the glyoxal global distribution, Atmos. Chem. Phys., 8, 4965-4981, https://doi.org/10.5194/acp-8-4965-2008, 2008.

Myriokefalitakis, S., Vignati, E., Tsigaridis, K., Papadimas, C., Sciare, J., Mihalopoulos, N., Facchini, M. C., Rinaldi, M., Dentener, F. J., Ceburnis, D., Hatzianastasiou, N., O’Dowd, C. D., van Weele, M., and Kanakidou, M.: Global Modeling of the Oceanic Source of Organic Aerosols, Adv. Meteorol., 2010, 939171, https://doi.org/10.1155/2010/939171, 2010.

Myriokefalitakis, S., Daskalakis, N., Gkouvousis, A., Hilboll, A., van Noije, T., Williams, J. E., Le Sager, P., Huijnen, V., Houweling, S., Bergman, T., Nüß, J. R., Vrekoussis, M., Kanakidou, M., and Krol, M. C.: TM5-MP global 
chemistry transport model (r1112) (Version r1112), Zenodo, https://doi.org/10.5281/zenodo.3952757, 2020.

Naik, V., Voulgarakis, A., Fiore, A. M., Horowitz, L. W., Lamarque, J.-F., Lin, M., Prather, M. J., Young, P. J., Bergmann, D., Cameron-Smith, P. J., Cionni, I., Collins, W. J., Dalsøren, S. B., Doherty, R., Eyring, V., Faluvegi, G., Folberth, G. A., Josse, B., Lee, Y. H., MacKenzie, I. A., Nagashima, T., van Noije, T. P. C., Plummer, D. A., Righi, M., Rumbold, S. T., Skeie, R., Shindell, D. T., Stevenson, D. S., Strode, S., Sudo, K., Szopa, S., and Zeng, G.: Preindustrial to present-day changes in tropospheric hydroxyl radical and methane lifetime from the Atmospheric Chemistry and Climate Model Intercomparison Project (ACCMIP), Atmos. Chem. Phys., 13, 5277-5298, https://doi.org/10.5194/acp13-5277-2013, 2013.

Nechita-Banda, N., Krol, M., van der Werf, G. R., Kaiser, J. W., Pandey, S., Huijnen, V., Clerbaux, C., Coheur, P., Deeter, M. N., and Röckmann, T.: Monitoring emissions from the 2015 Indonesian fires using CO satellite data, Philos. Trans. R. Soc. B Biol. Sci., 373, 20170307, https://doi.org/10.1098/rstb.2017.0307, 2018.

Nisbet, E. G., Manning, M. R., Dlugokencky, E. J., Fisher, R. E., Lowry, D., Michel, S. E., Myhre, C. L., Platt, S. M., Allen, G., Bousquet, P., Brownlow, R., Cain, M., France, J. L., Hermansen, O., Hossaini, R., Jones, A. E., Levin, I., Manning, A. C., Myhre, G., Pyle, J. A., Vaughn, B. H., Warwick, N. J., and White, J. W. C.: Very Strong Atmospheric Methane Growth in the 4 Years 2014-2017: Implications for the Paris Agreement, Global Biogeochem. Cy., 33, 318-342, https://doi.org/10.1029/2018GB006009, 2019.

Olsen, M. A.: Stratosphere-troposphere exchange of mass and ozone, J. Geophys. Res., 109, D24114, https://doi.org/10.1029/2004JD005186, 2004.

Orlando, J. J., Tyndall, G. S., and Calvert, J. G.: Thermal decomposition pathways for peroxyacetyl nitrate (PAN): Implications for atmospheric methyl nitrate levels, Atmos. Environ. Pt. A, 26, 3111-3118, https://doi.org/10.1016/0960-1686(92)90468-Z, 1992.

Paulot, F., Crounse, J. D., Kjaergaard, H. G., Kurten, A., St. Clair, J. M., Seinfeld, J. H., and Wennberg, P. O.: Unexpected Epoxide Formation in the Gas-Phase Photooxidation of Isoprene, Science, 325), 730-733, https://doi.org/10.1126/science.1172910, 2009.

Peeters, J. and Müller, J.-F.: HOx radical regeneration in isoprene oxidation via peroxy radical isomerisations. II: experimental evidence and global impact, Phys. Chem. Chem. Phys., 12, 14227, https://doi.org/10.1039/c0cp00811g, 2010.

Peeters, J., Nguyen, T. L., Vereecken, L., Peeters, J., Bayes, K. D., Ganzeveld, L., Harder, H., Lawrence, M. G., Martinez, M., Taraborrelli, D., Williams, J., Scholes, B., Steinbrecker, R., Tallamraju, R., Taylor, J. and Zimmerman, P.: HOx radical regeneration in the oxidation of isoprene, Phys. Chem. Chem. Phys., 11, 5935, https://doi.org/10.1039/b908511d, 2009.

Peeters, J., Müller, J.-F., Stavrakou, T., and Nguyen, V. S.: Hydroxyl Radical Recycling in Isoprene Oxidation Driven by Hydrogen Bonding and Hydrogen Tunneling: The Upgraded LIM1 Mechanism, J. Phys. Chem. A, 118, 8625-8643, https://doi.org/10.1021/jp5033146, 2014.

Peters, W., Krol, M. C., Dlugokencky, E. J., Dentener, F. J., Bergamaschi, P., Dutton, G., Velthoven, P. v., Miller, J. B., Bruhwiler, L., and Tans, P. P.: Toward regional-scale model- ing using the two-way nested global model TM5: Characterization of transport using SF 6, J. Geophys. Res., 109, D19314, https://doi.org/10.1029/2004JD005020, 2004.

Poisson, N., Kanakidou, M., and Crutzen, P. J.: Impact of Non-Methane Hydrocarbons on Tropospheric Chemistry and the Oxidizing Power of the Global Troposphere: 3Dimensional Modelling Results, J. Atmos. Chem., 36, 157-230, https://doi.org/10.1023/A:1006300616544, 2000.

Poisson, N., Kanakidou, M., Bonsang, B., Behmann, T., Burrows, J. P., Fischer, H., Gölz, C., Harder, H., Lewis, A., Moortgat, G. K., Nunes, T., Pio, C. A., Platt, U., Sauer, F., Schuster, G., Seakins, P., Senzig, J., Seuwen, R., Trapp, D., Volz-Thomas, A., Zenker, T., and Zitzelberger, R.: The impact of natural nonmethane hydrocarbon oxidation on the free radical and ozone budgets above a eucalyptus forest, Chemosphere, 3, 353-366, https://doi.org/10.1016/S1465-9972(01)00016-2, 2001.

Pozzer, A., Jöckel, P., Tost, H., Sander, R., Ganzeveld, L., Kerkweg, A., and Lelieveld, J.: Simulating organic species with the global atmospheric chemistry general circulation model ECHAM5/MESSy1: a comparison of model results with observations, Atmos. Chem. Phys., 7, 2527-2550, https://doi.org/10.5194/acp-7-2527-2007, 2007.

Rodigast, M., Mutzel, A., Schindelka, J., and Herrmann, H.: A new source of methylglyoxal in the aqueous phase, Atmos. Chem. Phys., 16, 2689-2702, https://doi.org/10.5194/acp-162689-2016, 2016.

Russell, G. L. and Lerner, J. A.: A New Finite-Differencing Scheme for the Tracer Transport Equation, J. Appl. Meteorol., 20, 1483-1498, https://doi.org/10.1175/15200450(1981)020<1483:ANFDSF>2.0.CO;2, 1981.

Sander, R., Kerkweg, A., Jöckel, P., and Lelieveld, J.: Technical note: The new comprehensive atmospheric chemistry module MECCA, Atmos. Chem. Phys., 5, 445-450, https://doi.org/10.5194/acp-5-445-2005, 2005.

Sander, R., Baumgaertner, A., Gromov, S., Harder, H., Jöckel, P., Kerkweg, A., Kubistin, D., Regelin, E., Riede, H., Sandu, A., Taraborrelli, D., Tost, H. and Xie, Z. Q.: The atmospheric chemistry box model CAABA/MECCA-3.0, Geosci. Model Dev., 4, 373-380, https://doi.org/10.5194/gmd-4-373-2011, 2011.

Sander, R., Baumgaertner, A., Cabrera-Perez, D., Frank, F., Gromov, S., Grooß, J.-U., Harder, H., Huijnen, V., Jöckel, P., Karydis, V. A., Niemeyer, K. E., Pozzer, A., Riede, H., Schultz, M. G., Taraborrelli, D., and Tauer, S.: The community atmospheric chemistry box model CAABA/MECCA-4.0, Geosci. Model Dev., 12, 1365-1385, https://doi.org/10.5194/gmd-121365-2019, 2019.

Sandu, A. and Sander, R.: Technical note: Simulating chemical systems in Fortran90 and Matlab with the Kinetic PreProcessor KPP-2.1, Atmos. Chem. Phys., 6, 187-195, https://doi.org/10.5194/acp-6-187-2006, 2006.

Sandu, A., Verwer, J. G., Blom, J. G., Spee, E. J., Carmichael, G. R., and Potra, F. A.: Benchmarking Stii ODE Solvers for Atmospheric Chemistry Problems II: Rosenbrock Solvers, available at: https://pdfs.semanticscholar.org/98cb/ d122e1b1054074a1a330cfc8cff36db751d9.pdf (last access: 21 August 2019), 1997.

Saunders, S. M., Jenkin, M. E., Derwent, R. G., and Pilling, M. J.: Protocol for the development of the Master Chemical Mechanism, MCM v3 (Part A): tropospheric degradation of non- 
aromatic volatile organic compounds, Atmos. Chem. Phys., 3, 161-180, https://doi.org/10.5194/acp-3-161-2003, 2003.

Saylor, R. D. and Stein, A. F.: Identifying the causes of differences in ozone production from the CB05 and CBMIV chemical mechanisms, Geosci. Model Dev., 5, 257-268, https://doi.org/10.5194/gmd-5-257-2012, 2012.

Schultz, M. G., Stadtler, S., Schröder, S., Taraborrelli, D., Franco, B., Krefting, J., Henrot, A., Ferrachat, S., Lohmann, U., Neubauer, D., Siegenthaler-Le Drian, C., Wahl, S., Kokkola, H., Kühn, T., Rast, S., Schmidt, H., Stier, P., Kinnison, D., Tyndall, G. S., Orlando, J. J., and Wespes, C.: The chemistry-climate model ECHAM6.3-HAM2.3-MOZ1.0, Geosci. Model Dev., 11, 1695-1723, https://doi.org/10.5194/gmd-11-1695-2018, 2018.

Seinfeld, J. H. and Pandis, S. N.: Atmospheric Chemistry and Physics: From Air Pollution to Climate Change, Wiley, 2006.

Shindell, D. T., Faluvegi, G., Stevenson, D. S., Krol, M. C., Emmons, L. K., Lamarque, J.-F., Pétron, G., Dentener, F. J., Ellingsen, K., Schultz, M. G., Wild, O., Amann, M., Atherton, C. S., Bergmann, D. J., Bey, I., Butler, T., Cofala, J., Collins, W. J., Derwent, R. G., Doherty, R. M., Drevet, J., Eskes, H. J., Fiore, A. M., Gauss, M., Hauglustaine, D. A., Horowitz, L. W., Isaksen, I. S. A., Lawrence, M. G., Montanaro, V., Müller, J.-F., Pitari, G., Prather, M. J., Pyle, J. A., Rast, S., Rodriguez, J. M., Sanderson, M. G., Savage, N. H., Strahan, S. E., Sudo, K., Szopa, S., Unger, N., van Noije, T. P. C., and Zeng, G.: Multimodel simulations of carbon monoxide: Comparison with observations and projected near-future changes, J. Geophys. Res., 111, D19306, https://doi.org/10.1029/2006JD007100, 2006.

Silvern, R. F., Jacob, D. J., Travis, K. R., Sherwen, T., Evans, M. J., Cohen, R. C., Laughner, J. L., Hall, S. R., Ullmann, K., Crounse, J. D., Wennberg, P. O., Peischl, J., and Pollack, I. B.: Observed $\mathrm{NO} / \mathrm{NO}_{2}$ Ratios in the Upper Troposphere Imply Errors in $\mathrm{NO}-\mathrm{NO}_{2}-\mathrm{O}_{3}$ Cycling Kinetics or an Unaccounted NOx Reservoir, Geophys. Res. Lett., 45, 4466-4474, https://doi.org/10.1029/2018GL077728, 2018.

Sindelarova, K., Granier, C., Bouarar, I., Guenther, A., Tilmes, S., Stavrakou, T., Müller, J.-F., Kuhn, U., Stefani, P., and Knorr, W.: Global data set of biogenic VOC emissions calculated by the MEGAN model over the last 30 years, Atmos. Chem. Phys., 14, 9317-9341, https://doi.org/10.5194/acp-14-9317-2014, 2014.

Spiro, P. A., Jacob, D. J., and Logan, J. A.: Global inventory of sulfur emissions with $1^{\circ} \times 1^{\circ}$ resolution, J. Geophys. Res., 97, 6023, https://doi.org/10.1029/91JD03139, 1992.

Spivakovsky, C. M., Logan, J. A., Montzka, S. A., Balkanski, Y. J., Foreman-Fowler, M., Jones, D. B. A., Horowitz, L. W., Fusco, A. C., Brenninkmeijer, C. A. M., Prather, M. J., Wofsy, S. C., and McElroy, M. B.: Three-dimensional climatological distribution of tropospheric $\mathrm{OH}$ : Update and evaluation, J. Geophys. Res.-Atmos., 105, 8931-8980, https://doi.org/10.1029/1999JD901006, 2000.

Stavrakou, T., Müller, J.-F., De Smedt, I., Van Roozendael, M., van der Werf, G. R., Giglio, L., and Guenther, A.: Evaluating the performance of pyrogenic and biogenic emission inventories against one decade of space-based formaldehyde columns, Atmos. Chem. Phys., 9, 1037-1060, https://doi.org/10.5194/acp-91037-2009, 2009a.

Stavrakou, T., Müller, J.-F., De Smedt, I., Van Roozendael, M., Kanakidou, M., Vrekoussis, M., Wittrock, F., Richter, A., and Burrows, J. P.: The continental source of glyoxal es- timated by the synergistic use of spaceborne measurements and inverse modelling, Atmos. Chem. Phys., 9, 8431-8446, https://doi.org/10.5194/acp-9-8431-2009, 2009b.

Stevenson, D. S., Dentener, F. J., Schultz, M. G., Ellingsen, K., van Noije, T. P. C., Wild, O., Zeng, G., Amann, M., Atherton, C. S., Bell, N., Bergmann, D. J., Bey, I., Butler, T., Cofala, J., Collins, W. J., Derwent, R. G., Doherty, R. M., Drevet, J., Eskes, H. J., Fiore, A. M., Gauss, M., Hauglustaine, D. A., Horowitz, L. W., Isaksen, I. S. A., Krol, M. C., Lamarque, J.-F., Lawrence, M. G., Montanaro, V., Müller, J.-F., Pitari, G., Prather, M. J., Pyle, J. A., Rast, S., Rodriguez, J. M., Sanderson, M. G., Savage, N. H., Shindell, D. T., Strahan, S. E., Sudo, K., and Szopa, S.: Multimodel ensemble simulations of present-day and near-future tropospheric ozone, J. Geophys. Res., 111, D08301, https://doi.org/10.1029/2005JD006338, 2006.

Stockwell, W. R., Kirchner, F., Kuhn, M., and Seefeld, S.: A new mechanism for regional atmospheric chemistry modeling, J. Geophys. Res.-Atmos., 102, 25847-25879, https://doi.org/10.1029/97JD00849, 1997.

Thouret, V., Marenco, A., Logan, J. A., Nédélec, P., and Grouhel, C.: Comparisons of ozone measurements from the MOZAIC airborne program and the ozone sounding network at eight locations, J. Geophys. Res.-Atmos., 103, 25695-25720, https://doi.org/10.1029/98JD02243, 1998.

Tiedtke, M.: A Comprehensive Mass Flux Scheme for $\mathrm{Cu}$ mulus Parameterization in Large-Scale Models, Mon. Weather Rev., 117, 1779-1800, https://doi.org/10.1175/15200493(1989)117<1779:ACMFSF>2.0.CO;2, 1989.

Toon, G. C., Blavier, J.-F. L., and Sung, K.: Measurements of atmospheric ethene by solar absorption FTIR spectrometry, Atmos. Chem. Phys., 18, 5075-5088, https://doi.org/10.5194/acp18-5075-2018, 2018.

Travis, K. R., Jacob, D. J., Fisher, J. A., Kim, P. S., Marais, E. A., Zhu, L., Yu, K., Miller, C. C., Yantosca, R. M., Sulprizio, M. P., Thompson, A. M., Wennberg, P. O., Crounse, J. D., St. Clair, J. M., Cohen, R. C., Laughner, J. L., Dibb, J. E., Hall, S. R., Ullmann, K., Wolfe, G. M., Pollack, I. B., Peischl, J., Neuman, J. A., and Zhou, X.: Why do models overestimate surface ozone in the Southeast United States?, Atmos. Chem. Phys., 16, 1356113577, https://doi.org/10.5194/acp-16-13561-2016, 2016.

Tsigaridis, K. and Kanakidou, M.: Importance of volatile organic compounds photochemistry over a forested area in central Greece, Atmos. Environ., 36, 3137-3146, https://doi.org/10.1016/S1352-2310(02)00234-0, 2002.

Tsigaridis, K. and Kanakidou, M.: Global modelling of secondary organic aerosol in the troposphere: a sensitivity analysis, Atmos. Chem. Phys., 3, 1849-1869, https://doi.org/10.5194/acp-3-18492003, 2003.

Urban, J., Pommier, M., Murtagh, D. P., Santee, M. L., and Orsolini, Y. J.: Nitric acid in the stratosphere based on Odin observations from 2001 to 2009 - Part 1: A global climatology, Atmos. Chem. Phys., 9, 7031-7044, https://doi.org/10.5194/acp-9-70312009, 2009.

van Marle, M. J. E., Kloster, S., Magi, B. I., Marlon, J. R., Daniau, A.-L., Field, R. D., Arneth, A., Forrest, M., Hantson, S., Kehrwald, N. M., Knorr, W., Lasslop, G., Li, F., Mangeon, S., Yue, C., Kaiser, J. W., and van der Werf, G. R.: Historic global biomass burning emissions for CMIP6 (BB4CMIP) based on merging satellite observations with proxies and fire 
models (1750-2015), Geosci. Model Dev., 10, 3329-3357, https://doi.org/10.5194/gmd-10-3329-2017, 2017.

van Noije, T. P. C., Eskes, H. J., van Weele, M., and van Velthoven, P. F. J.: Implications of the enhanced Brewer-Dobson circulation in European Centre for Medium-Range Weather Forecasts reanalysis ERA-40 for the stratosphere-troposphere exchange of ozone in global chemistry transport models, J. Geophys. Res., 109, D19308, https://doi.org/10.1029/2004JD004586, 2004.

van Noije, T. P. C., Le Sager, P., Segers, A. J., van Velthoven, P. F. J., Krol, M. C., Hazeleger, W., Williams, A. G., and Chambers, S. D.: Simulation of tropospheric chemistry and aerosols with the climate model EC-Earth, Geosci. Model Dev., 7, 2435-2475, https://doi.org/10.5194/gmd-7-2435-2014, 2014.

van Noije, T.: EC-Earth3-AerChem, A global climate model with interactive aerosols and atmospheric chemistry for use in CMIP6, in preparation, 2020.

Vignati, E., Wilson, J., and Stier, P.: M7: An efficient sizeresolved aerosol microphysics module for large-scale aerosol transport models, J. Geophys. Res.-Atmos., 109, D22202, https://doi.org/10.1029/2003JD004485, 2004.

Voulgarakis, A., Naik, V., Lamarque, J.-F., Shindell, D. T., Young, P. J., Prather, M. J., Wild, O., Field, R. D., Bergmann, D., CameronSmith, P., Cionni, I., Collins, W. J., Dalsøren, S. B., Doherty, R. M., Eyring, V., Faluvegi, G., Folberth, G. A., Horowitz, L. W., Josse, B., MacKenzie, I. A., Nagashima, T., Plummer, D. A., Righi, M., Rumbold, S. T., Stevenson, D. S., Strode, S. A., Sudo, K., Szopa, S., and Zeng, G.: Analysis of present day and future $\mathrm{OH}$ and methane lifetime in the ACCMIP simulations, Atmos. Chem. Phys., 13, 2563-2587, https://doi.org/10.5194/acp13-2563-2013, 2013.

Vrekoussis, M., Liakakou, E., Mihalopoulos, N., Kanakidou, M., Crutzen, P. J., and Lelieveld, J.: Formation of $\mathrm{HNO}_{3}$ and $\mathrm{NO}_{3}$ - in the anthropogenically-influenced eastern Mediterranean marine boundary layer, Geophys. Res. Lett., 33, L05811, https://doi.org/10.1029/2005GL025069, 2006.

Vrekoussis, M., Wittrock, F., Richter, A., and Burrows, J. P.: Temporal and spatial variability of glyoxal as observed from space, Atmos. Chem. Phys., 9, 4485-4504, https://doi.org/10.5194/acp9-4485-2009, 2009.

Wallington, T. J., Ammann, M., Cox, R. A., Crowley, J. N., Herrmann, H., Jenkin, M. E., McNeill, V., Mellouki, A., Rossi, M. J., and Troe, J.: IUPAC Task group on atmospheric chemical kinetic data evaluation: Evaluated kinetic data, available at: http://iupac.pole-ether.fr (last access: 10 April 2019), 2018.

Ware, G. W.: Methyl ethyl ketone, in: Reviews of Environmental Contamination and Toxicology, 165-174, 1988.

Wesely, M. L.: Parameterization of surface resistances to gaseous dry deposition in regional-scale numerical models, Atmos. Environ., 41, 52-63, https://doi.org/10.1016/j.atmosenv.2007.10.058, 1989.
Wild, O.: Modelling the global tropospheric ozone budget: exploring the variability in current models, Atmos. Chem. Phys., 7, 2643-2660, https://doi.org/10.5194/acp-7-2643-2007, 2007.

Williams, J. E., Strunk, A., Huijnen, V., and van Weele, M.: The application of the Modified Band Approach for the calculation of on-line photodissociation rate constants in TM5: implications for oxidative capacity, Geosci. Model Dev., 5, 15-35, https://doi.org/10.5194/gmd-5-15-2012, 2012.

Williams, J. E., van Velthoven, P. F. J., and Brenninkmeijer, C. A. M.: Quantifying the uncertainty in simulating global tropospheric composition due to the variability in global emission estimates of Biogenic Volatile Organic Compounds, Atmos. Chem. Phys., 13, 2857-2891, https://doi.org/10.5194/acp13-2857-2013, 2013.

Williams, J. E., Boersma, K. F., Le Sager, P., and Verstraeten, W. W.: The high-resolution version of TM5-MP for optimized satellite retrievals: description and validation, Geosci. Model Dev., 10, 721-750, https://doi.org/10.5194/gmd-10-721-2017, 2017.

Yarwood, G., Rao, S., and Yocke, M.: Updates to the carbon bond chemical mechanism: CB05 - Prepared for Deborah Luecken U.S. Environmental Protection Agency Research Triangle Park, NC 27703, available at: http://www.camx.com/files/cb05_fina_ report_120805.aspx (last access: 16 March 2017), 2005.

Yienger, J. J. and Levy, H.: Empirical model of global soilbiogenic NO $\chi$ emissions, J. Geophys. Res., 100, 11447, https://doi.org/10.1029/95JD00370, 1995.

Young, A. H., Keene, W. C., Pszenny, A. A. P., Sander, R., Thornton, J. A., Riedel, T. P., and Maben, J. R.: Phase partitioning of soluble trace gases with size-resolved aerosols in near-surface continental air over northern Colorado, USA, during winter, J. Geophys. Res.-Atmos., 118, 9414-9427, https://doi.org/10.1002/jgrd.50655, 2013.

Zaveri, R. A. and Peters, L. K.: A new lumped structure photochemical mechanism for large-scale applications, J. Geophys. Res.-Atmos., 104, 30387-30415, https://doi.org/10.1029/1999JD900876, 1999.

Zimmermann, P. H.: A handy global tracer model, in Air Pollution Modeling and its Applications VI, in Plenum, edited by H. van Dop, NATO/CCMS, New York, 593-608, 1988.

Ziskin, D.: Measurements Of Pollution In The Troposphere (MOPITT) Level 2 Derived CO (Near and Thermal Infrared Radiances) (MOP02J) V008 Beta, Data set, Atmospheric Sci. Data Center, NASA, https://doi.org/10.5067/TERRA/MOPITT/MOP02J_L2.008, 2019. 